\title{
Existence and Stability of Implicit Fractional Differential Equations with Stieltjes Boundary Conditions Involving Hadamard Derivatives
}

\author{
Danfeng Luo $\mathbb{D}^{1},{ }^{1}$ Mehboob Alam, ${ }^{2}$ Akbar Zada ${ }^{D},{ }^{2}$ Usman Riaz, ${ }^{2}$ and Zhiguo Luo $\mathbb{D}^{3}$ \\ ${ }^{1}$ Department of Mathematics, Guizhou University, Guiyang 550025, China \\ ${ }^{2}$ Department of Mathematics, University of Peshawar, Peshawar, Khyber Pakhtunkhwa, Pakistan \\ ${ }^{3}$ Key Laboratory of Computing and Stochastic Mathematics (Ministry of Education), School of Mathematics and Statistics, \\ Hunan Normal University, Changsha 410081, China \\ Correspondence should be addressed to Akbar Zada; akbarzada@uop.edu.pk
}

Received 4 September 2020; Revised 7 November 2020; Accepted 22 February 2021; Published 23 March 2021

Academic Editor: Peter Giesl

Copyright (C) 2021 Danfeng Luo et al. This is an open access article distributed under the Creative Commons Attribution License, which permits unrestricted use, distribution, and reproduction in any medium, provided the original work is properly cited.

In this article, we make analysis of the implicit fractional differential equations involving integral boundary conditions associated with Stieltjes integral and its corresponding coupled system. We use some sufficient conditions to achieve the existence and uniqueness results for the given problems by applying the Banach contraction principle, Schaefer's fixed point theorem, and Leray-Schauder result of the cone type. Moreover, we present different kinds of stability such as Hyers-Ulam stability, generalized Hyers-Ulam stability, Hyers-Ulam-Rassias stability, and generalized Hyers-Ulam-Rassias stability by using the classical technique of functional analysis. At the end, the results are verified with the help of examples.

\section{Introduction}

Fractional-order derivatives are the generalization of integer-order derivatives. The idea of fractional-order derivatives has been introduced at the end of the sixteen century when Leibniz used the notation $\left(d^{\alpha} / d \sigma^{\alpha}\right)$ for $\alpha^{\text {th }}$-order derivative. It is well known that if $\phi(\sigma)=(1 / 2) \sigma^{2}$, then $\phi^{\prime}(\sigma)=\sigma$ and $\phi^{\prime \prime}(\sigma)=1$, respectively. But, if the order is in the fractional form, i.e., $\alpha=(1 / 2), \alpha=(1 / 3)$, and so on, then what would be the result? This question was mentioned in a letter to L'Hospital by Leibniz in (1695) [1]. Since then, several mathematicians such as Fourier, Laplace, and Letnikov contributed to the development of the fractional calculus. Riemann and Liouville had worked on this problem and introduced the Riemann-Liouville fractional derivative, which was further generalized by Caputo and can be considered as the fundamental concept in fractional calculus. Fractional calculus has many applications in many scientific disciplines, e.g., in the fields of signal and image processing [2], mathematical biological systems [3], electronics, economics, control theory [4], chemistry, biophysics, and blood flow phenomena. For more applications of fractional differential equations, we refer the reader to $[5,6]$ and the references cited therein.

In 1892 [7], Hadamard presented a concept of fractional derivative, which is different from Caputo- and Riemann-Liouville-type fractional derivatives. An important feature of the Hadamard fractional derivative is that it contains a logarithmic function of an arbitrary exponent in its definition. Here, we stress that the studies about Hadamard fractional differential equations are still at the early stage and need additional analysis. For more details and recent contributions to the topic, see [8-11].

In the field of fractional differential equations, the area which received great attention from researchers is the existence of solutions of different boundary-value problems. Many researchers used different fixed-point theorems and developed different approaches for the existence of solutions of complicated boundary-value problems. For details, we refer the reader to [12-14]. The study of coupled systems of 
differential equations is also very significant because these types of systems appear naturally in many problems of applied nature. For details and examples, the reader may see [15-18] and the references cited therein.

Another area of research which has received great attention from researchers in the field of fractional differential equations is the notion of stability in the sense of Ulam. There are different kinds of Ulam's stabilities, i.e., Hyers-Ulam stability $(\mathscr{H} \mathscr{U} \mathcal{S})$, generalized Hyers-Ulam stability $(\mathscr{G} \mathscr{H} \mathscr{U} \mathcal{S})$, Hyers-Ulam-Rassias stability $(\mathscr{H} \mathscr{U} \mathscr{R} \mathcal{S})$, and generalized Hyers-Ulam-Rassias stability $(\mathscr{G} \mathscr{H} \mathscr{R} \mathcal{S})$. The Ulam-type stability was first introduced by Ulam [19] in 1940 and then was studied and generalized by many mathematicians with different approaches [10, 20-29].

Ren and Zhai [30] discussed the existence of a unique solution and multiple positive solutions with nonlocal boundary conditions for the system involving standard Riemann-Liouville fractional $q$-derivatives:

$$
\left\{\begin{array}{l}
\mathfrak{D}_{q}^{\alpha} \mathrm{u}(\sigma)+\phi(\sigma, \mathrm{u}(\sigma))=0, \quad \sigma \in J, \\
\left.\mathrm{u}(\sigma)\right|_{\sigma=0}=\left.\mathfrak{D}_{q}^{\alpha-2} \mathrm{u}(\sigma)\right|_{\sigma=0}=0, \\
\left.\mathfrak{D}_{q}^{\alpha-1} \mathrm{u}(\sigma)\right|_{\sigma=1}=\alpha[\mathrm{u}]+\int_{0}^{\zeta} \varphi(\sigma) \mathfrak{D}_{q}^{\beta} \mathrm{u}(\sigma) d_{q} \sigma,
\end{array}\right.
$$

where $J=[0,1]$ and $\mathfrak{D}_{q}^{\alpha}$ denote the standard Riemann-Liouville fractional $\mathrm{q}$-derivatives of order $\alpha$, with $\alpha \in(2,3], \varphi \in L^{1}[0,1]$ being nonnegative.

Benchohra and Lazreg [8] studied the existence and Hyers-Ulam stability of the following implicit fractional differential equations $\left(\mathscr{F} \mathscr{D} \mathscr{E}^{\prime} s\right)$ involving Hadamard derivatives:

$$
\left\{\begin{array}{l}
{ }_{H} \mathfrak{D}^{\alpha} \mathrm{u}(\sigma)=\phi_{1}\left(\sigma, \mathrm{u}(\sigma), \mathfrak{D}^{\alpha} \mathrm{u}(\sigma)\right), \quad \alpha \in(0,1), \\
\mathrm{u}(1)=\mathrm{u}_{1}, \quad \mathrm{u}_{1} \in \mathbb{R},
\end{array}\right.
$$

where $\sigma \in[1, \mathscr{T}], \mathscr{T}>1$ and ${ }_{H} \mathfrak{D}^{\alpha}$ denotes the Hadamard fractional derivative of order $\alpha$.

Riaz et al. [10] studied the existence, uniqueness, and stability of the solution of a nonlinear coupled system of impulsive $\mathscr{F} \mathscr{D} \mathscr{E} s$ using Hadamard derivatives of the following form:

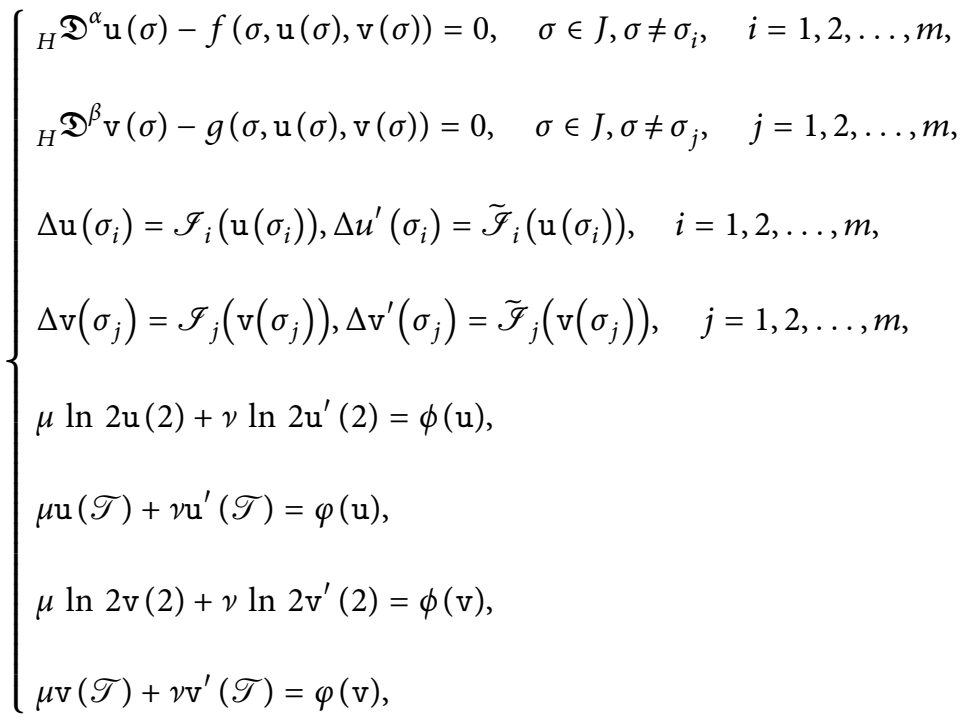


where $1<\alpha, \beta \leq 2, f, g: J \times \mathbb{R}^{2} \longrightarrow \mathbb{R}$ and $\phi, \varphi: \mathscr{C}(J, \mathbb{R}) \longrightarrow \mathbb{R}$ are continuous functions.
Riaz et al. [31] studied the existence, uniqueness, and stability of solution of coupled implicit impulsive fractional differential equations using Hadamard derivatives:

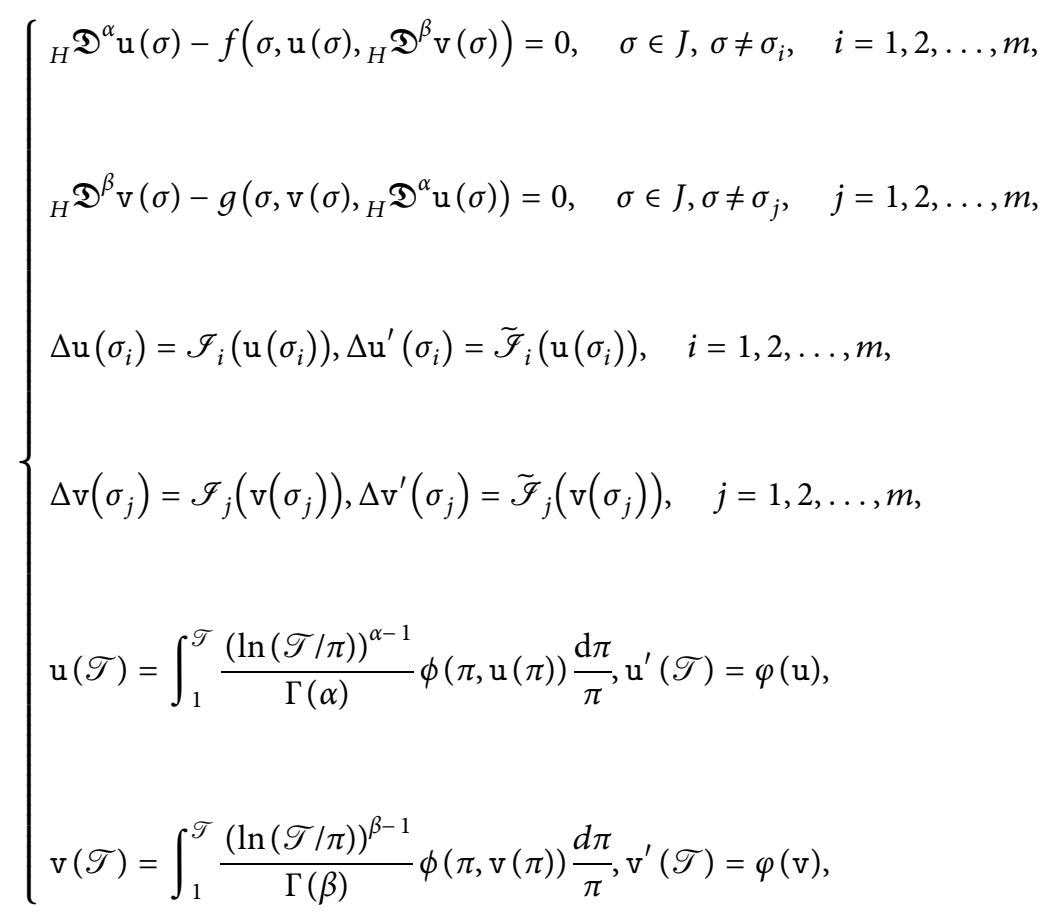

where $\quad 1<\alpha, \beta \leq 2, f, g: J \times \mathbb{R}^{2} \longrightarrow \mathbb{R} \quad$ and $\quad$ Stieltjes integral condition instead of the Riemann-Liouville $\phi, \varphi: \mathscr{C}(J, \mathbb{R}) \longrightarrow \mathbb{R}$ are continuous functions. fractional q-derivative and present its existence, uniqueness,

In this article, we extend the system of Ren and Zhai [30] at least one solution, and different kinds of Ulam's stabilities. to implicit Hadamard fractional derivatives having the We study the following system:

$$
\left\{\begin{array}{l}
{ }_{H} \mathfrak{D}^{\alpha} \mathrm{u}(\sigma)+\phi\left(\sigma, \mathrm{u}(\sigma),{ }_{H} \mathfrak{D}^{\alpha} \mathrm{u}(\sigma)\right)=0, \quad \sigma \in J, \\
\left.\mathrm{u}(\sigma)\right|_{\sigma=1}=\left.{ }_{H} \mathfrak{D}^{\alpha-2} \mathrm{u}(\sigma)\right|_{\sigma=1}=0, \\
\left.{ }_{H} \mathfrak{D}^{\alpha-1} \mathrm{u}(\sigma)\right|_{\sigma=2}=\alpha[\mathrm{u}]+\int_{1}^{\zeta} \varphi(\sigma)_{H} \mathfrak{D}^{\beta} \mathrm{u}(\sigma) \mathrm{d} \sigma,
\end{array}\right.
$$

where $\alpha \in(2,3], \phi: J=[1,2] \times \mathbb{R} \times \mathbb{R} \longrightarrow \mathbb{R}$ is the continuous function and ${ }_{H} \mathfrak{D}^{\alpha},{ }_{H} \mathfrak{D}^{\beta}$ are the Hadamard fractional derivatives of order $\alpha$, with $\alpha-1-\beta>0, \varphi \in L^{1}[1,2]$ being non-negative, and $\alpha[\mathrm{u}]$ is a linear function given by

$$
\alpha[\mathrm{u}]=\int_{1}^{2} \mathrm{u}(\sigma) \mathrm{d} \mu(\sigma)
$$

involving the Stieltjes integral with respect to the function $\mu:[1,2] \longrightarrow \mathbb{R}, \mu(\sigma)$ is right continuous on $[1,2)$, left continuous at $\sigma=2$. Particularly, $\mu$ is a nondecreasing function with $\mu(1)=0$; then, $\mathrm{d} \mu$ is a positive Stieltjes measure.

We also extend system (5) to the coupled system and discuss its existence, uniqueness, at least one solution, and different kinds of Ulam's stabilities of the problem: 


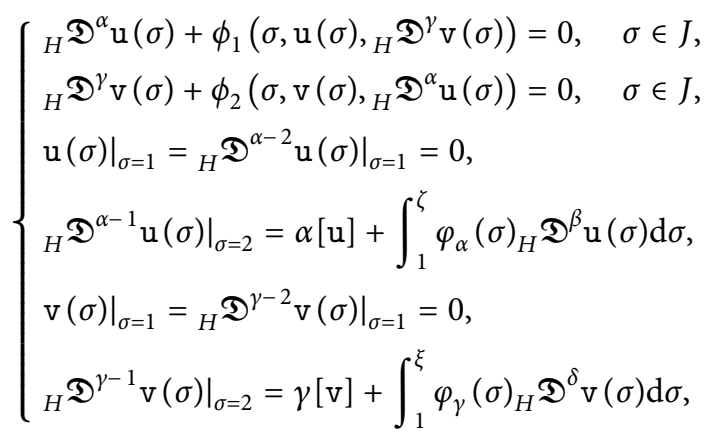

where $\alpha, \gamma \in(2,3], \phi_{1}, \phi_{2}: J=[1,2] \times \mathbb{R} \times \mathbb{R} \longrightarrow \mathbb{R}$ are the continuous functions and $\mathfrak{D}^{\alpha},{ }_{H} \mathfrak{D}^{\beta},{ }_{H} \mathfrak{D}^{\gamma}$, and ${ }_{H} \mathfrak{D}^{\delta}$ are the Hadamard fractional derivatives of orders $\alpha, \gamma$, respectively with $\quad \alpha-1-\beta>0, \gamma-1-\delta>0, \varphi_{\alpha}, \varphi_{\gamma} \in L^{1}[1,2] \quad$ being nonnegative, and $\alpha[\mathrm{u}], \gamma[\mathrm{v}]$ are linear functions given by

$$
\begin{aligned}
& \alpha[\mathrm{u}]=\int_{1}^{2} \mathrm{u}(\sigma) \mathrm{d} \mu(\sigma), \\
& \gamma[\mathrm{v}]=\int_{1}^{2} \mathrm{v}(\sigma) \mathrm{d} \mu(\sigma),
\end{aligned}
$$

involving the Stieltjes integral with respect to the function $\mu:[1,2] \longrightarrow \mathbb{R}, \mu(\sigma)$ is right continuous on $[1,2)$, left continuous at $\sigma=2$. Particularly, $\mu$ is a nondecreasing function with $\mu(1)=0$; then, $\mathrm{d} \mu$ is a positive Stieltjes measure.

The rest of the article is arranged as follows: In Section 2, we present some basic definitions, lemmas, and theorems that are used in our main results. In Section 3, we use different conditions and some standard fixed-point theorems for the existence and uniqueness of solutions to the given system (5) and its corresponding coupled system (7). In Section 4, we present Ulam's stabilities for the given systems (5) and (7) under some specific conditions. At the end, examples are given to illustrate the main results.

Throughout the paper, we assume that

$\left(\mathbf{M}_{1}\right): \Delta=\Gamma(\alpha-\beta)-\int_{1}^{\zeta} \varphi(\sigma)(\ln \sigma)^{\alpha-1-\beta} \mathrm{d} \sigma-\Gamma(\alpha-\beta)$ $/ \Gamma(\alpha) \int_{1}^{2}(\ln \sigma)^{\alpha-1} \mathrm{~d} \mu(\sigma)$.

$\left(\mathbf{M}_{2}\right): \phi: J \times \mathbb{R} \times \mathbb{R} \longrightarrow \mathbb{R}$ is continuous.

$\left(\mathbf{M}_{3}\right):$ for $\sigma \in J$ and $\mathrm{u}, \mathrm{z} \in \mathbb{X}$, there are $\pi_{1}, \pi_{2}, \pi_{3} \in C\left(J, \mathbb{R}^{+}\right)$such that

$|\phi(\sigma, \mathrm{u}(\sigma), \mathbf{z}(\sigma))| \leq \pi_{1}(\sigma)+\pi_{2}(\sigma)|\mathrm{u}(\sigma)|+\pi_{3}(\sigma)|\mathrm{z}(\sigma)|$,

with $\quad \pi_{1}^{*}=\sup _{\sigma \in J} \pi_{1}(\sigma), \pi_{2}^{*}=\sup _{\sigma \in J} \pi_{2}(\sigma), \quad$ and $\pi_{3}^{*}=\sup _{\sigma \in J} \pi_{3}(\sigma)<1$.

$\left(\mathbf{M}_{4}\right)$ : for all $\mathrm{u}, \mathrm{z}, \widehat{\mathrm{u}}, \widehat{\mathbf{z}} \in \mathbb{R}$ and for each $\sigma \in J$, there exist constants $\mathfrak{Q}_{\phi}>0,0<\mathfrak{Q}_{\phi}^{*}<1$ such that

$$
|\phi(\sigma, u, z)-\phi(\sigma, \widehat{u}, \widehat{z})| \leq \mathfrak{Q}_{\phi}|u-\widehat{u}|+\mathfrak{Q}_{\phi}^{*}|z-\widehat{z}| .
$$

$\left(\mathbf{M}_{5}\right)$ : for $\sigma \in J$, there exists a constant $\mathfrak{E}$ such that

$$
|\varphi(\sigma)| \leq \mathfrak{F} .
$$

$\left(\mathbf{M}_{6}\right)$ : let $\Psi \in C(J, \mathbb{R})$ be an increasing function; then, there is $\Omega_{\Psi}>0$ such that, for each $\sigma \in J$, the inequality

$$
{ }_{H} \mathfrak{J}^{\alpha} \Psi(\sigma) \leq \Omega_{\Psi} \Psi(\sigma),
$$

holds.

$$
\begin{gathered}
\left(\mathbf{M}_{7}\right): \Delta_{\alpha}=\Gamma(\alpha-\beta)-\int_{1}^{\zeta} \varphi_{\alpha}(\sigma)(\ln \sigma)^{\alpha-1-\beta} \mathrm{d} \sigma-\Gamma(\alpha- \\
\beta) / \Gamma(\alpha) \int_{1}^{2}(\ln \sigma)^{\alpha-1} \mathrm{~d} \mu(\sigma), \text { and } \\
\Delta_{\gamma}=\Gamma(\gamma-\xi)-\int_{1}^{\xi} \varphi_{\gamma}(\sigma)(\ln \sigma)^{\gamma-1-\xi} \mathrm{d} \sigma \\
-\frac{\Gamma(\gamma-\xi)}{\Gamma(\gamma)} \int_{1}^{2}(\ln \sigma)^{\gamma-1} \mathrm{~d} \mu(\sigma) .
\end{gathered}
$$

$\left(\mathbf{M}_{8}\right): \phi_{1}, \phi_{2}: J \times \mathbb{R} \times \mathbb{R} \longrightarrow \mathbb{R}$ are continuous.

$\left(\mathbf{M}_{9}\right):$ for $\sigma \in J$ and $\mathrm{u}, \mathrm{z} \in \mathbb{X}$, there are $\pi_{4}, \pi_{5}, \pi_{6} \in C\left(J, \mathbb{R}^{+}\right)$such that

$\left|\phi_{1}(\sigma, \mathrm{u}(\sigma), \mathrm{z}(\sigma))\right| \leq \pi_{4}(\sigma)+\pi_{5}(\sigma)|\mathrm{u}(\sigma)|+\pi_{6}(\sigma)|\mathrm{z}(\sigma)|$,

with $\quad \pi_{4}^{*}=\sup _{\sigma \in J} \pi_{4}(\sigma), \pi_{5}^{*}=\sup _{\sigma \in J} \pi_{5}(\sigma), \quad$ and $\pi_{6}^{*}=\sup _{\sigma \in J} \pi_{6}(\sigma)<1$.

Similarly, for $\sigma \in J$ and $\mathrm{v}, \mathrm{y} \in \mathbb{X}$, there are $\pi_{7}, \pi_{8}, \pi_{9} \in C\left(J, \mathbb{R}^{+}\right)$such that

$\left|\phi_{2}(\sigma, \mathrm{v}(\sigma), \mathrm{y}(\sigma))\right| \leq \pi_{7}(\sigma)+\pi_{8}(\sigma)|\mathrm{v}(\sigma)|+\pi_{9}(\sigma)|\mathrm{y}(\sigma)|$,

with $\quad \pi_{7}^{*}=\sup _{\sigma \in J} \pi_{7}(\sigma), \pi_{8}^{*}=\sup _{\sigma \in J} \pi_{8}(\sigma), \quad$ and $\pi_{9}^{*}=\sup _{\sigma \in J} \pi_{9}(\sigma)<1$.

$\left(\mathbf{M}_{10}\right)$ : for all $\mathrm{u}, \mathrm{z}, \widehat{\mathrm{u}}, \widehat{\mathrm{z}}, \mathrm{v}, \mathrm{y}, \widehat{\mathrm{v}}, \widehat{\mathrm{y}} \in \mathbb{R}$ and for each $\sigma \in J$, there exist constants $\mathfrak{Q}_{\phi_{1}}, \mathfrak{Q}_{\phi_{2}}>0,0<\mathfrak{Q}_{\phi_{1}}^{*}, \mathfrak{Q}_{\phi_{2}}^{*}<1$ such that

$$
\begin{aligned}
& \left|\phi_{1}(\sigma, \mathrm{u}, \mathrm{z})-\phi_{1}(\sigma, \widehat{\mathrm{u}}, \widehat{\mathrm{z}})\right| \leq \mathfrak{\Omega}_{\phi_{1}}|\mathrm{u}-\widehat{\mathrm{u}}|+\mathfrak{Q}_{\phi_{1}}^{*}|\mathrm{z}-\widehat{\mathrm{z}}| \\
& \left|\phi_{2}(\sigma, \mathrm{v}, \mathrm{y})-\phi_{2}(\sigma, \widehat{\mathrm{v}}, \widehat{\mathrm{y}})\right| \leq \mathfrak{\Omega}_{\phi_{2}}|\mathrm{v}-\widehat{\mathrm{v}}|+\mathfrak{Q}_{\phi_{2}}^{*}|\mathrm{y}-\widehat{\mathrm{y}}| .
\end{aligned}
$$

$\left(\mathbf{M}_{11}\right):$ for $\sigma \in J$, there exist constants $\mathfrak{F}_{\alpha}, \mathfrak{E}_{\gamma}$ such that

$$
\begin{aligned}
& \left|\varphi_{\alpha}(\sigma)\right| \leq \mathfrak{F}_{\alpha}, \\
& \left|\varphi_{\gamma}(\sigma)\right| \leq \mathfrak{F}_{\gamma} .
\end{aligned}
$$

$\left(\mathbf{M}_{12}\right)$ : let $\Psi_{\alpha}, \Psi_{\gamma} \in C(J, \mathbb{R})$ be increasing functions, and there exist $\Omega_{\Psi_{\alpha}}, \Omega_{\Psi_{\gamma}}>0$ such that, for each $\sigma \in J$, the inequalities

$$
{ }_{H} \mathfrak{J}^{\alpha} \Psi_{\alpha}(\sigma) \leq \Omega_{\Psi_{\alpha}} \Psi_{\alpha}(\sigma){ }_{H} \mathfrak{J}^{\gamma} \Psi_{\gamma}(\sigma) \leq \Omega_{\Psi_{\gamma}} \Psi_{\gamma}(\sigma)
$$

hold. 


\section{Preliminaries}

In this section, we present some useful definitions, lemmas, and theorems, which will be used throughout the manuscript lenleadertwodots.

Definition 1 (see [31]). The Hadamard fractional integral of order, of function $\mathrm{u}(\sigma)$, is defined by

$$
{ }_{H} \mathfrak{\Im}^{\alpha} \mathrm{u}(\sigma)=\frac{1}{\Gamma(\alpha)} \int_{1}^{\sigma}\left(\ln \frac{\sigma}{\eta}\right)^{\alpha-1} \mathrm{u}(\eta) \frac{\mathrm{d} \eta}{\eta}, \quad 1 \leq \sigma \leq 2,
$$

where $\Gamma(\cdot)$ is the gamma function.

Definition 2 (see [31]). The Hadamard fractional derivative of order $\alpha \in[n-1, n), n \in \mathbb{Z}^{+}$of function $\mathrm{u}(\sigma)$ is defined by

$$
{ }_{H} \mathfrak{D}^{\alpha} \mathrm{u}(\sigma)=\frac{1}{\Gamma(n-\alpha)}\left(\sigma \frac{\mathrm{d}}{\mathrm{d} \sigma}\right)^{n} \int_{n}^{\sigma}\left(\ln \frac{\sigma}{\eta}\right)^{n-\alpha+1} \mathrm{u}(\eta) \frac{d \eta}{\eta},
$$$$
1 \leq \sigma \leq 2
$$

where $\Gamma(\cdot)$ is the gamma function.

Lemma 1 (see [31]). Let $\alpha>0$ and $\mathrm{u}$ be any function; then, the homogeneous differential equation along with Hadamard fractional order ${ }_{H} \mathfrak{D}^{\alpha} \mathrm{u}(\sigma)=0$ has a solution

$$
\mathrm{u}(\sigma)=\sum_{i=1}^{n} c_{i}(\ln \sigma)^{\alpha-i}
$$

and the following formula holds:

$$
{ }_{H} \mathfrak{J}_{H}^{\alpha} \mathfrak{D}^{\alpha} \mathrm{u}(\sigma)=\mathrm{u}(\sigma)+\sum_{i=1}^{n} c_{i}(\ln \sigma)^{\alpha-i},
$$

where $c_{i} \in \mathbb{R}, i=1,2, \ldots, n$, and $n-1<\alpha<n$.

Theorem 1 (see [32]) (Arzela-Ascoli's Theorem). Let $B \subset C(J, \mathbb{R})$ be relatively compact, and

(A) $B$ is a uniformly bounded set such that there exists $>0$ with

$$
\|\phi\|=\sup _{y \in J}|\phi(y)|<\Phi, \quad \text { for every } \phi \in B .
$$

(B) $B$ is an equicontinuous set, i.e., for every $\epsilon>0$, there exists $\delta>0$ such that, for any

$$
\begin{aligned}
& z, \bar{z} \in J, \\
& |z-\bar{z}| \leq \delta \Rightarrow|\phi(z)-\phi(\bar{z})| \leq \varepsilon,
\end{aligned}
$$

Theorem 2 (see [33]) (Banach Fixed-Point Theorem). Let $\mathscr{B}$ be a nonempty closed subset of a Banach space $\mathbb{X}$. Then, any contraction mapping $\mathfrak{T}$ from $\mathbb{X}$ into itself has a unique fixed point.

Theorem 3 (see [33]) (Schaefer's Fixed-Point Theorem). Let $\mathbb{X}$ be a Banach space. Suppose the operator $\mathfrak{T}: \mathbb{X} \longrightarrow \mathbb{X}$ is a continuous compact mapping (or completely continuous). Moreover, suppose

$$
\mathscr{B}=\{\mathrm{u} \in \mathbb{X} \mid \mathrm{u}=\delta \mathfrak{T} \mathrm{u}, \quad 0<\delta<1\},
$$

is a bounded set. Then, $\mathscr{B}$ has at least one fixed point in $\mathbb{X}$.

Lemma 2. Let $\mathbb{X}=\{\mathrm{u}(\sigma): \mathrm{u} \in C(J, \mathbb{R})\}$ be a Banach space endowed with the norm $\|\mathrm{u}\|_{\mathbb{X}}=\sup \{|\mathrm{u}(\sigma)|: \sigma \in J\}$. Similarly, the norm defined on the product space $\mathbb{X} \times \mathbb{X}$ is $\|(\mathrm{u}, \mathrm{v})\|_{\mathbb{X} \times \mathbb{X}}=\|\mathrm{u}\|_{\mathbb{X}}+\|\mathrm{v}\|_{\mathbb{X}}$. Obviously, $\left(\mathbb{X} \times \mathbb{X},\|(\mathrm{u}, \mathrm{v})\|_{\mathbb{X} \times \mathbb{X}}\right)$ is a Banach space. Also, the cone $\mathscr{C} \subset \mathbb{X} \times \mathbb{X}$ is defined as

$$
\mathscr{C}=\{(\mathrm{u}, \mathrm{v}) \in \mathbb{X} \times \mathbb{X}: \quad \mathrm{u}(\sigma) \geq 0, \mathrm{v}(\sigma) \geq 0\} .
$$

Theorem 4 (see [34]). Let $\mathbb{X}$ be a Banach space containing a cone $\mathscr{C} . \mathscr{B} \subset \mathscr{C}$ with $0 \in \mathscr{B}$ is a relatively open set, and the operator $\mathfrak{T}: \mathscr{B} \longrightarrow \mathscr{B}$ is completely continuous. Then, one of the following conditions holds:

(1) There exist $(\mathrm{u}, \mathrm{v}) \in \partial \mathscr{B}$ and $\delta \in(0,1)$ such that $(\mathrm{u}, \mathrm{v})=\delta \mathfrak{T}(\mathrm{u}, \mathrm{v})$

(2) $\mathfrak{I}$ has a fixed point in $\mathscr{B}$

\section{Existence and Uniqueness}

In this section, we give the existence and uniqueness of solutions of (5) and its coupled system (7).

3.1. Existence and Uniqueness Solution for System (5). Our first result is stated as follows.

Lemma 3. Let us assume that $\mathbf{f} \in C(J, \mathbb{R})$ and $\Delta>0$; then, the fractional differential equation

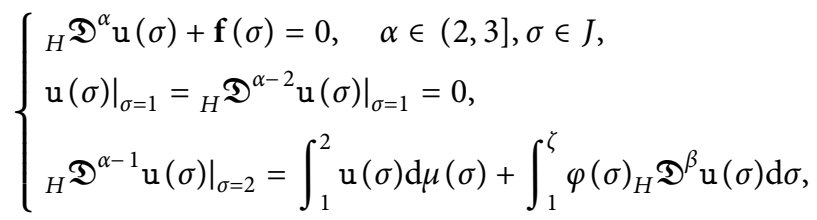

has a solution

$$
\mathrm{u}(\sigma)=\int_{1}^{2} \mathbb{G}(\sigma, \eta) f(\eta) \frac{\mathrm{d} \eta}{\eta}, \quad \sigma \in J
$$

where 


$$
\begin{aligned}
& \mathbb{G}(\sigma, \eta)=\mathbb{G}_{1}(\sigma, \eta)+\mathbb{G}_{2}(\sigma, \eta)+\mathbb{G}_{3}(\sigma, \eta), \\
& \mathbb{G}_{1}(\sigma, \eta)= \begin{cases}\frac{(\ln \sigma)^{\alpha-1}-(\ln \sigma / \eta)^{\alpha-1}}{\Gamma(\alpha)}, & 1 \leq \eta \leq \sigma \leq 2, \\
\frac{(\ln \sigma)^{\alpha-1}}{\Gamma(\alpha)}, & 1 \leq \sigma \leq \eta \leq 2,\end{cases} \\
& \mathbb{G}_{2}(\sigma, \eta)=\frac{(\ln \sigma)^{\alpha-1}}{\Delta} \int_{1}^{\zeta} \varphi(\sigma) \mathbb{Q}_{1}(\sigma, \eta) \mathrm{d} \sigma, \\
& \mathbb{G}_{3}(\sigma, \eta)=\frac{\Gamma(\alpha-\beta)(\ln \sigma)^{\alpha-1}}{\Delta \Gamma(\alpha)} \int_{1}^{2} \mathbb{G}_{1}(\sigma, \eta) \mathrm{d} \mu(\sigma), \\
& \frac{(\ln \sigma)^{\alpha-1-\beta}-(\ln (\sigma / \eta))^{\alpha-1-\beta}}{\Gamma(\alpha)}, 1 \leq \eta \leq \sigma \leq 2, \\
& \mathbb{Q}_{1}(\sigma, \eta)= \begin{cases}\frac{(\ln \sigma)^{\alpha-1-\beta}}{\Gamma(\alpha)}, & 1 \leq \sigma \leq \eta \leq 2 .\end{cases}
\end{aligned}
$$

Proof. Consider

$$
\begin{aligned}
& { }_{H} \mathfrak{D}^{\alpha} \mathrm{u}(\sigma)+\mathbf{f}(\sigma)=0, \\
& \alpha \in(2,3], \quad \sigma \in J .
\end{aligned}
$$

For $\sigma \in J$, Lemma 1 gives

$$
\begin{aligned}
\mathrm{u}(\sigma)= & c_{1}(\ln \sigma)^{\alpha-1}+c_{2}(\ln \sigma)^{\alpha-2}+c_{3}(\ln \sigma)^{\alpha-3} \\
& -\frac{1}{\Gamma(\alpha)} \int_{1}^{\sigma}\left(\ln \frac{\sigma}{\eta}\right)^{\alpha-1} \mathbf{f}(\eta) \frac{\mathrm{d} \eta}{\eta},
\end{aligned}
$$

for some $c_{i} \in \mathbb{R}, i=1,2,3$. By the condition $\left.\mathrm{u}(\sigma)\right|_{\sigma=1}=0$, we have $c_{3}=0$. Hence,

$$
\begin{aligned}
\mathrm{u}(\sigma)= & c_{1}(\ln \sigma)^{\alpha-1}+c_{2}(\ln \sigma)^{\alpha-2} \\
& -\frac{1}{\Gamma(\alpha)} \int_{1}^{\sigma}\left(\ln \frac{\sigma}{\eta}\right)^{\alpha-1} \mathbf{f}(\eta) \frac{\mathrm{d} \eta}{\eta} .
\end{aligned}
$$
get

Now, considering the condition $\left.{ }_{H} \mathfrak{D}^{\alpha-2} \mathrm{u}(\sigma)\right|_{\sigma=1}=0$, we

$$
\begin{aligned}
{ }_{H} \mathfrak{D}^{\alpha-2} u(\sigma)= & c_{1 H} \mathfrak{D}^{\alpha-2}(\ln \sigma)^{\alpha-1}+c_{2 H} \mathfrak{D}^{\alpha-2}(\ln \sigma)^{\alpha-2} \\
& -{ }_{H} \mathfrak{D}^{\alpha-2}{ }_{H} \mathfrak{J}^{\alpha} \mathbf{f}(\sigma), \\
= & c_{1} \frac{\Gamma(\alpha)}{\Gamma(2)}(\ln \sigma)+c_{2} \frac{\Gamma(\alpha-1)}{\Gamma(1)}-{ }_{H} \mathfrak{J}^{2} \mathbf{f}(\sigma),
\end{aligned}
$$

in view of ${ }_{H} \mathfrak{J}^{2} \mathbf{f}(\sigma) \longrightarrow 0$ as $\sigma \longrightarrow 0$, so we must set $c_{2}=0$. Then, we have

$$
\mathrm{u}(\sigma)=c_{1}(\ln \sigma)^{\alpha-1}-\frac{1}{\Gamma(\alpha)} \int_{1}^{\sigma}\left(\ln \frac{\sigma}{\eta}\right)^{\alpha-1} \mathbf{f}(\eta) \frac{\mathrm{d} \eta}{\eta} .
$$

Furthermore, we obtain

$$
\begin{aligned}
{ }_{H} \mathfrak{D}^{\alpha-1} \mathrm{u}(\sigma)= & c_{1 H} \mathfrak{D}^{\alpha-1}(\ln \sigma)^{\alpha-1}-{ }_{H} \mathfrak{D}^{\alpha-1}{ }_{H} \mathfrak{J}^{\alpha} f(\sigma), \\
= & c_{1} \Gamma(\alpha)-\int_{1}^{\sigma} \mathbf{f}(\eta) \frac{\mathrm{d} \eta}{\eta}, \\
{ }_{H} \mathfrak{D}^{\beta} \mathrm{u}(\sigma)= & c_{1 H} \mathfrak{D}^{\beta}(\ln \sigma)^{\alpha-1}-{ }_{H} \mathfrak{D}^{\beta}{ }_{H} \mathfrak{J}^{\alpha} \mathbf{f}(\sigma), \\
= & c_{1} \frac{\Gamma(\alpha)}{\Gamma(\alpha-\beta)}(\ln \sigma)^{\alpha-1-\beta}-\frac{1}{\Gamma(\alpha-\beta)} \\
& \cdot \int_{1}^{\sigma}\left(\ln \frac{\sigma}{\eta}\right)^{\alpha-1-\beta} \mathbf{f}(\eta) \frac{\mathrm{d} \eta}{\eta} .
\end{aligned}
$$

Then, we obtain

$$
\begin{aligned}
& \int_{1}^{2} \mathrm{u}(\sigma) \mathrm{d} \mu(\sigma)+\int_{1}^{\zeta} \varphi(\sigma)_{H} \mathfrak{D}^{\beta} \mathrm{u}(\sigma) \mathrm{d} \sigma, \\
& =c_{1} \int_{1}^{2}(\ln \sigma)^{\alpha-1} \mathrm{~d} \mu(\sigma)+c_{1} \frac{\Gamma(\alpha)}{\Gamma(\alpha-\beta)} \int_{1}^{\zeta} \varphi(\sigma)(\ln \sigma)^{\alpha-1-\beta} \mathrm{d} \sigma \\
& \quad-\frac{1}{\Gamma(\alpha)} \int_{1}^{2} \int_{1}^{\sigma}\left(\ln \frac{\sigma}{\eta}\right)^{\alpha-1} \mathbf{f}(\eta) \frac{\mathrm{d} \eta}{\eta} \mathrm{d} \mu(\sigma)-\frac{1}{\Gamma(\alpha-\beta)} \int_{1}^{\zeta} \varphi(\sigma) \int_{1}^{\sigma}\left(\ln \frac{\sigma}{\eta}\right)^{\alpha-1-\beta} \mathbf{f}(\eta) \frac{\mathrm{d} \eta}{\eta} \mathrm{d} \sigma .
\end{aligned}
$$


Applying the 2nd condition, we get

$$
\begin{aligned}
c_{1}= & \frac{\int_{1}^{2} \mathbf{f}(\eta)(\mathrm{d} \eta / \eta)}{\Gamma(\alpha)-(\Gamma(\alpha) / \Gamma(\alpha-\beta)) \int_{1}^{\zeta} \varphi(\sigma)(\ln \sigma)^{\alpha-1-\beta} \mathrm{d} \sigma-\int_{1}^{2}(\ln \sigma)^{\alpha-1} \mathrm{~d} \mu(\sigma)} \\
& -\frac{\int_{1}^{\zeta} \varphi(\sigma) \int_{1}^{\sigma}(\ln (\sigma / \eta))^{\alpha-1-\beta} \mathbf{f}(\eta)(\mathrm{d} \eta / \eta) \mathrm{d} \sigma}{\Gamma(\alpha) \Gamma(\alpha-\beta)-\Gamma(\alpha) \int_{1}^{\zeta} \varphi(\sigma)(\ln \sigma)^{\alpha-1-\beta} \mathrm{d} \sigma-\Gamma(\alpha-\beta) \int_{1}^{2}(\ln \sigma)^{\alpha-1} \mathrm{~d} \mu(\sigma)} \\
& -\frac{\int_{1}^{2} \int_{1}^{\sigma}(\ln (\sigma / \eta))^{\alpha-1} \mathbf{f}(\eta)(\mathrm{d} \eta / \eta) \mathrm{d} \mu(\sigma)}{\Gamma^{2}(\alpha)-\left(\Gamma^{2}(\alpha) / \Gamma(\alpha-\beta)\right) \int_{1}^{\zeta} \varphi(\sigma)(\ln \sigma)^{\alpha-1-\beta} \mathrm{d} \sigma-\Gamma(\alpha) \int_{1}^{2}(\ln \sigma)^{\alpha-1} \mathrm{~d} \mu(\sigma)} .
\end{aligned}
$$

Substituting (37) into (34), we get

$$
\begin{aligned}
& \mathrm{u}(\sigma)=\frac{(\ln \sigma)^{\alpha-1} \int_{1}^{2} \mathbf{f}(\eta)(\mathrm{d} \eta / \eta)}{\Gamma(\alpha) \Gamma(\alpha-\beta)-\Gamma(\alpha) \int_{1}^{\zeta} \varphi(\sigma)(\ln \sigma)^{\alpha-1-\beta} \mathrm{d} \sigma-\Gamma(\alpha-\beta) \int_{1}^{2}(\ln \sigma)^{\alpha-1} \mathrm{~d} \mu(\sigma)} \\
& \times\left(\Gamma(\alpha-\beta)-\int_{1}^{\zeta} \varphi(\sigma)(\ln \sigma)^{\alpha-1-\beta} \mathrm{d} \sigma-\frac{\Gamma(\alpha-\beta)}{\Gamma(\alpha)} \int_{1}^{2}(\ln \sigma)^{\alpha-1} \mathrm{~d} \mu(\sigma)\right. \\
& \left.+\int_{1}^{\zeta} \varphi(\sigma)(\ln \sigma)^{\alpha-1-\beta} \mathrm{d} \sigma+\frac{\Gamma(\alpha-\beta)}{\Gamma(\alpha)} \int_{1}^{2}(\ln \sigma)^{\alpha-1} \mathrm{~d} \mu(\sigma)\right)-\frac{1}{\Gamma(\alpha)} \int_{1}^{\sigma}\left(\ln \frac{\sigma}{\eta}\right)^{\alpha-1} \mathbf{f}(\eta) \frac{\mathrm{d} \eta}{\eta} \\
& -\frac{(\ln \sigma)^{\alpha-1} \int_{1}^{\zeta} \varphi(\sigma) \int_{1}^{\sigma}(\ln (\sigma / \eta))^{\alpha-1-\beta} \mathbf{f}(\eta)(\mathrm{d} \eta / \eta) \mathrm{d} \sigma}{\Gamma(\alpha) \Gamma(\alpha-\beta)-\Gamma(\alpha) \int_{1}^{\zeta} \varphi(\sigma)(\ln \sigma)^{\alpha-1-\beta} \mathrm{d} \sigma-\Gamma(\alpha-\beta) \int_{1}^{2}(\ln \sigma)^{\alpha-1} \mathrm{~d} \mu(\sigma)} \\
& -\frac{(\ln \sigma)^{\alpha-1} \int_{1}^{2} \int_{1}^{\sigma}(\ln (\sigma / \eta))^{\alpha-1} \mathbf{f}(\eta)(\mathrm{d} \eta / \eta) \mathrm{d} \mu(\sigma)}{\Gamma^{2}(\alpha)-\left(\Gamma^{2}(\alpha) / \Gamma(\alpha-\beta)\right) \int_{1}^{\zeta} \varphi(\sigma)(\ln \sigma)^{\alpha-1-\beta} \mathrm{d} \sigma-\Gamma(\alpha) \int_{1}^{2}(\ln \sigma)^{\alpha-1} \mathrm{~d} \mu(\sigma)}, \\
& =\frac{(\ln \sigma)^{\alpha-1}}{\Gamma(\alpha-\beta)-\int_{1}^{\zeta} \varphi(\sigma)(\ln \sigma)^{\alpha-1-\beta} \mathrm{d} \sigma-(\Gamma(\alpha-\beta) / \Gamma(\alpha)) \int_{1}^{2}(\ln \sigma)^{\alpha-1} \mathrm{~d} \mu(\sigma)} \\
& \times \frac{1}{\Gamma(\alpha)}\left(\int_{1}^{\zeta} \int_{1}^{2} \varphi(\sigma)(\ln \sigma)^{\alpha-1-\beta} \mathbf{f}(\eta) \frac{\mathrm{d} \eta}{\eta} \mathrm{d} \sigma-\int_{1}^{\zeta} \int_{1}^{\sigma} \varphi(\sigma)\left(\ln \frac{\sigma}{\eta}\right)^{\alpha-1-\beta} \mathbf{f}(\eta) \frac{\mathrm{d} \eta}{\eta} \mathrm{d} \sigma\right) \\
& +\frac{\Gamma(\alpha-\beta)(\ln \sigma)^{\alpha-1}}{\Gamma(\alpha) \Gamma(\alpha-\beta)-\Gamma(\alpha) \int_{1}^{\zeta} \varphi(\sigma)(\ln \sigma)^{\alpha-1-\beta} \mathrm{d} \sigma-\Gamma(\alpha-\beta) \int_{1}^{2}(\ln \sigma)^{\alpha-1} \mathrm{~d} \mu(\sigma)} \\
& \times \frac{1}{\Gamma(\alpha)} \int_{1}^{2}(\ln \sigma)^{\alpha-1} \int_{1}^{2} \mathbf{f}(\eta) \frac{\mathrm{d} \eta}{\eta} \mathrm{d} \mu(\sigma) \\
& -\frac{(\ln \sigma)^{\alpha-1}}{\Gamma^{2}(\alpha) \Gamma(\alpha-\beta)-\Gamma^{2}(\alpha) \int_{1}^{\zeta} \varphi(\sigma)(\ln \sigma)^{\alpha-1-\beta} \mathrm{d} \sigma-\Gamma(\alpha) \Gamma(\alpha-\beta) \int_{1}^{2}(\ln \sigma)^{\alpha-1} \mathrm{~d} \mu(\sigma)} \\
& \times \int_{1}^{2} \int_{1}^{\sigma}\left(\ln \frac{\sigma}{\eta}\right)^{\alpha-1} \mathbf{f}(\eta) \frac{\mathrm{d} \eta}{\eta} \mathrm{d} \mu(\sigma)+\int_{1}^{2} \mathbb{G}_{1}(\sigma, \eta) f(\eta) \frac{\mathrm{d} \eta}{\eta},
\end{aligned}
$$




$$
\begin{aligned}
= & \frac{(\ln \sigma)^{\alpha-1}}{\Gamma(\alpha-\beta)-\int_{1}^{\zeta} \varphi(\sigma)(\ln \sigma)^{\alpha-1-\beta} \mathrm{d} \sigma-(\Gamma(\alpha-\beta) / \Gamma(\alpha)) \int_{1}^{2}(\ln \sigma)^{\alpha-1} \mathrm{~d} \mu(\sigma)} \\
& \times \frac{1}{\Gamma(\alpha)}\left[\int_{1}^{\zeta} \int_{1}^{\sigma} \varphi(\sigma)\left((\ln \sigma)^{\alpha-1-\beta}-\left(\ln \frac{\sigma}{\eta}\right)^{\alpha-1-\beta}\right) \mathbf{f}(\eta) \frac{\mathrm{d} \eta}{\eta} \mathrm{d} \sigma+\int_{1}^{\zeta} \int_{\sigma}^{2} \varphi(\sigma)(\ln \sigma)^{\alpha-1-\beta} \mathbf{f}(\eta) \frac{\mathrm{d} \eta}{\eta} \mathrm{d} \sigma\right] \\
& +\frac{\Gamma(\alpha-\beta)(\ln \sigma)^{\alpha-1}}{\Gamma(\alpha) \Gamma(\alpha-\beta)-\Gamma(\alpha) \int_{1}^{\zeta} \varphi(\sigma)(\ln \sigma)^{\alpha-1-\beta} \mathrm{d} \sigma-\Gamma(\alpha-\beta) \int_{1}^{2}(\ln \sigma)^{\alpha-1} \mathrm{~d} \mu(\sigma)} \\
& \times \frac{1}{\Gamma(\alpha)}\left[\int_{1}^{2} \int_{1}^{\sigma}\left((\ln \sigma)^{\alpha-1}-\left(\ln \frac{\sigma}{\eta}\right)^{\alpha-1}\right) \mathbf{f}(\eta) \frac{\mathrm{d} \eta}{\eta} \mathrm{d} \mu(\sigma)+\int_{1}^{2} \int_{\sigma}^{2}(\ln \sigma)^{\alpha-1} \mathbf{f}(\eta) \frac{\mathrm{d} \eta}{\eta} \mathrm{d} \mu(\sigma)\right] \\
& +\int_{1}^{2} \mathbb{G}_{1}(\sigma, \eta) \mathbf{f}(\eta) \frac{\mathrm{d} \eta}{\eta}=\frac{(\ln \sigma)^{\alpha-1}}{\Delta} \int_{1}^{\zeta} \varphi(\sigma) \int_{1}^{2} \mathbb{Q}_{1}(\sigma, \eta) \mathbf{f}(\eta) \frac{\mathrm{d} \eta}{\eta} \mathrm{d} \sigma \\
& +\int_{1}^{2} \mathbb{G}_{1}(\sigma, \eta) \mathbf{f}(\eta) \frac{\mathrm{d} \eta}{\eta}+\frac{\Gamma(\alpha-\beta)(\ln \sigma)^{\alpha-1}}{\Delta \Gamma(\alpha)} \int_{1}^{2} \int_{1}^{2} \mathbb{G}_{1}(\sigma, \eta) \mathbf{f}(\eta) \frac{\mathrm{d} \eta}{\eta} \mathrm{d} \mu(\sigma), \\
= & \int_{1}^{2}\left(\mathbb{G}_{1}(\sigma, \eta)+\mathbb{G}_{2}(\sigma, \eta)+\mathbb{G}_{3}(\sigma, \eta)\right) \mathbf{f}(\eta) \frac{\mathrm{d} \eta}{\eta}=\int_{1}^{2} \mathbb{G}(\sigma, \eta) \mathbf{f}(\eta) \frac{\mathrm{d} \eta}{\eta} .
\end{aligned}
$$

By replacing $\mathbf{f}(\sigma)=\phi\left(\sigma, \mathrm{u}(\sigma){ }_{H} \mathfrak{D}^{\alpha} \mathrm{u}(\sigma)\right)$ in Lemma 3, we get the integral equation of problem (5) as

$$
\mathrm{u}(\sigma)=\int_{1}^{2} \mathbb{G}(\sigma, \eta) \phi\left(\eta, u(\eta), \mathfrak{D}^{\alpha} u(\eta)\right) \frac{\mathrm{d} \eta}{\eta} .
$$

(1) $\mathbb{G}(\sigma, \eta) \geqslant 0$ for all $(\sigma, \eta) \in J$

(2) $\mathbb{G}(\sigma, \eta)$ is continuous over $J \times J$

(3) $\max _{\sigma \in J} \int_{1}^{2}|\mathbb{G}(\sigma, \eta)| d \eta / \eta \leqslant \mathscr{Y}$,

where

Lemma 4. Green's function $\mathbb{G}(\sigma, \eta)$, which is obtained in (39), has the following properties:

$$
\begin{aligned}
\mathscr{Y}= & \frac{(\ln 2)^{\alpha-1}}{\nabla \Gamma(\alpha+1)}\left(\alpha \mathfrak{E} \zeta(\ln 2) \sum_{k=0}^{\alpha-1-\beta}(-1)^{\alpha-1-\beta-k} \frac{(\alpha-1-\beta) !}{k !}(\ln \zeta)^{k}\right. \\
& +\frac{\alpha \mathfrak{E} \zeta}{\alpha-\beta} \sum_{k=0}^{\alpha-\beta}(-1)^{\alpha-\beta-k} \frac{(\alpha-\beta) !}{k !}(\ln \zeta)^{k} \\
& \left.+\mathfrak{P}(\ln \rho)^{\alpha-1} \Gamma(\alpha-\beta)(\alpha(\ln 2)+(\ln \rho))+\nabla(\alpha(\ln 2)+(\ln 2))\right), \\
\nabla= & \Gamma(\alpha-\beta)+\mathfrak{E} \zeta \sum_{k=0}^{\alpha-1-\beta}(-1)^{\alpha-1-\beta-k} \frac{(\alpha-1-\beta) !}{k !}(\ln \zeta)^{k}+\frac{(\ln \rho)^{\alpha-1} \mathfrak{P} \Gamma(\alpha-\beta)}{\Gamma(\alpha)}
\end{aligned}
$$


(3) Since we know that Green's function of the considered problem is in the form

$$
\begin{aligned}
\int_{1}^{2}|\mathbb{G}(\sigma, \eta)| \frac{\mathrm{d} \eta}{\eta}= & \mid \frac{(\ln \sigma)^{\alpha-1}}{\Delta \Gamma(\alpha)}\left[\int_{1}^{\zeta} \int_{1}^{2} \varphi(\sigma)(\ln \sigma)^{\alpha-1-\beta} \frac{\mathrm{d} \eta}{\eta} \mathrm{d} \sigma-\int_{1}^{\zeta} \int_{1}^{\sigma} \varphi(\sigma)\left(\ln \frac{\sigma}{\eta}\right)^{\alpha-1-\beta} \frac{\mathrm{d} \eta}{\eta} \mathrm{d} \sigma\right] \\
& +\frac{(\ln \sigma)^{\alpha-1} \Gamma(\alpha-\beta)}{\Delta \Gamma^{2}(\alpha)}\left[\int_{1}^{2} \int_{1}^{2}(\ln \sigma)^{\alpha-1} \frac{\mathrm{d} \eta}{\eta} \mathrm{d} \mu(\sigma)-\int_{1}^{2} \int_{1}^{\sigma}\left(\ln \frac{\sigma}{\eta}\right)^{\alpha-1} \frac{\mathrm{d} \eta}{\eta} \mathrm{d} \mu(\sigma)\right] \\
& +\frac{(\ln \sigma)^{\alpha-1}}{\Gamma(\alpha)} \int_{1}^{2} \frac{\mathrm{d} \eta}{\eta}-\frac{1}{\Gamma(\alpha)} \int_{1}^{\sigma}\left(\ln \frac{\sigma}{\eta}\right)^{\alpha-1} \frac{\mathrm{d} \eta}{\eta} \mid \\
\leq & \frac{(\ln \sigma)^{\alpha-1}}{|\Delta| \Gamma(\alpha)}\left[\int_{1}^{\zeta} \int_{1}^{2}|\varphi(\sigma)|(\ln \sigma)^{\alpha-1-\beta} \frac{\mathrm{d} \eta}{\eta} \mathrm{d} \sigma+\int_{1}^{\zeta} \int_{1}^{\sigma}|\varphi(\sigma)|\left(\ln \frac{\sigma}{\eta}\right)^{\alpha-1-\beta} \mid \frac{\mathrm{d} \eta}{\eta} \mathrm{d} \sigma\right] \\
& +\frac{(\ln \sigma)^{\alpha-1} \Gamma(\alpha-\beta)}{|\Delta| \Gamma^{2}(\alpha)}\left[\int_{1}^{2} \int_{1}^{2}(\ln \sigma)^{\alpha-1} \frac{\mathrm{d} \eta}{\eta} \mathrm{d} \mu(\sigma)+\int_{1}^{2} \int_{1}^{\sigma}\left|\left(\ln \frac{\sigma}{\eta}\right)^{\alpha-1}\right| \frac{\mathrm{d} \eta}{\eta} \mathrm{d} \mu(\sigma)\right] \\
& +\frac{(\ln \sigma)^{\alpha-1}}{\Gamma(\alpha)} \int_{1}^{2} \frac{\mathrm{d} \eta}{\eta}+\frac{1}{\Gamma(\alpha)} \int_{1}^{\sigma}\left|\left(\ln \frac{\sigma}{\eta}\right)^{\alpha-1}\right| \frac{\mathrm{d} \eta}{\eta},
\end{aligned}
$$

using $\left(\mathbf{M}_{5}\right)$ and mean-value theorem [35] with $\rho \in[1,2]$ and $\mu(2)=\mathfrak{P}>0$, we get

$$
\begin{aligned}
\max _{\sigma \in J} \int_{1}^{2}|\mathbb{G}(\sigma, \eta)| \frac{\mathrm{d} \eta}{\eta} \leq & \frac{(\ln 2)^{\alpha-1}}{\nabla \Gamma(\alpha+1)}\left(\alpha \mathfrak{E} \zeta(\ln 2) \sum_{k=0}^{\alpha-1-\beta}(-1)^{\alpha-1-\beta-k} \frac{(\alpha-1-\beta) !}{k !}(\ln \zeta)^{k}\right. \\
& +\frac{\alpha \mathfrak{E} \zeta}{\alpha-\beta} \sum_{k=0}^{\alpha-\beta}(-1)^{\alpha-\beta-k} \frac{(\alpha-\beta) !}{k !}(\ln \zeta)^{k} \\
& \left.+\mathfrak{P}(\ln \rho)^{\alpha-1} \Gamma(\alpha-\beta)(\alpha(\ln 2)+(\ln \rho))+\nabla(\alpha(\ln 2)+(\ln 2))\right)=\mathscr{Y} .
\end{aligned}
$$

Hence, the proof of (3) is complete.

If $u$ is the solution of the given system (5) and $\sigma \in J$, then

$$
\begin{aligned}
\mathrm{u}(\sigma)= & \frac{(\ln \sigma)^{\alpha-1}}{\Delta \Gamma(\alpha)} \int_{1}^{\zeta} \int_{1}^{2} \varphi(\sigma)(\ln \sigma)^{\alpha-1-\beta} \phi(\eta, \mathrm{u}(\eta), \mathrm{z}(\eta)) \frac{\mathrm{d} \eta}{\eta} \mathrm{d} \sigma \\
& -\frac{(\ln \sigma)^{\alpha-1}}{\Delta \Gamma(\alpha)} \int_{1}^{\zeta} \int_{1}^{\sigma} \varphi(\sigma)\left(\ln \frac{\sigma}{\eta}\right)^{\alpha-1-\beta} \phi(\eta, \mathrm{u}(\eta), \mathrm{z}(\eta)) \frac{\mathrm{d} \eta}{\eta} \mathrm{d} \sigma \\
& +\frac{(\ln \sigma)^{\alpha-1} \Gamma(\alpha-\beta)}{\Delta \Gamma^{2}(\alpha)} \int_{1}^{2} \int_{1}^{2}(\ln \sigma)^{\alpha-1} \phi(\eta, \mathrm{u}(\eta), \mathrm{z}(\eta)) \frac{\mathrm{d} \eta}{\eta} \mathrm{d} \mu(\sigma) \\
& -\frac{(\ln \sigma)^{\alpha-1} \Gamma(\alpha-\beta)}{\Delta \Gamma^{2}(\alpha)} \int_{1}^{2} \int_{1}^{\sigma}\left(\ln \frac{\sigma}{\eta}\right)^{\alpha-1} \phi(\eta, \mathrm{u}(\eta), \mathrm{z}(\eta)) \frac{\mathrm{d} \eta}{\eta} \mathrm{d} \mu(\sigma) \\
& +\frac{(\ln \sigma)^{\alpha-1}}{\Gamma(\alpha)} \int_{1}^{2} \phi(\eta, \mathrm{u}(\eta), \mathrm{z}(\eta)) \frac{\mathrm{d} \eta}{\eta}-\frac{1}{\Gamma(\alpha)} \int_{1}^{\sigma}\left(\ln \frac{\sigma}{\eta}\right)^{\alpha-1} \phi(\eta, \mathrm{u}(\eta), \mathrm{z}(\eta)) \frac{\mathrm{d} \eta}{\eta},
\end{aligned}
$$


where

We define an operator $\mathfrak{T}: \mathbb{X} \longrightarrow \mathbb{X}$ as

$$
z(\sigma)={ }_{H} \mathfrak{D}^{\alpha} \mathrm{u}(\sigma) .
$$

$$
\begin{aligned}
\mathfrak{T} \mathrm{u}(\sigma)= & -\frac{(\ln \sigma)^{\alpha-1}}{\Delta \Gamma(\alpha)}\left[\int_{1}^{\zeta} \int_{1}^{2} \varphi(\sigma)(\ln \sigma)^{\alpha-1-\beta} \mathrm{z}(\eta) \frac{\mathrm{d} \eta}{\eta} \mathrm{d} \sigma-\int_{1}^{\zeta} \int_{1}^{\sigma} \varphi(\sigma)\left(\ln \frac{\sigma}{\eta}\right)^{\alpha-1-\beta} \mathrm{z}(\eta) \frac{\mathrm{d} \eta}{\eta} \mathrm{d} \sigma\right] \\
& -\frac{(\ln \sigma)^{\alpha-1} \Gamma(\alpha-\beta)}{\Delta \Gamma^{2}(\alpha)}\left[\int_{1}^{2} \int_{1}^{2}(\ln \sigma)^{\alpha-1} \mathrm{z}(\eta) \frac{\mathrm{d} \eta}{\eta} \mathrm{d} \mu(\sigma)-\int_{1}^{2} \int_{1}^{\sigma}\left(\ln \frac{\sigma}{\eta}\right)^{\alpha-1} \mathrm{z}(\eta) \frac{\mathrm{d} \eta}{\eta} \mathrm{d} \mu(\sigma)\right] \\
& -\frac{(\ln \sigma)^{\alpha-1}}{\Gamma(\alpha)} \int_{1}^{2} \mathrm{z}(\eta) \frac{\mathrm{d} \eta}{\eta}+\frac{1}{\Gamma(\alpha)} \int_{1}^{\sigma}\left(\ln \frac{\sigma}{\eta}\right)^{\alpha-1} \mathrm{z}(\eta) \frac{\mathrm{d} \eta}{\eta},
\end{aligned}
$$

where $\mathrm{z} \in C(J, \mathbb{R})$ such that

$$
\mathbf{z}(\sigma)=-\phi(\sigma, u(\sigma), \mathbf{z}(\sigma)) .
$$

Theorem 5. Let $\left(\mathbf{M}_{2}\right)-\left(\mathbf{M}_{5}\right)$ hold. Then, the operator $\mathfrak{I}$, defined in (45), is compact.
Proof. To show that the operator $\mathfrak{I}$ is compact, we follow several steps.

Step 1: we consider a sequence $\left\{\mathrm{u}_{n}\right\}$ such that $\mathrm{u}_{n} \longrightarrow \mathrm{u}$ in $\mathbb{X}$; then, for each $\sigma \in J$, we have

$$
\begin{aligned}
\left|\mathfrak{I}\left(\mathrm{u}_{n}\right)(\sigma)-\mathfrak{I}(\mathrm{u})(\sigma)\right|=\mid & -\frac{(\ln \sigma)^{\alpha-1}}{\Delta \Gamma(\alpha)} \int_{1}^{\zeta} \int_{1}^{2} \varphi(\sigma)(\ln \sigma)^{\alpha-1-\beta}\left(\mathbf{z}_{n}(\eta)-\mathrm{z}(\eta)\right) \frac{\mathrm{d} \eta}{\eta} \mathrm{d} \sigma \\
& +\frac{(\ln \sigma)^{\alpha-1}}{\Delta \Gamma(\alpha)} \int_{1}^{\zeta} \int_{1}^{\sigma} \varphi(\sigma)\left(\ln \frac{\sigma}{\eta}\right)^{\alpha-1-\beta}\left(\mathbf{z}_{n}(\eta)-\mathbf{z}(\eta)\right) \frac{\mathrm{d} \eta}{\eta} \mathrm{d} \sigma \\
& -\frac{(\ln \sigma)^{\alpha-1} \Gamma(\alpha-\beta)}{\Delta \Gamma^{2}(\alpha)} \int_{1}^{2} \int_{1}^{2}(\ln \sigma)^{\alpha-1}\left(\mathbf{z}_{n}(\eta)-\mathbf{z}(\eta)\right) \frac{\mathrm{d} \eta}{\eta} \mathrm{d} \mu(\sigma) \\
& +\frac{(\ln \sigma)^{\alpha-1} \Gamma(\alpha-\beta)}{\Delta \Gamma^{2}(\alpha)} \int_{1}^{2} \int_{1}^{\sigma}\left(\ln \frac{\sigma}{\eta}\right)^{\alpha-1}\left(\mathbf{z}_{n}(\eta)-\mathbf{z}(\eta)\right) \frac{\mathrm{d} \eta}{\eta} \mathrm{d} \mu(\sigma) \\
& -\frac{(\ln \sigma)^{\alpha-1}}{\Gamma(\alpha)} \int_{1}^{2}\left(\mathbf{z}_{n}(\eta)-\mathbf{z}(\eta)\right) \frac{\mathrm{d} \eta}{\eta}+\frac{1}{\Gamma(\alpha)} \int_{1}^{\sigma}\left(\ln \frac{\sigma}{\eta}\right)^{\alpha-1}\left(\mathbf{z}_{n}(\eta)-\mathbf{z}(\eta)\right) \frac{\mathrm{d} \eta}{\eta} \mid \\
\leq & \frac{(\ln \sigma)^{\alpha-1}}{|\Delta| \Gamma(\alpha)} \int_{1}^{\zeta} \int_{1}^{2}|\varphi(\sigma)|(\ln \sigma)^{\alpha-1-\beta}\left|\mathbf{z}_{n}(\eta)-\mathbf{z}(\eta)\right| \frac{\mathrm{d} \eta}{\eta} \mathrm{d} \sigma \\
& +\frac{(\ln \sigma)^{\alpha-1}}{|\Delta| \Gamma(\alpha)} \int_{1}^{\zeta} \int_{1}^{\sigma}|\varphi(\sigma)|\left(\ln \frac{\sigma}{\eta}\right)^{\alpha-1-\beta}|| \mathbf{z}_{n}(\eta)-\mathbf{z}(\eta) \mid \frac{\mathrm{d} \eta}{\eta} \mathrm{d} \sigma \\
& +\frac{(\ln \sigma)^{\alpha-1} \Gamma(\alpha-\beta)}{|\Delta| \Gamma^{2}(\alpha)} \int_{1}^{2} \int_{1}^{2}(\ln \sigma)^{\alpha-1}\left|\mathbf{z}_{n}(\eta)-\mathbf{z}(\eta)\right| \frac{\mathrm{d} \eta}{\eta} \mathrm{d} \mu(\sigma) \\
& +\frac{(\ln \sigma)^{\alpha-1} \Gamma(\alpha-\beta)}{|\Delta| \Gamma^{2}(\alpha)} \int_{1}^{2} \int_{1}^{\sigma}\left|\left(\ln \frac{\sigma}{\eta}\right)^{\alpha-1}\right|\left|\mathbf{z}_{n}(\eta)-\mathbf{z}(\eta)\right| \frac{\mathrm{d} \eta}{\eta} \mathrm{d} \mu(\sigma) \\
& \frac{(\ln \sigma)^{\alpha-1}}{\Gamma(\alpha)} \int_{1}^{2}\left|\mathbf{z}_{n}(\eta)-\mathbf{z}(\eta)\right| \frac{\mathrm{d} \eta}{\eta}+\frac{1}{\Gamma(\alpha)} \int_{1}^{\sigma}\left|\left(\ln \frac{\sigma}{\eta}\right)^{\alpha-1}\right|\left|\mathbf{z}_{n}(\eta)-\mathbf{z}(\eta)\right| \frac{\mathrm{d} \eta}{\eta}
\end{aligned}
$$


From (46), we can write

$$
\left|\mathfrak{I}\left(\mathrm{u}_{n}\right)(\sigma)-\mathfrak{I}(\mathrm{u})(\sigma)\right| \longrightarrow 0, \quad \text { as } n \longrightarrow \infty .
$$

$$
\begin{gathered}
\mathbf{z}_{n}(\sigma)=-\phi\left(\sigma, \mathrm{u}_{n}(\sigma), \mathbf{z}_{n}(\sigma)\right), \\
\mathbf{z}(\sigma)=-\phi(\sigma, \mathrm{u}(\sigma), \mathbf{z}(\sigma)) .
\end{gathered}
$$

Now, by $\left(\mathbf{M}_{4}\right)$, we have

$$
\begin{aligned}
\left|\mathbf{z}_{n}(\sigma)-\mathbf{z}(\sigma)\right| & =\left|-\left(\phi\left(\sigma, \mathrm{u}_{n}(\sigma), \mathbf{z}_{n}(\sigma)\right)-\phi(\sigma, \mathrm{u}(\sigma), \mathbf{z}(\sigma))\right)\right| \\
& \leq \mathbf{Q}_{\phi}\left|\mathrm{u}_{n}(\sigma)-\mathrm{u}(\sigma)\right|+\mathbf{Q}_{\phi}^{*}\left|\mathbf{z}_{n}(\sigma)-\mathbf{z}(\sigma)\right|,
\end{aligned}
$$

This implies

$$
\left\|\mathfrak{T}\left(\mathrm{u}_{n}\right)-\mathfrak{T}(\mathrm{u})\right\|_{\mathbb{X}} \longrightarrow 0, \quad \text { as } n \longrightarrow \infty .
$$

Hence, $\mathfrak{T}$ is continuous.

Step 2: now, we are going to prove that the operator $\mathfrak{T}$ is bounded in set $\mathbb{X}$. For this, we show that, for any $\stackrel{p}{p}>0$, there exists $\stackrel{\circ}{q}>0$ such that, for each

$$
\mathrm{u} \in \mathfrak{Q}=\left\{\mathrm{u} \in \mathbb{X}:\|\mathrm{u}\|_{\mathbb{X}} \leq \stackrel{\circ}{p}\right\}
$$

$$
\left|\mathbf{z}_{n}(\sigma)-\mathbf{z}(\sigma)\right| \leq \frac{\mathfrak{Q}_{\phi}}{1-\mathfrak{Q}_{\phi}^{*}}\left|\mathrm{u}_{n}(\sigma)-\mathrm{u}(\sigma)\right| .
$$

Since we supposed that $\mathrm{u}_{n} \longrightarrow \mathrm{u}, \mathbf{z}_{n} \longrightarrow \mathrm{z}$ as $n \longrightarrow \infty$ for each $\sigma \in J$. So, by Lebesgue dominated convergence theorem [36], (47) gives

$$
\|\mathfrak{I}(\mathrm{u})\|_{\mathbb{X}} \leq \stackrel{\circ}{q}
$$

From (45), for each $\sigma \in J$, we have

$$
\begin{aligned}
\mathfrak{T} u(\sigma)= & -\frac{(\ln \sigma)^{\alpha-1}}{\Delta \Gamma(\alpha)}\left[\int_{1}^{\zeta} \int_{1}^{2} \varphi(\sigma)(\ln \sigma)^{\alpha-1-\beta} \mathbf{z}(\eta) \frac{\mathrm{d} \eta}{\eta} \mathrm{d} \sigma-\int_{1}^{\zeta} \int_{1}^{\sigma} \varphi(\sigma)\left(\ln \frac{\sigma}{\eta}\right)^{\alpha-1-\beta} \mathbf{z}(\eta) \frac{\mathrm{d} \eta}{\eta} \mathrm{d} \sigma\right] \\
& -\frac{(\ln \sigma)^{\alpha-1} \Gamma(\alpha-\beta)}{\Delta \Gamma^{2}(\alpha)}\left[\int_{1}^{2} \int_{1}^{2}(\ln \sigma)^{\alpha-1} \mathbf{z}(\eta) \frac{\mathrm{d} \eta}{\eta} \mathrm{d} \mu(\sigma)-\int_{1}^{2} \int_{1}^{\sigma}\left(\ln \frac{\sigma}{\eta}\right)^{\alpha-1} \mathbf{z}(\eta) \frac{\mathrm{d} \eta}{\eta} \mathrm{d} \mu(\sigma)\right] \\
& -\frac{(\ln \sigma)^{\alpha-1}}{\Gamma(\alpha)} \int_{1}^{2} \mathbf{z}(\eta) \frac{\mathrm{d} \eta}{\eta}+\frac{1}{\Gamma(\alpha)} \int_{1}^{\sigma}\left(\ln \frac{\sigma}{\eta}\right)^{\alpha-1} \mathbf{z}(\eta) \frac{\mathrm{d} \eta}{\eta} .
\end{aligned}
$$

Now, by $\left(\mathbf{M}_{3}\right)$ and $(46)$, we have

$\|z\|_{\mathbb{X}} \leq \pi_{1}^{*}+\pi_{2}^{*} \stackrel{\circ}{p}+\pi_{3}^{*}\|z\|_{\mathbb{X}}$

$$
\begin{aligned}
|\mathrm{z}(\sigma)| & =|-\phi(\sigma, \mathrm{u}(\sigma), \mathrm{z}(\sigma))| \\
& \leq \pi_{1}(\sigma)+\pi_{2}(\sigma)|\mathrm{u}(\sigma)|+\pi_{3}(\sigma)|\mathrm{z}(\sigma)| .
\end{aligned}
$$

Taking $\sup _{\sigma>0}$, we get

$$
\leq \frac{\pi_{1}^{*}+\pi_{2}^{*} \stackrel{\circ}{p}}{1-\pi_{3}^{*}} .
$$

Thus, (55) becomes

$$
\begin{aligned}
\|\mathfrak{I} u\|_{\mathbb{X}} \leq & {\left[\frac{\pi_{1}^{*}+\pi_{2}^{*} \stackrel{\circ}{p}}{1-\pi_{3}^{*}}\right]\left[\frac { ( \operatorname { l n } 2 ) ^ { \alpha - 1 } } { \nabla \Gamma ( \alpha + 1 ) } \left(\alpha \mathfrak{E} \zeta(\ln 2) \sum_{k=0}^{\alpha-1-\beta}(-1)^{\alpha-1-\beta-k} \frac{(\alpha-1-\beta) !}{k !}(\ln \zeta)^{k}\right.\right.} \\
& +\frac{\alpha \mathfrak{F} \zeta}{\alpha-\beta} \sum_{k=0}^{\alpha-\beta}(-1)^{\alpha-\beta-k} \frac{(\alpha-\beta) !}{k !}(\ln \zeta)^{k} \\
& \left.\left.+\mathfrak{P}(\ln \rho)^{\alpha-1} \Gamma(\alpha-\beta)(\alpha(\ln 2)+(\ln \rho))+\nabla(\alpha(\ln 2)+(\ln 2))\right)\right] \\
\leq & \frac{\pi_{1}^{*}+\pi_{2}^{*} \stackrel{\circ}{p}}{1-\pi_{3}^{*}} \mathscr{Y}=\stackrel{\circ}{q} .
\end{aligned}
$$


Hence, $\mathfrak{I}(\mathfrak{Q})$ is uniformly bounded.

Step 3: now, to show that the operator $\mathfrak{I}$ is equicontinuous in $\mathbb{X}$. For this, let $\sigma_{1}, \sigma_{2} \in J$ with $\sigma_{1}>\sigma_{2}$, since $\mathfrak{Q}$ is a bounded set in $\mathbb{X}$, and let $u \in \mathfrak{Q}$. Then,

$$
\begin{aligned}
& \left|\mathfrak{I}(\mathrm{u})\left(\sigma_{1}\right)-\mathfrak{T}(\mathrm{u})\left(\sigma_{2}\right)\right|=\mid-\frac{1}{\Delta \Gamma(\alpha)}\left[\left(\ln \sigma_{1}\right)^{\alpha-1} \int_{1}^{\zeta} \int_{1}^{2} \varphi\left(\sigma_{1}\right)\left(\ln \sigma_{1}\right)^{\alpha-1-\beta} \mathbf{z}(\eta) \frac{\mathrm{d} \eta}{\eta} \mathrm{d} \sigma_{1}\right. \\
& -\left(\ln \sigma_{2}\right)^{\alpha-1} \int_{1}^{\zeta} \int_{1}^{2} \varphi\left(\sigma_{2}\right)\left(\ln \sigma_{2}\right)^{\alpha-1-\beta} \mathbf{z}(\eta) \frac{\mathrm{d} \eta}{\eta} \mathrm{d} \sigma_{2} \\
& -\left(\ln \sigma_{1}\right)^{\alpha-1} \int_{1}^{\zeta} \int_{1}^{\sigma_{1}} \varphi\left(\sigma_{1}\right)\left(\ln \frac{\sigma_{1}}{\eta}\right)^{\alpha-1-\beta} \mathbf{z}(\eta) \frac{\mathrm{d} \eta}{\eta} \mathrm{d} \sigma_{1} \\
& \left.+\left(\ln \sigma_{2}\right)^{\alpha-1} \int_{1}^{\zeta} \int_{1}^{\sigma_{2}} \varphi\left(\sigma_{2}\right)\left(\ln \frac{\sigma_{2}}{\eta}\right)^{\alpha-1-\beta} \mathbf{z}(\eta) \frac{\mathrm{d} \eta}{\eta} \mathrm{d} \sigma_{2}\right] \\
& -\frac{\Gamma(\alpha-\beta)}{\Delta \Gamma^{2}(\alpha)}\left[\left(\ln \sigma_{1}\right)^{\alpha-1} \int_{1}^{2} \int_{1}^{2}\left(\ln \sigma_{1}\right)^{\alpha-1} \mathbf{z}(\eta) \frac{\mathrm{d} \eta}{\eta} \mathrm{d} \mu\left(\sigma_{1}\right)\right. \\
& -\left(\ln \sigma_{2}\right)^{\alpha-1} \int_{1}^{2} \int_{1}^{2}\left(\ln \sigma_{2}\right)^{\alpha-1} \mathbf{z}(\eta) \frac{\mathrm{d} \eta}{\eta} \mathrm{d} \mu\left(\sigma_{2}\right) \\
& -\left(\ln \sigma_{1}\right)^{\alpha-1} \int_{1}^{2} \int_{1}^{\sigma_{1}}\left(\ln \frac{\sigma_{1}}{\eta}\right)^{\alpha-1} \mathbf{z}(\eta) \frac{\mathrm{d} \eta}{\eta} \mathrm{d} \mu\left(\sigma_{1}\right) \\
& \left.+\left(\ln \sigma_{2}\right)^{\alpha-1} \int_{1}^{2} \int_{1}^{\sigma_{2}}\left(\ln \frac{\sigma_{2}}{\eta}\right)^{\alpha-1} \mathrm{z}(\eta) \frac{\mathrm{d} \eta}{\eta} \mathrm{d} \mu\left(\sigma_{2}\right)\right] \\
& -\frac{\left(\ln \sigma_{1}\right)^{\alpha-1}-\left(\ln \sigma_{2}\right)^{\alpha-1}}{\Gamma(\alpha)} \int_{1}^{2} \mathbf{z}(\eta) \frac{\mathrm{d} \eta}{\eta}+\frac{1}{\Gamma(\alpha)} \int_{\sigma_{2}}^{\sigma_{1}}\left(\ln \frac{\sigma_{1}}{\eta}\right)^{\alpha-1} \mathbf{z}(\eta) \frac{\mathrm{d} \eta}{\eta} \\
& -\frac{1}{\Gamma(\alpha)} \int_{1}^{\sigma_{2}}\left(\left(\ln \frac{\sigma_{2}}{\eta}\right)^{\alpha-1}-\left(\ln \frac{\sigma_{1}}{\eta}\right)^{\alpha-1}\right) z(\eta) \frac{\mathrm{d} \eta}{\eta} \mid \\
& \leq\left[\frac { ( ( \operatorname { l n } \sigma _ { 1 } ) ^ { \alpha - 1 } - ( \operatorname { l n } \sigma _ { 2 } ) ^ { \alpha - 1 } ) } { \nabla \Gamma ( \alpha + 1 ) } \left(\alpha \mathfrak{5} \zeta(\ln 2) \sum_{k=0}^{\alpha-1-\beta}(-1)^{\alpha-1-\beta-k} \frac{(\alpha-1-\beta) !}{k !}(\ln \zeta)^{k}\right.\right. \\
& +\frac{\alpha \mathfrak{E} \zeta}{\alpha-\beta} \sum_{k=0}^{\alpha-\beta}(-1)^{\alpha-\beta-k} \frac{(\alpha-\beta) !}{k !}(\ln \zeta)^{k} \\
& \left.+\mathfrak{P}(\ln \rho)^{\alpha-1} \Gamma(\alpha-\beta)(\alpha(\ln 2)+(\ln \rho))+\nabla \alpha(\ln 2)\right) \\
& \left.+\frac{\left(\left(\ln \sigma_{1}\right)^{\alpha}-\left(\ln \sigma_{2}\right)^{\alpha}\right)}{\Gamma(\alpha+1)}\right]\left[\frac{\pi_{1}^{*}+\pi_{2}^{*} \stackrel{\circ}{p}}{1-\pi_{3}^{*}}\right] .
\end{aligned}
$$

The right-hand side of (59) approaches to zero as $\sigma_{1} \longrightarrow \sigma_{2}$. Hence, $\mathfrak{I}(\mathfrak{Q})$ is equicontinuous. As a consequence of Step 1 to 3 , the operator $\mathfrak{I}$ is completely continuous. Therefore, in view of the Arzelà-Ascoli theorem, the operator $\mathfrak{T}$ is compact.
Theorem 6. Let the hypotheses $\left(\mathbf{M}_{3}\right)$ and $\left(\mathbf{M}_{5}\right)$ hold, and if $\mathfrak{N}<1$, the given problem (5) has at least one solution in $\mathbb{X}$.

Proof. For the proof of this theorem, we are considering a set $\mathscr{B} \subset \mathbb{X}$ which is defined in the following form: 


$$
\mathscr{B}=\{\mathrm{u} \in \mathbb{X} \mid \mathrm{u}=\delta \mathfrak{T} \mathrm{u}, \quad 0<\delta<1\} .
$$

We have to show that the set $\mathscr{B}$ is bounded. Let $\mathrm{u} \in \mathbb{X}$ such that

$$
\mathrm{u}(\sigma)=\delta \mathfrak{T} u(\sigma), \quad \text { where } 0<\delta<1 .
$$

Then, for each $\sigma \in J$, we have

$$
\begin{aligned}
& |\mathrm{u}(\sigma)|=\mid-\delta\left(\frac{(\ln \sigma)^{\alpha-1}}{\Delta \Gamma(\alpha)} \int_{1}^{\zeta} \int_{1}^{2} \varphi(\sigma)(\ln \sigma)^{\alpha-1-\beta} \mathrm{z}(\eta) \frac{\mathrm{d} \eta}{\eta} \mathrm{d} \sigma\right. \\
& -\frac{(\ln \sigma)^{\alpha-1}}{\Delta \Gamma(\alpha)} \int_{1}^{\zeta} \int_{1}^{\sigma} \varphi(\sigma)\left(\ln \frac{\sigma}{\eta}\right)^{\alpha-1-\beta} \mathrm{z}(\eta) \frac{\mathrm{d} \eta}{\eta} \mathrm{d} \sigma \\
& +\frac{(\ln \sigma)^{\alpha-1} \Gamma(\alpha-\beta)}{\Delta \Gamma^{2}(\alpha)} \int_{1}^{2} \int_{1}^{2}(\ln \sigma)^{\alpha-1} \mathrm{z}(\eta) \frac{\mathrm{d} \eta}{\eta} \mathrm{d} \mu(\sigma) \\
& -\frac{(\ln \sigma)^{\alpha-1} \Gamma(\alpha-\beta)}{\Delta \Gamma^{2}(\alpha)} \int_{1}^{2} \int_{1}^{\sigma}\left(\ln \frac{\sigma}{\eta}\right)^{\alpha-1} \mathrm{z}(\eta) \frac{\mathrm{d} \eta}{\eta} \mathrm{d} \mu(\sigma) \\
& \left.+\frac{(\ln \sigma)^{\alpha-1}}{\Gamma(\alpha)} \int_{1}^{2} \mathrm{z}(\eta) \frac{\mathrm{d} \eta}{\eta}-\frac{1}{\Gamma(\alpha)} \int_{1}^{\sigma}\left(\ln \frac{\sigma}{\eta}\right)^{\alpha-1} \mathrm{z}(\eta) \frac{\mathrm{d} \eta}{\eta}\right) \mid \\
& \leq \frac{(\ln 2)^{\alpha-1}}{\nabla \Gamma(\alpha+1)}|\mathbf{z}(\eta)|\left(\alpha \mathfrak{s} \zeta(\ln 2) \sum_{k=0}^{\alpha-1-\beta}(-1)^{\alpha-1-\beta-k} \frac{(\alpha-1-\beta) !}{k !}(\ln \zeta)^{k}\right. \\
& +\frac{\alpha \mathfrak{E} \zeta}{\alpha-\beta} \sum_{k=0}^{\alpha-\beta}(-1)^{\alpha-\beta-k} \frac{(\alpha-\beta) !}{k !}(\ln \zeta)^{k} \\
& \left.+\mathfrak{P}(\ln \rho)^{\alpha-1} \Gamma(\alpha-\beta)(\alpha(\ln 2)+(\ln \rho))+\nabla(\alpha(\ln 2)+(\ln 2))\right) . \\
& |\mathbf{z}(\sigma)| \leq \frac{\pi_{1}(\sigma)+\pi_{2}(\sigma)|\mathrm{u}(\sigma)|}{1-\pi_{3}(\sigma)} .
\end{aligned}
$$

Now, by $\left(\mathbf{M}_{3}\right)$, we have

$$
\begin{aligned}
|\mathbf{z}(\sigma)| & =|-\phi(\sigma, \mathrm{u}(\sigma), \mathrm{z}(\sigma))| \\
& \leq \pi_{1}(\sigma)+\pi_{2}(\sigma)|\mathrm{u}(\sigma)|+\pi_{3}(\sigma)|\mathrm{z}(\sigma)| .
\end{aligned}
$$

So, we get

$$
\begin{aligned}
\|\mathrm{u}\|_{\mathbb{X}} \leq & \frac{(\ln 2)^{\alpha-1}}{\nabla \Gamma(\alpha+1)}\left(\frac{\pi_{1}^{*}+\pi_{2}^{*}\|\mathrm{u}\|_{\mathbb{X}}}{1-\pi_{3}^{*}}\right)\left(\alpha \mathfrak{\xi} \zeta(\ln 2) \sum_{k=0}^{\alpha-1-\beta}(-1)^{\alpha-1-\beta-k} \frac{(\alpha-1-\beta) !}{k !}(\ln \zeta)^{k}\right. \\
& +\frac{\alpha \mathfrak{E} \zeta}{\alpha-\beta} \sum_{k=0}^{\alpha-\beta}(-1)^{\alpha-\beta-k} \frac{(\alpha-\beta) !}{k !}(\ln \zeta)^{k} \\
& \left.+\mathfrak{P}(\ln \rho)^{\alpha-1} \Gamma(\alpha-\beta)(\alpha(\ln 2)+(\ln \rho))+\nabla(\alpha(\ln 2)+(\ln 2))\right)
\end{aligned}
$$


For simplicity, let

$$
\begin{aligned}
\mathfrak{M}= & \frac{\pi_{1}^{*}(\ln 2)^{\alpha-1}}{\nabla\left(1-\pi_{3}^{*}\right) \Gamma(\alpha+1)}\left(\alpha \mathfrak{S} \zeta(\ln 2) \sum_{k=0}^{\alpha-1-\beta}(-1)^{\alpha-1-\beta-k} \frac{(\alpha-1-\beta) !}{k !}(\ln \zeta)^{k}\right. \\
& +\frac{\alpha \mathfrak{S} \zeta}{\alpha-\beta} \sum_{k=0}^{\alpha-\beta}(-1)^{\alpha-\beta-k} \frac{(\alpha-\beta) !}{k !}(\ln \zeta)^{k} \\
& \left.+\mathfrak{P}(\ln \rho)^{\alpha-1} \Gamma(\alpha-\beta)(\alpha(\ln 2)+(\ln \rho))+\nabla(\alpha(\ln 2)+(\ln 2))\right) \\
\mathfrak{N}= & \frac{\pi_{2}^{*}(\ln 2)^{\alpha-1}}{\nabla\left(1-\pi_{3}^{*}\right) \Gamma(\alpha+1)}\left(\alpha \mathfrak{S} \zeta(\ln 2) \sum_{k=0}^{\alpha-1-\beta}(-1)^{\alpha-1-\beta-k} \frac{(\alpha-1-\beta) !}{k !}(\ln \zeta)^{k}\right. \\
& +\frac{\alpha \mathfrak{E} \zeta}{\alpha-\beta} \sum_{k=0}^{\alpha-\beta}(-1)^{\alpha-\beta-k} \frac{(\alpha-\beta) !}{k !}(\ln \zeta)^{k} \\
& \left.+\mathfrak{P}(\ln \rho)^{\alpha-1} \Gamma(\alpha-\beta)(\alpha(\ln 2)+(\ln \rho))+\nabla(\alpha(\ln 2)+(\ln 2))\right) .
\end{aligned}
$$

So, (65) becomes

$$
\|\mathrm{u}\|_{\mathbb{X}} \leq \mathfrak{M}+\mathfrak{N}\|\mathrm{u}\|_{\mathbb{X}}
$$

which implies

$$
\|\mathrm{u}\|_{\mathbb{X}} \leq \frac{\mathfrak{M}}{1-\mathfrak{N}}
$$

This shows that the set $\mathscr{B}$ is bounded. So, by Theorems 3 and 5, we get that the operator $\mathfrak{I}$ has at least one fixed point. Therefore, the given problem (5) has at least one solution in $\mathbb{X}$.
Theorem 7. Suppose that the hypothesis $\left(\mathbf{M}_{2}\right),\left(\mathbf{M}_{4}\right)$, and $\left(\mathbf{M}_{5}\right)$ hold. Then, the given problem (5) has a unique solution in $\mathbb{X}$ if

$$
\frac{\mathfrak{Q}_{\phi}}{1-\mathfrak{Q}_{\phi}^{*}} \mathscr{y}<1 .
$$

Proof. We shall use the Banach contraction principle to prove that the operator $\mathfrak{I}$ has a unique fixed point, which will be the unique solution of the given system (5), by considering the operator $\mathfrak{T}: \mathbb{X} \longrightarrow \mathbb{X}$ defined in (45).

Let $\mathrm{u}, \overline{\mathrm{u}}$ be the solution of (5), and for $\sigma \in J$, we have

$$
\begin{aligned}
|\mathfrak{T}(\mathrm{u})(\sigma)-\mathfrak{T}(\overline{\mathrm{u}})(\sigma)| \leq & \frac{(\ln \sigma)^{\alpha-1}}{|\Delta| \Gamma(\alpha)} \int_{1}^{\zeta} \int_{1}^{2}|\varphi(\sigma)|(\ln \sigma)^{\alpha-1-\beta}|\mathbf{z}(\eta)-\overline{\mathbf{z}}(\eta)| \frac{\mathrm{d} \eta}{\eta} \mathrm{d} \sigma \\
& +\frac{(\ln \sigma)^{\alpha-1}}{|\Delta| \Gamma(\alpha)} \int_{1}^{\zeta} \int_{1}^{\sigma}|\varphi(\sigma)|\left|\left(\ln \frac{\sigma}{\eta}\right)^{\alpha-1-\beta}\right||\mathbf{z}(\eta)-\overline{\mathbf{z}}(\eta)| \frac{\mathrm{d} \eta}{\eta} \mathrm{d} \sigma \\
& +\frac{(\ln \sigma)^{\alpha-1} \Gamma(\alpha-\beta)}{|\Delta| \Gamma^{2}(\alpha)} \int_{1}^{2} \int_{1}^{2}(\ln \sigma)^{\alpha-1}|\mathbf{z}(\eta)-\overline{\mathbf{z}}(\eta)| \frac{\mathrm{d} \eta}{\eta} \mathrm{d} \mu(\sigma) \\
& +\frac{(\ln \sigma)^{\alpha-1} \Gamma(\alpha-\beta)}{|\Delta| \Gamma^{2}(\alpha)} \int_{1}^{2} \int_{1}^{\sigma}\left|\left(\ln \frac{\sigma}{\eta}\right)^{\alpha-1}\right||\mathbf{z}(\eta)-\overline{\mathbf{z}}(\eta)| \frac{\mathrm{d} \eta}{\eta} \mathrm{d} \mu(\sigma) \\
& +\frac{(\ln \sigma)^{\alpha-1}}{\Gamma(\alpha)} \int_{1}^{2}|\mathbf{z}(\eta)-\overline{\mathbf{z}}(\eta)| \frac{\mathrm{d} \eta}{\eta}+\frac{1}{\Gamma(\alpha)} \int_{1}^{\sigma}\left|\left(\ln \frac{\sigma}{\eta}\right)^{\alpha-1}\right||\mathbf{z}(\eta)-\overline{\mathbf{z}}(\eta)| \frac{\mathrm{d} \eta}{\eta},
\end{aligned}
$$


where $z, \bar{z} \in C(J, \mathbb{R})$ such that

$$
\begin{aligned}
& \mathbf{z}(\sigma)=-\phi(\sigma, \mathrm{u}(\sigma), \mathbf{z}(\sigma)), \\
& \overline{\mathbf{z}}(\sigma)=-\phi(\sigma, \overline{\mathrm{u}}(\sigma), \overline{\mathbf{z}}(\sigma)) .
\end{aligned}
$$

Now, by $\left(\mathbf{M}_{4}\right)$, we have

$$
\begin{aligned}
|\mathbf{z}(\sigma)-\overline{\mathbf{z}}(\sigma)| & =|-(\phi(\sigma, \mathrm{u}(\sigma), \mathbf{z}(\sigma))-\phi(\sigma, \overline{\mathrm{u}}(\sigma), \overline{\mathbf{z}}(\sigma)))| \\
& \leq \mathfrak{Q}_{\phi}|\mathrm{u}-\overline{\mathrm{u}}|+\mathbf{\Omega}_{\phi}^{*}|\mathbf{z}-\overline{\mathbf{z}}|,
\end{aligned}
$$

which implies

$$
|\mathbf{z}(\sigma)-\overline{\mathbf{z}}(\sigma)| \leq \frac{\mathfrak{Q}_{\phi}}{1-\mathfrak{\Omega}_{\phi}^{*}}|\mathrm{u}-\overline{\mathrm{u}}| .
$$

So, (70) becomes

$$
\begin{aligned}
|\mathfrak{T}(\mathrm{u})(\sigma)-\mathfrak{T}(\overline{\mathrm{u}})(\sigma)| \leq & \frac{\mathfrak{Q}_{\phi}}{1-\mathfrak{Q}_{\phi}^{*}}\left[\frac{(\ln \sigma)^{\alpha-1}}{|\Delta| \Gamma(\alpha)} \int_{1}^{\zeta} \int_{1}^{2}|\varphi(\sigma)|(\ln \sigma)^{\alpha-1-\beta}|\mathrm{u}(\eta)-\overline{\mathrm{u}}(\eta)| \frac{\mathrm{d} \eta}{\eta} \mathrm{d} \sigma\right. \\
& +\frac{(\ln \sigma)^{\alpha-1}}{|\Delta| \Gamma(\alpha)} \int_{1}^{\zeta} \int_{1}^{\sigma}|\varphi(\sigma)|\left(\ln \frac{\sigma}{\eta}\right)^{\alpha-1-\beta}|| \mathrm{u}(\eta)-\overline{\mathrm{u}}(\eta) \mid \frac{\mathrm{d} \eta}{\eta} \mathrm{d} \sigma \\
& +\frac{(\ln \sigma)^{\alpha-1} \Gamma(\alpha-\beta)}{|\Delta| \Gamma^{2}(\alpha)} \int_{1}^{2} \int_{1}^{2}(\ln \sigma)^{\alpha-1}|\mathrm{u}(\eta)-\overline{\mathrm{u}}(\eta)| \frac{\mathrm{d} \eta}{\eta} \mathrm{d} \mu(\sigma) \\
& +\frac{(\ln \sigma)^{\alpha-1} \Gamma(\alpha-\beta)}{|\Delta| \Gamma^{2}(\alpha)} \int_{1}^{2} \int_{1}^{\sigma}\left|\left(\ln \frac{\sigma}{\eta}\right)^{\alpha-1}\right||\mathrm{u}(\eta)-\overline{\mathrm{u}}(\eta)| \frac{\mathrm{d} \eta}{\eta} \mathrm{d} \mu(\sigma) \\
& \left.+\frac{(\ln \sigma)^{\alpha-1}}{\Gamma(\alpha)} \int_{1}^{2}|\mathrm{u}(\eta)-\overline{\mathrm{u}}(\eta)| \frac{\mathrm{d} \eta}{\eta}+\frac{1}{\Gamma(\alpha)} \int_{1}^{\sigma}\left|\left(\ln \frac{\sigma}{\eta}\right)^{\alpha-1}\right||\mathrm{u}(\eta)-\overline{\mathrm{u}}(\eta)| \frac{\mathrm{d} \eta}{\eta}\right] .
\end{aligned}
$$

Using $\left(\mathbf{M}_{5}\right)$ and taking sup $\operatorname{s>1}_{\sigma}$ on both sides, we get

$$
\begin{aligned}
\|\mathfrak{T}(\mathrm{u})-\mathfrak{T}(\overline{\mathrm{u}})\|_{\mathbb{X}} \leq & \frac{\mathfrak{Q}_{\phi}}{1-\mathfrak{\Omega}_{\phi}^{*}}\left[\frac { ( \operatorname { l n } 2 ) ^ { \alpha - 1 } } { \nabla \Gamma ( \alpha + 1 ) } \left(\alpha \mathfrak{F} \zeta(\ln 2) \sum_{k=0}^{\alpha-1-\beta}(-1)^{\alpha-1-\beta-k} \frac{(\alpha-1-\beta) !}{k !}(\ln \zeta)^{k}\right.\right. \\
& +\frac{\alpha \mathfrak{S} \zeta}{\alpha-\beta} \sum_{k=0}^{\alpha-\beta}(-1)^{\alpha-\beta-k} \frac{(\alpha-\beta) !}{k !}(\ln \zeta)^{k} \\
& \left.\left.+\mathfrak{P}(\ln \rho)^{\alpha-1} \Gamma(\alpha-\beta)(\alpha(\ln 2)+(\ln \rho))+\nabla(\alpha(\ln 2)+(\ln 2))\right)\right]\|\mathrm{u}-\overline{\mathrm{u}}\|_{\mathbb{X}} .
\end{aligned}
$$

This implies that

$$
\|\mathfrak{I}(\mathrm{u})-\mathfrak{T}(\overline{\mathrm{u}})\|_{\mathbb{X}} \leq \frac{\mathfrak{Q}_{\phi}}{1-\mathfrak{\Omega}_{\phi}^{*}} \mathscr{Y}\|\mathrm{u}-\overline{\mathrm{u}}\|_{\mathbb{X}} .
$$

Hence, the operator $\mathfrak{T}$ is a contraction. Thus, by the Banach contraction principle, we get that $\mathfrak{I}$ has a unique fixed point, which is a unique solution of the given problem (5).

3.2. Existence and Uniqueness Solution for System (7). In this section, we show the existence and uniqueness of the solution of the system (7). First, we have the following: 
Lemma 5. The system

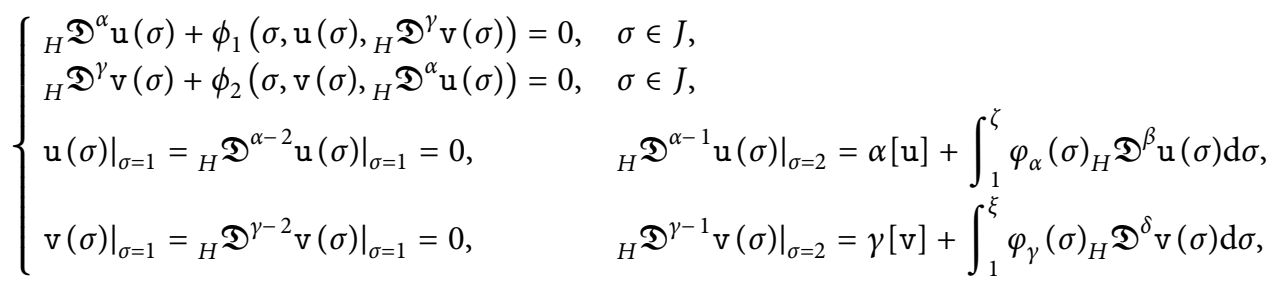

has a solution $(u, v)$ if and only if

where

$$
\left\{\begin{array}{l}
\mathrm{u}(\sigma)=\int_{1}^{2} \mathbb{G}_{\alpha}(\sigma, \eta) \phi_{1}\left(\eta, \mathrm{u}(\eta),{ }_{H} \mathfrak{D}^{\gamma} \mathrm{v}(\eta)\right) \frac{\mathrm{d} \eta}{\eta}, \\
\mathrm{v}(\sigma)=\int_{1}^{2} \mathbb{G}_{\gamma}(\sigma, \eta) \phi_{2}\left(\eta, \mathrm{v}(\eta),{ }_{H} \mathfrak{D}^{\alpha} \mathrm{u}(\eta)\right) \frac{\mathrm{d} \eta}{\eta},
\end{array}\right.
$$

$$
\begin{aligned}
& \mathbb{G}_{\alpha}(\sigma, \eta)=\mathbb{G}_{1}(\sigma, \eta)+\mathbb{G}_{2}(\sigma, \eta)+\mathbb{G}_{3}(\sigma, \eta), \\
& \mathbb{G}_{\gamma}(\sigma, \eta)=\mathbb{G}_{4}(\sigma, \eta)+\mathbb{G}_{5}(\sigma, \eta)+\mathbb{G}_{6}(\sigma, \eta) \text {, } \\
& \mathbb{G}_{1}(\sigma, \eta)= \begin{cases}\frac{(\ln \sigma)^{\alpha-1}-(\ln (\sigma / \eta))^{\alpha-1}}{\Gamma(\alpha)}, & 1 \leq \eta \leq \sigma \leq 2, \\
\frac{(\ln \sigma)^{\alpha-1}}{\Gamma(\alpha)}, & 1 \leq \sigma \leq \eta \leq 2,\end{cases} \\
& \mathbb{G}_{2}(\sigma, \eta)=\frac{(\ln \sigma)^{\alpha-1}}{\Delta_{\alpha}} \int_{1}^{\zeta} \varphi_{\alpha}(\sigma) \mathbb{Q}_{1}(\sigma, \eta) \mathrm{d} \sigma, \\
& \mathbb{G}_{3}(\sigma, \eta)=\frac{\Gamma(\alpha-\beta)(\ln \sigma)^{\alpha-1}}{\Delta_{\alpha} \Gamma(\alpha)} \int_{1}^{2} \mathbb{G}_{1}(\sigma, \eta) \mathrm{d} \mu(\sigma), \\
& \mathbb{G}_{4}(\sigma, \eta)= \begin{cases}\frac{(\ln \sigma)^{\gamma-1}-(\ln (\sigma / \eta))^{\gamma-1}}{\Gamma(\gamma)}, & 1 \leq \eta \leq \sigma \leq 2, \\
\frac{(\ln \sigma)^{\gamma-1}}{\Gamma(\gamma)}, & 1 \leq \sigma \leq \eta \leq 2,\end{cases}
\end{aligned}
$$$$
\mathbb{G}_{5}(\sigma, \eta)=\frac{(\ln \sigma)^{\gamma-1}}{\Delta_{\gamma}} \int_{1}^{\xi} \varphi_{\gamma}(\sigma) \mathbb{Q}_{1}(\sigma, \eta) \mathrm{d} \sigma,
$$$$
\mathbb{G}_{6}(\sigma, \eta)=\frac{\Gamma(\gamma-\delta)(\ln \sigma)^{\gamma-1}}{\Delta_{\gamma} \Gamma(\gamma)} \int_{1}^{2} \mathbb{G}_{4}(\sigma, \eta) \mathrm{d} \mu(\sigma),
$$$$
\mathbb{Q}_{1}(\sigma, \eta)= \begin{cases}\frac{(\ln \sigma)^{\alpha-1-\beta}-(\ln (\sigma / \eta))^{\alpha-1-\beta}}{\Gamma(\alpha)}, & 1 \leq \eta \leq \sigma \leq 2, \\ \frac{(\ln \sigma)^{\alpha-1-\beta}}{\Gamma(\alpha)}, & 1 \leq \sigma \leq \eta \leq 2,\end{cases}
$$$$
\mathbb{Q}_{2}(\sigma, \eta)= \begin{cases}\frac{(\ln \sigma)^{\gamma-1-\delta}-(\ln (\sigma / \eta))^{\gamma-1-\delta}}{\Gamma(\gamma)}, & 1 \leq \eta \leq \sigma \leq 2, \\ \frac{(\ln \sigma)^{\gamma-1-\delta}}{\Gamma(\gamma)}, & 1 \leq \sigma \leq \eta \leq 2 .\end{cases}
$$ 
Proof. The proof is similar to that given in Lemma 3 and, hence, is not included here.We use the following notations for convenience:

$$
\begin{aligned}
& \mathrm{y}(\sigma):=-\phi_{1}\left(\sigma, \mathrm{u}(\sigma),{ }_{H} \mathfrak{D}^{\gamma} \mathrm{v}(\sigma)\right)=-\phi_{1}(\sigma, \mathrm{u}(\sigma), \mathrm{z}(\sigma)), \\
& \mathrm{z}(\sigma):=-\phi_{2}\left(\sigma, \mathrm{v}(\sigma){ }_{H} \mathfrak{D}^{\alpha} \mathrm{u}(\sigma)\right)=-\phi_{2}(\sigma, \mathrm{v}(\sigma), \mathrm{y}(\sigma)) .
\end{aligned}
$$

Hence, for $\sigma \in J$, (78) becomes

$$
\left\{\begin{array}{l}
\mathrm{u}(\sigma)=\int_{1}^{2} \mathbb{G}_{\alpha}(\sigma, \eta) \mathrm{y}(\eta) \frac{\mathrm{d} \eta}{\eta}, \\
\mathrm{v}(\sigma)=\int_{1}^{2} \mathbb{G}_{\gamma}(\sigma, \eta) \mathrm{z}(\eta) \frac{\mathrm{d} \eta}{\eta},
\end{array}\right.
$$

where $y, z \in \mathbb{X}$ satisfying the functional equation.

Lemma 6. Green's function $\mathbb{G}_{\alpha, \gamma}(\sigma, \eta)=\left(\mathbb{G}_{\alpha}(\sigma, \eta), \mathbb{G}_{\gamma}\right.$ $(\sigma, \eta))$ of the system (7) have the following properties:

(1) $\mathbb{G}_{\alpha, \gamma}(\sigma, \eta)$ is continuous over $J \times J$

(2) $\max _{\sigma \in J} \int_{1}^{2}\left|\mathbb{G}_{\alpha}(\sigma, \eta)\right| d \eta \mid \quad \eta \leqslant \mathscr{Y}_{\alpha}$ and $\max _{\sigma \in J} \int_{1}^{2} \mid \mathbb{G}_{\gamma}$ $(\sigma, \eta) \mid d \eta / \eta \leqslant \mathscr{Y}_{\gamma}$,

where

$$
\begin{aligned}
\mathscr{Y}_{\alpha}= & \frac{(\ln 2)^{\alpha-1}}{\nabla_{\alpha} \Gamma(\alpha+1)}\left(\alpha \mathfrak{F}_{\alpha} \zeta(\ln 2) \sum_{k=0}^{\alpha-1-\beta}(-1)^{\alpha-1-\beta-k} \frac{(\alpha-1-\beta) !}{k !}(\ln \zeta)^{k}\right. \\
& +\frac{\alpha \mathfrak{F}_{\alpha} \zeta}{\alpha-\beta} \sum_{k=0}^{\alpha-\beta}(-1)^{\alpha-\beta-k} \frac{(\alpha-\beta) !}{k !}(\ln \zeta)^{k} \\
+ & \left.\mathfrak{P}(\ln \rho)^{\alpha-1} \Gamma(\alpha-\beta)(\alpha(\ln 2)+(\ln \rho))+\nabla_{\alpha}(\alpha(\ln 2)+(\ln 2))\right), \\
\mathscr{Y}_{\gamma}= & \frac{(\ln 2)^{\gamma-1}}{\nabla_{\gamma} \Gamma(\gamma+1)}\left(\gamma \mathfrak{F}_{\gamma} \xi(\ln 2) \sum_{k=0}^{\gamma-1-\delta}(-1)^{\gamma-1-\delta-k} \frac{(\gamma-1-\delta) !}{k !}(\ln \xi)^{k}\right. \\
& +\frac{\gamma\left(\mathfrak{F}_{\gamma} \xi\right.}{\gamma-\delta} \sum_{k=0}^{\gamma-\delta}(-1)^{\gamma-\delta-k} \frac{(\gamma-\delta) !}{k !}(\ln \xi)^{k} \\
\nabla_{\gamma}= & \Gamma(\gamma-\delta)+\mathfrak{F}_{\gamma} \xi \sum_{k=0}^{\gamma-1-\delta}(-1)^{\gamma-1-\delta-k(\gamma-1-\delta) !}(\ln \xi)^{k}+\frac{(\ln \rho)^{\gamma-1} \mathfrak{P} \Gamma(\gamma-\delta)}{k !} \Gamma(\gamma) \\
\nabla_{\alpha}= & \Gamma(\alpha-\beta)+\mathfrak{F}_{\alpha} \zeta \sum_{k=0}^{\alpha-1-\beta}(-1)^{\alpha-1-\beta-k} \frac{(\alpha-1-\beta) !}{k !}(\ln \zeta)^{k}+\frac{(\ln \rho)^{\alpha-1} \mathfrak{P} \Gamma(\alpha-\beta)}{\Gamma(\alpha)} \\
+ & \left.\mathfrak{P}(\ln \rho)^{\gamma-1} \Gamma(\gamma-\delta)(\gamma(\ln 2)+(\ln \rho))+\nabla_{\gamma}(\gamma(\ln 2)+(\ln 2))\right)
\end{aligned}
$$

Proof. (1) It is easy to prove that $\mathbb{G}_{\alpha, \gamma}(\sigma, \eta)$ is continuous, so we leave it.
(2) Since we know that Green's functions of the considered problem (7) is in the form 


$$
\begin{aligned}
\int_{1}^{2}\left|\mathbb{G}_{\alpha}(\sigma, \eta)\right| \frac{\mathrm{d} \eta}{\eta}= & \mid \frac{(\ln \sigma)^{\alpha-1}}{\Delta_{\alpha} \Gamma(\alpha)}\left[\int_{1}^{\zeta} \int_{1}^{2} \varphi_{\alpha}(\sigma)(\ln \sigma)^{\alpha-1-\beta} \frac{\mathrm{d} \eta}{\eta} \mathrm{d} \sigma-\int_{1}^{\zeta} \int_{1}^{\sigma} \varphi_{\alpha}(\sigma)\left(\ln \frac{\sigma}{\eta}\right)^{\alpha-1-\beta} \frac{\mathrm{d} \eta}{\eta} \mathrm{d} \sigma\right] \\
& +\frac{(\ln \sigma)^{\alpha-1} \Gamma(\alpha-\beta)}{\Delta_{\alpha} \Gamma^{2}(\alpha)}\left[\int_{1}^{2} \int_{1}^{2}(\ln \sigma)^{\alpha-1} \frac{\mathrm{d} \eta}{\eta} \mathrm{d} \mu(\sigma)-\int_{1}^{2} \int_{1}^{\sigma}\left(\ln \frac{\sigma}{\eta}\right)^{\alpha-1} \frac{\mathrm{d} \eta}{\eta} \mathrm{d} \mu(\sigma)\right] \\
& +\frac{(\ln \sigma)^{\alpha-1}}{\Gamma(\alpha)} \int_{1}^{2} \frac{\mathrm{d} \eta}{\eta}-\frac{1}{\Gamma(\alpha)} \int_{1}^{\sigma}\left(\ln \frac{\sigma}{\eta}\right)^{\alpha-1} \frac{\mathrm{d} \eta}{\eta} \mid \\
\leq & \frac{(\ln \sigma)^{\alpha-1}}{\left|\Delta_{\alpha}\right| \Gamma(\alpha)}\left[\int_{1}^{\zeta} \int_{1}^{2}\left|\varphi_{\alpha}(\sigma)\right|(\ln \sigma)^{\alpha-1-\beta} \frac{\mathrm{d} \eta}{\eta} \mathrm{d} \sigma+\int_{1}^{\zeta} \int_{1}^{\sigma}\left|\varphi_{\alpha}(\sigma)\right|\left(\ln \frac{\sigma}{\eta}\right)^{\alpha-1-\beta} \mid \frac{\mathrm{d} \eta}{\eta} \mathrm{d} \sigma\right] \\
& +\frac{(\ln \sigma)^{\alpha-1} \Gamma(\alpha-\beta)}{\left|\Delta_{\alpha}\right| \Gamma^{2}(\alpha)}\left[\int_{1}^{2} \int_{1}^{2}(\ln \sigma)^{\alpha-1} \frac{\mathrm{d} \eta}{\eta} \mathrm{d} \mu(\sigma)+\int_{1}^{2} \int_{1}^{\sigma}\left|\left(\ln \frac{\sigma}{\eta}\right)^{\alpha-1}\right| \frac{\mathrm{d} \eta}{\eta} \mathrm{d} \mu(\sigma)\right] \\
& +\frac{(\ln \sigma)^{\alpha-1}}{\Gamma(\alpha)} \int_{1}^{2} \frac{\mathrm{d} \eta}{\eta}+\frac{1}{\Gamma(\alpha)} \int_{1}^{\sigma}\left|\left(\ln \frac{\sigma}{\eta}\right)^{\alpha-1}\right| \frac{\mathrm{d} \eta}{\eta},
\end{aligned}
$$

using $\left(\mathbf{M}_{5}\right)$ and the mean value theorem [35] with $\rho \in[1,2]$ and $\mu(2)=\mathfrak{P}>0$, we get

$$
\begin{aligned}
\max _{\sigma \in J} \int_{1}^{2}\left|\mathbb{G}_{\alpha}(\sigma, \eta)\right| \frac{\mathrm{d} \eta}{\eta} \leq & \frac{(\ln 2)^{\alpha-1}}{\nabla_{\alpha} \Gamma(\alpha+1)}\left(\alpha \mathfrak{E} \zeta(\ln 2) \sum_{k=0}^{\alpha-1-\beta}(-1)^{\alpha-1-\beta-k} \frac{(\alpha-1-\beta) !}{k !}(\ln \zeta)^{k}\right. \\
& +\frac{\alpha \mathfrak{E} \zeta}{\alpha-\beta} \sum_{k=0}^{\alpha-\beta}(-1)^{\alpha-\beta-k} \frac{(\alpha-\beta) !}{k !}(\ln \zeta)^{k} \\
& \left.+\mathfrak{P}(\ln \rho)^{\alpha-1} \Gamma(\alpha-\beta)(\alpha(\ln 2)+(\ln \rho))+\nabla_{\alpha}(\alpha(\ln 2)+(\ln 2))\right)=\mathscr{Y}_{\alpha} .
\end{aligned}
$$

Similarly, we can obtain

$$
\begin{aligned}
\max _{\sigma \in J} \int_{1}^{2}\left|\mathbb{G}_{\gamma}(\sigma, \eta)\right| \frac{\mathrm{d} \eta}{\eta} \leq & \frac{(\ln 2)^{\gamma-1}}{\nabla_{\gamma} \Gamma(\gamma+1)}\left(\gamma \mathfrak{E} \xi(\ln 2) \sum_{k=0}^{\gamma-1-\delta}(-1)^{\gamma-1-\delta-k} \frac{(\gamma-1-\delta) !}{k !}(\ln \xi)^{k}\right. \\
& +\frac{\gamma \mathfrak{E} \xi}{\gamma-\delta} \sum_{k=0}^{\gamma-\delta}(-1)^{\gamma-\delta-k} \frac{(\gamma-\delta) !}{k !}(\ln \xi)^{k} \\
& \left.+\mathfrak{P}(\ln \rho)^{\gamma-1} \Gamma(\gamma-\delta)(\gamma(\ln 2)+(\ln \rho))+\nabla_{\gamma}(\gamma(\ln 2)+(\ln 2))\right)=\mathscr{Y}_{\gamma} .
\end{aligned}
$$


Hence, the proof of 2 is complete.

If $\mathrm{u}, \mathrm{v}$ are the solutions of the given system (7) and $\sigma \in J$; then,

$$
\begin{aligned}
\mathrm{u}(\sigma)= & \frac{(\ln \sigma)^{\alpha-1}}{\Delta_{\alpha} \Gamma(\alpha)} \int_{1}^{\zeta} \int_{1}^{2} \varphi_{\alpha}(\sigma)(\ln \sigma)^{\alpha-1-\beta} \phi_{1}(\eta, \mathrm{u}(\eta), \mathrm{z}(\eta)) \frac{\mathrm{d} \eta}{\eta} \mathrm{d} \sigma \\
& -\frac{(\ln \sigma)^{\alpha-1}}{\Delta_{\alpha} \Gamma(\alpha)} \int_{1}^{\zeta} \int_{1}^{\sigma} \varphi_{\alpha}(\sigma)\left(\ln \frac{\sigma}{\eta}\right)^{\alpha-1-\beta} \phi_{1}(\eta, \mathrm{u}(\eta), \mathrm{z}(\eta)) \frac{\mathrm{d} \eta}{\eta} \mathrm{d} \sigma \\
& +\frac{(\ln \sigma)^{\alpha-1} \Gamma(\alpha-\beta)}{\Delta_{\alpha} \Gamma^{2}(\alpha)} \int_{1}^{2} \int_{1}^{2}(\ln \sigma)^{\alpha-1} \phi_{1}(\eta, \mathrm{u}(\eta), \mathrm{z}(\eta)) \frac{\mathrm{d} \eta}{\eta} \mathrm{d} \mu(\sigma) \\
& -\frac{(\ln \sigma)^{\alpha-1} \Gamma(\alpha-\beta)}{\Delta_{\alpha} \Gamma^{2}(\alpha)} \int_{1}^{2} \int_{1}^{\sigma}\left(\ln \frac{\sigma}{\eta}\right)^{\alpha-1} \phi_{1}(\eta, \mathrm{u}(\eta), \mathrm{z}(\eta)) \frac{\mathrm{d} \eta}{\eta} \mathrm{d} \mu(\sigma) \\
& +\frac{(\ln \sigma)^{\alpha-1}}{\Gamma(\alpha)} \int_{1}^{2} \phi_{1}(\eta, \mathrm{u}(\eta), \mathrm{z}(\eta)) \frac{\mathrm{d} \eta}{\eta}-\frac{1}{\Gamma(\alpha)} \int_{1}^{\sigma}\left(\ln \frac{\sigma}{\eta}\right)^{\alpha-1} \phi_{1}(\eta, \mathrm{u}(\eta), \mathrm{z}(\eta)) \frac{\mathrm{d} \eta}{\eta}, \\
\mathrm{v}(\sigma)= & \frac{(\ln \sigma)^{\gamma-1}}{\Delta_{\gamma} \Gamma(\gamma)} \int_{1}^{\xi} \int_{1}^{2} \varphi_{\gamma}(\sigma)(\ln \sigma)^{\gamma-1-\delta} \phi_{2}(\eta, \mathrm{v}(\eta), \mathrm{y}(\eta)) \frac{\mathrm{d} \eta}{\eta} \mathrm{d} \sigma \\
& +\frac{(\ln \sigma)^{\gamma-1}}{\Gamma(\gamma)} \int_{1}^{2} \phi_{2}(\eta, \mathrm{v}(\eta), \mathrm{y}(\eta)) \frac{\mathrm{d} \eta}{\eta}-\frac{1}{\Gamma(\gamma)} \int_{1}^{\sigma}\left(\ln \frac{\sigma}{\eta}\right)^{\gamma-1} \phi(\eta, \mathrm{v}(\eta), \mathrm{y}(\eta)) \frac{\mathrm{d} \eta}{\eta} \\
& -\frac{(\ln \sigma)^{\gamma-1}}{\Delta_{\gamma} \Gamma(\gamma)} \int_{1}^{\xi} \int_{1}^{\sigma} \varphi_{\gamma}(\sigma)\left(\ln \frac{\sigma}{\eta}\right)^{\gamma-1-\delta} \phi_{2}(\eta, \mathrm{v}(\eta), \mathrm{y}(\eta)) \frac{\mathrm{d} \eta}{\eta} \mathrm{d} \sigma \\
& +\frac{(\ln \sigma)^{\gamma-1} \Gamma(\gamma-\delta)}{\Delta_{\gamma} \Gamma^{2}(\gamma)} \int_{1}^{2} \int_{1}^{2}(\ln \sigma)^{\gamma-1} \phi_{2}(\eta, \mathrm{v}(\eta), \mathrm{y}(\eta)) \frac{\mathrm{d} \eta}{\eta} \mathrm{d} \mu(\sigma) \\
& \frac{(\ln \sigma)^{\gamma-1} \Gamma(\gamma-\delta)}{\Delta_{\gamma} \Gamma^{2}(\gamma)} \int_{1}^{2} \int_{1}^{\sigma}\left(\ln \frac{\sigma}{\eta}\right)^{\gamma-1} \phi_{2}(\eta, \mathrm{v}(\eta), \mathrm{y}(\eta)) \frac{\mathrm{d} \eta}{\eta} \mathrm{d} \mu(\sigma)
\end{aligned}
$$

Now, we transform the given system (7) into a fixedpoint problem. Let an operator $\mathfrak{I}: \mathbb{X} \times \mathbb{X} \longrightarrow \mathbb{X} \times \mathbb{X}$ be defined as

$$
\mathfrak{I}(\mathrm{u}, \mathrm{v})(\sigma)=\left(\begin{array}{l}
\int_{1}^{2} \mathbb{G}_{\alpha}(\sigma, \eta) \phi_{1}(\eta, \mathrm{u}(\eta), \mathrm{z}(\eta)) \frac{\mathrm{d} \eta}{\eta} \\
\int_{1}^{2} \mathbb{G}_{\gamma}(\sigma, \eta) \phi_{2}(\eta, \mathrm{v}(\eta), \mathrm{y}(\eta)) \frac{\mathrm{d} \eta}{\eta}
\end{array}\right)=\left(\begin{array}{c}
\mathfrak{T}_{\alpha}(\mathrm{u}, \mathrm{z})(\sigma) \\
\mathfrak{T}_{\gamma}(\mathrm{v}, \mathrm{y})(\sigma)
\end{array}\right)
$$


Then, the solution of (7) coincides with the fixed point of

$\mathfrak{I}$, where

$$
\begin{aligned}
\mathfrak{T}_{\alpha}(\mathrm{u}, \mathrm{z})(\sigma)= & -\frac{(\ln \sigma)^{\alpha-1}}{\Delta_{\alpha} \Gamma(\alpha)}\left[\int_{1}^{\zeta} \int_{1}^{2} \varphi_{\alpha}(\sigma)(\ln \sigma)^{\alpha-1-\beta} \mathrm{y}(\eta) \frac{\mathrm{d} \eta}{\eta} \mathrm{d} \sigma-\int_{1}^{\zeta} \int_{1}^{\sigma} \varphi_{\alpha}(\sigma)\left(\ln \frac{\sigma}{\eta}\right)^{\alpha-1-\beta} \mathrm{y}(\eta) \frac{\mathrm{d} \eta}{\eta} \mathrm{d} \sigma\right] \\
& -\frac{(\ln \sigma)^{\alpha-1} \Gamma(\alpha-\beta)}{\Delta_{\alpha} \Gamma^{2}(\alpha)}\left[\int_{1}^{2} \int_{1}^{2}(\ln \sigma)^{\alpha-1} \mathrm{y}(\eta) \frac{\mathrm{d} \eta}{\eta} \mathrm{d} \mu(\sigma)-\int_{1}^{2} \int_{1}^{\sigma}\left(\ln \frac{\sigma}{\eta}\right)^{\alpha-1} \mathrm{y}(\eta) \frac{\mathrm{d} \eta}{\eta} \mathrm{d} \mu(\sigma)\right] \\
& -\frac{(\ln \sigma)^{\alpha-1}}{\Gamma(\alpha)} \int_{1}^{2} \mathrm{y}(\eta) \frac{\mathrm{d} \eta}{\eta}+\frac{1}{\Gamma(\alpha)} \int_{1}^{\sigma}\left(\ln \frac{\sigma}{\eta}\right)^{\alpha-1} \mathrm{y}(\eta) \frac{\mathrm{d} \eta}{\eta}, \\
\mathfrak{T}_{\gamma}(\mathrm{v}, \mathrm{y})(\sigma)= & -\frac{(\ln \sigma)^{\gamma-1}}{\Delta_{\gamma} \Gamma(\gamma)}\left[\int_{1}^{\xi} \int_{1}^{2} \varphi_{\gamma}(\sigma)(\ln \sigma)^{\gamma-1-\delta} \mathrm{z}(\eta) \frac{\mathrm{d} \eta}{\eta} \mathrm{d} \sigma-\int_{1}^{\xi} \int_{1}^{\sigma} \varphi_{\gamma}(\sigma)\left(\ln \frac{\sigma}{\eta}\right)^{\gamma-1-\delta} \mathrm{z}(\eta) \frac{\mathrm{d} \eta}{\eta} \mathrm{d} \sigma\right] \\
& -\frac{(\ln \sigma)^{\gamma-1} \Gamma(\gamma-\delta)}{\Delta_{\gamma} \Gamma^{2}(\gamma)}\left[\int_{1}^{2} \int_{1}^{2}(\ln \sigma)^{\gamma-1} \mathbf{z}(\eta) \frac{\mathrm{d} \eta}{\eta} \mathrm{d} \mu(\sigma)-\int_{1}^{2} \int_{1}^{\sigma}\left(\ln \frac{\sigma}{\eta}\right)^{\gamma-1} \mathbf{z}(\eta) \frac{\mathrm{d} \eta}{\eta} \mathrm{d} \mu(\sigma)\right] \\
& -\frac{(\ln \sigma)^{\gamma-1}}{\Gamma(\gamma)} \int_{1}^{2} \mathrm{z}(\eta) \frac{\mathrm{d} \eta}{\eta}+\frac{1}{\Gamma(\gamma)} \int_{1}^{\sigma}\left(\ln \frac{\sigma}{\eta}\right)^{\gamma-1} \mathbf{z}(\eta) \frac{\mathrm{d} \eta}{\eta} \cdot
\end{aligned}
$$

Theorem 8. Let $\phi_{1}, \phi_{2}: J \times \mathbb{R} \times \mathbb{R} \longrightarrow \mathbb{R}$ and $\left(\mathbf{M}_{9}\right),\left(\mathbf{M}_{11}\right)$ hold. Then, the operator $\mathfrak{I}: \mathscr{C} \longrightarrow \mathscr{C}$ defined in (87) is completely continuous.
Proof. In view of continuity of $\phi_{1}, \phi_{2}$ and $\mathbb{G}_{\alpha, \gamma}(\sigma, \eta), \mathfrak{T}$ is also continuous for all $(\mathrm{u}, z) \in \mathscr{C}$. Suppose $\mathscr{B} \subseteq \mathscr{C}$ is a bounded set. So, for every $u \in \mathscr{B}$, we have

$$
\begin{aligned}
\left|\mathfrak{I}_{\alpha}(\mathrm{u}, \mathrm{v})(\sigma)\right|= & -\frac{(\ln \sigma)^{\alpha-1}}{\Delta_{\alpha} \Gamma(\alpha)}\left[\int_{1}^{\zeta} \int_{1}^{2} \varphi_{\alpha}(\sigma)(\ln \sigma)^{\alpha-1-\beta} \mathrm{y}(\eta) \frac{\mathrm{d} \eta}{\eta} \mathrm{d} \sigma-\int_{1}^{\zeta} \int_{1}^{\sigma} \varphi_{\alpha}(\sigma)\left(\ln \frac{\sigma}{\eta}\right)^{\alpha-1-\beta} \mathrm{y}(\eta) \frac{\mathrm{d} \eta}{\eta} \mathrm{d} \sigma\right] \\
& -\frac{(\ln \sigma)^{\alpha-1} \Gamma(\alpha-\beta)}{\Delta_{\alpha} \Gamma^{2}(\alpha)}\left[\int_{1}^{2} \int_{1}^{2}(\ln \sigma)^{\alpha-1} \mathrm{y}(\eta) \frac{\mathrm{d} \eta}{\eta} \mathrm{d} \mu(\sigma)-\int_{1}^{2} \int_{1}^{\sigma}\left(\ln \frac{\sigma}{\eta}\right)^{\alpha-1} \mathrm{y}(\eta) \frac{\mathrm{d} \eta}{\eta} \mathrm{d} \mu(\sigma)\right] \\
& -\frac{(\ln \sigma)^{\alpha-1}}{\Gamma(\alpha)} \int_{1}^{2} \mathrm{y}(\eta) \frac{\mathrm{d} \eta}{\eta}+\frac{1}{\Gamma(\alpha)} \int_{1}^{\sigma}\left(\ln \frac{\sigma}{\eta}\right)^{\alpha-1} \mathrm{y}(\eta) \frac{\mathrm{d} \eta}{\eta} \mid \\
\leqslant & \frac{(\ln \sigma)^{\alpha-1}}{\left|\Delta_{\alpha}\right| \Gamma(\alpha)}\left[\int_{1}^{\zeta} \int_{1}^{2}\left|\varphi_{\alpha}(\sigma)\right|(\ln \sigma)^{\alpha-1-\beta}|\mathrm{y}(\eta)| \frac{\mathrm{d} \eta}{\eta} \mathrm{d} \sigma+\int_{1}^{\zeta} \int_{1}^{\sigma}\left|\varphi_{\alpha}(\sigma)\right|\left(\ln \frac{\sigma}{\eta}\right)^{\alpha-1-\beta}|\mathrm{y}(\eta)| \frac{\mathrm{d} \eta}{\eta} \mathrm{d} \sigma\right] \\
& +\frac{(\ln \sigma)^{\alpha-1} \Gamma(\alpha-\beta)}{\left|\Delta_{\alpha}\right| \Gamma^{2}(\alpha)}\left[\int_{1}^{2} \int_{1}^{2}(\ln \sigma)^{\alpha-1}|\mathrm{y}(\eta)| \frac{\mathrm{d} \eta}{\eta} \mathrm{d} \mu(\sigma)+\int_{1}^{2} \int_{1}^{\sigma}\left|\left(\ln \frac{\sigma}{\eta}\right)^{\alpha-1}\right||\mathrm{y}(\eta)| \frac{\mathrm{d} \eta}{\eta} \mathrm{d} \mu(\sigma)\right] \\
& +\frac{(\ln \sigma)^{\alpha-1}}{\Gamma(\alpha)} \int_{1}^{2}|\mathrm{y}(\eta)| \frac{\mathrm{d} \eta}{\eta}+\frac{1}{\Gamma(\alpha)} \int_{1}^{\sigma}\left|\left(\ln \frac{\sigma}{\eta}\right)^{\alpha-1}\right| \operatorname{ly}(\eta) \mid \frac{\mathrm{d} \eta}{\eta} .
\end{aligned}
$$


Now, by $\left(\mathbf{M}_{9}\right)$, we have

$$
\begin{aligned}
|\mathrm{y}(\sigma)| & =\left|-\phi_{1}(\sigma, \mathrm{u}(\sigma), \mathrm{z}(\sigma))\right| \leqslant \pi_{4}(\sigma)+\pi_{5}(\sigma)|\mathrm{u}(\sigma)|+\pi_{6}(\sigma)|\mathrm{z}(\sigma)|, \\
& =\pi_{4}(\sigma)+\pi_{5}(\sigma)\left|\mathrm{u}(\sigma)+\pi_{6}(\sigma)\right|\left|\phi_{2}(\sigma, \mathrm{v}(\sigma), \mathrm{y}(\sigma))\right| \\
& \leqslant \pi_{4}(\sigma)+\pi_{5}(\sigma)|\mathrm{u}(\sigma)|+\pi_{6}(\sigma) \pi_{7}(\sigma)+\pi_{6}(\sigma) \pi_{8}(\sigma)|\mathrm{v}(\sigma)|+\pi_{6}(\sigma) \pi_{9}(\sigma)|\mathrm{y}(\sigma)| .
\end{aligned}
$$

So, we obtain

Now, using 2 of Lemma 6, $\left(\mathbf{M}_{11}\right)$, and (91) in (89), we get

$|\mathrm{y}(\sigma)| \leqslant \frac{\pi_{4}(\sigma)+\pi_{6}(\sigma) \pi_{7}(\sigma)}{1-\pi_{6}(\sigma) \pi_{9}(\sigma)}+\frac{\pi_{5}(\sigma)|\mathrm{u}(\sigma)|+\pi_{6}(\sigma) \pi_{8}(\sigma)|\mathrm{v}(\sigma)|}{1-\pi_{6}(\sigma) \pi_{9}(\sigma)}$.

$$
\begin{aligned}
\left\|\mathfrak{I}_{\alpha}(\mathrm{u}, \mathrm{v})\right\|_{\mathbb{X} \times \mathbb{X}} \leqslant & {\left[\frac{\pi_{4}^{*}+\pi_{6}^{*} \pi_{7}^{*}}{1-\pi_{6}^{*} \pi_{9}^{*}}+\frac{\pi_{5}^{*}\|\mathrm{u}\|_{\mathbb{X}}+\pi_{6}^{*} \pi_{8}^{*}\|\mathrm{v}\|_{\mathbb{X}}}{1-\pi_{6}^{*} \pi_{9}^{*}}\right] } \\
& \times\left[\frac{(\ln 2)^{\alpha-1}}{\nabla_{\alpha} \Gamma(\alpha+1)}\left(\alpha \mathfrak{S}_{\alpha} \zeta(\ln 2) \sum_{k=0}^{\alpha-1-\beta}(-1)^{\alpha-1-\beta-k} \frac{(\alpha-1-\beta) !}{k !}(\ln \zeta)^{k}\right)\right. \\
& +\frac{\alpha \mathfrak{E}_{\alpha} \zeta}{\alpha-\beta} \sum_{k=0}^{\alpha-\beta}(-1)^{\alpha-\beta-k} \frac{(\alpha-\beta) !}{k !}(\ln \zeta)^{k} \\
& \left.\left.+\mathfrak{P}(\ln \rho)^{\alpha-1} \Gamma(\alpha-\beta)(\alpha(\ln 2)+(\ln \rho))+\nabla_{\alpha}(\alpha(\ln 2)+(\ln 2))\right)\right] \\
\leqslant & {\left[\frac{\pi_{4}^{*}+\pi_{6}^{*} \pi_{7}^{*}}{1-\pi_{6}^{*} \pi_{9}^{*}}+\frac{\pi_{5}^{*}\|\mathrm{u}\|_{\mathbb{X}}+\pi_{6}^{*} \pi_{8}^{*}\|\mathrm{v}\|_{\mathbb{X}}}{1-\pi_{6}^{*} \pi_{9}^{*}}\right] \mathscr{Y}_{\alpha} . }
\end{aligned}
$$

In the same way, we obtain

Thus, from (92) and (93), we get

$\left\|\mathfrak{I}_{\gamma}(\mathrm{u}, \mathrm{v})\right\|_{\mathbb{X} \times \mathbb{X}} \leqslant\left[\frac{\pi_{7}^{*}+\pi_{4}^{*} \pi_{9}^{*}}{1-\pi_{6}^{*} \pi_{9}^{*}}+\frac{\pi_{5}^{*} \pi_{9}^{*}\|\mathrm{u}\|_{\mathbb{X}}+\pi_{8}^{*}\|\mathrm{v}\|_{\mathbb{X}}}{1-\pi_{6}^{*} \pi_{9}^{*}}\right] \mathscr{Y}_{\gamma}$.

$$
\begin{aligned}
\|\mathfrak{T}(\mathrm{u}, \mathrm{v})\|_{\mathbb{X} \times \mathbb{X}} & \leqslant\left\|\mathfrak{T}_{\alpha}(\mathrm{u}, \mathrm{v})\right\|_{\mathbb{X} \times \mathbb{X}}+\left\|\mathfrak{T}_{\gamma}(\mathrm{u}, \mathrm{v})\right\|_{\mathbb{X} \times \mathbb{X}} \\
& \leqslant\left[\frac{\pi_{4}^{*}+\pi_{6}^{*} \pi_{7}^{*}}{1-\pi_{6}^{*} \pi_{9}^{*}}+\frac{\pi_{5}^{*}\|\mathrm{u}\|_{\mathbb{X}}+\pi_{6}^{*} \pi_{8}^{*}\|\mathrm{v}\|_{\mathbb{X}}}{1-\pi_{6}^{*} \pi_{9}^{*}}\right] \mathscr{Y}_{\alpha}+\left[\frac{\pi_{7}^{*}+\pi_{4}^{*} \pi_{9}^{*}}{1-\pi_{6}^{*} \pi_{9}^{*}}+\frac{\pi_{5}^{*} \pi_{9}^{*}\|\mathrm{u}\|_{\mathbb{X}}+\pi_{8}^{*}\|\mathrm{v}\|_{\mathbb{X}}}{1-\pi_{6}^{*} \pi_{9}^{*}}\right] \mathscr{Y}_{\gamma}=\mathscr{M} .
\end{aligned}
$$


Thus, $\mathfrak{I}$ is uniformly bounded. Now, we prove the operator $\mathfrak{T}$ is equicontinuous. For this, suppose $\sigma_{1}>\sigma_{2} \in J$ and $\mathrm{u}, \mathrm{v} \in \mathscr{\mathscr { B }}$; then,

$$
\begin{aligned}
& \left|\mathfrak{I}_{\alpha}(u, v)\left(\sigma_{1}\right)-\mathfrak{I}_{\alpha}(u, v)\left(\sigma_{2}\right)\right|, \\
& =\mid-\frac{\left(\ln \sigma_{1}\right)^{\alpha-1}}{\Delta_{\alpha} \Gamma(\alpha)} \int_{1}^{\zeta} \int_{1}^{2} \varphi_{\alpha}\left(\sigma_{1}\right)\left(\ln \sigma_{1}\right)^{\alpha-1-\beta} y(\eta) \frac{\mathrm{d} \eta}{\eta} \mathrm{d} \sigma_{1} \\
& +\frac{\left(\ln \sigma_{2}\right)^{\alpha-1}}{\Delta_{\alpha} \Gamma(\alpha)} \int_{1}^{\zeta} \int_{1}^{2} \varphi_{\alpha}\left(\sigma_{2}\right)\left(\ln \sigma_{2}\right)^{\alpha-1-\beta} y(\eta) \frac{\mathrm{d} \eta}{\eta} \mathrm{d} \sigma_{2} \\
& -\frac{\left(\ln \sigma_{2}\right)^{\alpha-1}}{\Delta_{\alpha} \Gamma(\alpha)} \int_{1}^{\zeta} \int_{1}^{\sigma_{2}} \varphi_{\alpha}\left(\sigma_{2}\right)\left(\ln \frac{\sigma_{2}}{\eta}\right)^{\alpha-1-\beta} y(\eta) \frac{\mathrm{d} \eta}{\eta} \mathrm{d} \sigma_{2} \\
& +\frac{\left(\ln \sigma_{1}\right)^{\alpha-1}}{\Delta_{\alpha} \Gamma(\alpha)} \int_{1}^{\zeta} \int_{1}^{\sigma_{1}} \varphi_{\alpha}\left(\sigma_{1}\right)\left(\ln \frac{\sigma_{1}}{\eta}\right)^{\alpha-1-\beta} y(\eta) \frac{\mathrm{d} \eta}{\eta} \mathrm{d} \sigma_{1} \\
& -\frac{\left(\ln \sigma_{1}\right)^{\alpha-1} \Gamma(\alpha-\beta)}{\Delta_{\alpha} \Gamma^{2}(\alpha)} \int_{1}^{2} \int_{1}^{2}\left(\ln \sigma_{1}\right)^{\alpha-1} y(\eta) \frac{\mathrm{d} \eta}{\eta} \mathrm{d} \mu\left(\sigma_{1}\right) \\
& +\frac{\left(\ln \sigma_{2}\right)^{\alpha-1} \Gamma(\alpha-\beta)}{\Delta_{\alpha} \Gamma^{2}(\alpha)} \int_{1}^{2} \int_{1}^{2}\left(\ln \sigma_{2}\right)^{\alpha-1} y(\eta) \frac{\mathrm{d} \eta}{\eta} \mathrm{d} \mu\left(\sigma_{2}\right) \\
& -\frac{\left(\ln \sigma_{2}\right)^{\alpha-1} \Gamma(\alpha-\beta)}{\Delta_{\alpha} \Gamma^{2}(\alpha)} \int_{1}^{2} \int_{1}^{\sigma_{2}}\left(\ln \frac{\sigma_{2}}{\eta}\right)^{\alpha-1} y(\eta) \frac{\mathrm{d} \eta}{\eta} \mathrm{d} \mu\left(\sigma_{2}\right) \\
& +\frac{\left(\ln \sigma_{1}\right)^{\alpha-1} \Gamma(\alpha-\beta)}{\Delta_{\alpha} \Gamma^{2}(\alpha)} \int_{1}^{2} \int_{1}^{\sigma_{1}}\left(\ln \frac{\sigma_{1}}{\eta}\right)^{\alpha-1} y(\eta) \frac{\mathrm{d} \eta}{\eta} \mathrm{d} \mu\left(\sigma_{1}\right) \\
& -\frac{\left(\ln \sigma_{1}\right)^{\alpha-1}-\left(\ln \sigma_{2}\right)^{\alpha-1}}{\Gamma(\alpha)} \int_{1}^{2} y(\eta) \frac{d \eta}{\eta}+\frac{1}{\Gamma(\alpha)} \int_{\sigma_{2}}^{\sigma_{1}}\left(\ln \frac{\sigma_{1}}{\eta}\right)^{\alpha-1} y(\eta) \frac{\mathrm{d} \eta}{\eta} \\
& -\frac{1}{\Gamma(\alpha)} \int_{1}^{\sigma_{2}}\left(\left(\ln \frac{\sigma_{2}}{\eta}\right)^{\alpha-1}-\left(\ln \frac{\sigma_{1}}{\eta}\right)^{\alpha-1}\right) z(\eta) \frac{\mathrm{d} \eta}{\eta} \\
& \leq\left[\frac{\pi_{4}^{*}+\pi_{6}^{*} \pi_{7}^{*}}{1-\pi_{6}^{*} \pi_{9}^{*}}+\frac{\pi_{5}^{*}\|u\|_{\mathbb{X}}+\pi_{6}^{*} \pi_{8}^{*}\|v\|_{\mathbb{X}}}{1-\pi_{6}^{*} \pi_{9}^{*}}\right]\left[\frac{\left(\left(\ln \sigma_{1}\right)^{\alpha-1}-\left(\ln \sigma_{2}\right)^{\alpha-1}\right)}{\nabla \Gamma(\alpha+1)}\right. \\
& \times\left(\alpha \mathfrak{S} \zeta(\ln 2) \sum_{k=0}^{\alpha-1-\beta}(-1)^{\alpha-1-\beta-k} \frac{(\alpha-1-\beta) !}{k !}(\ln \zeta)^{k}+\frac{\alpha \mathfrak{E} \zeta}{\alpha-\beta} \sum_{k=0}^{\alpha-\beta}(-1)^{\alpha-\beta-k} \frac{(\alpha-\beta) !}{k !}(\ln \zeta)^{k}\right. \\
& \left.\left.+\mathfrak{P}(\ln \rho)^{\alpha-1} \Gamma(\alpha-\beta)(\alpha(\ln 2)+(\ln \rho))+\nabla \alpha(\ln 2)\right)+\frac{\left(\left(\ln \sigma_{1}\right)^{\alpha}-\left(\ln \sigma_{2}\right)^{\alpha}\right)}{\Gamma(\alpha+1)}\right] .
\end{aligned}
$$


In the same way, we can show that

$$
\begin{aligned}
& \left|\mathfrak{I} \gamma(\mathrm{u}, \mathrm{v})\left(\sigma_{1}\right)-\mathfrak{I}_{\gamma}(\mathrm{u}, \mathrm{v})\left(\sigma_{2}\right)\right| \\
& \leqslant\left[\frac{\pi_{7}^{*}+\pi_{4}^{*} \pi_{9}^{*}}{1-\pi_{6}^{*} \pi_{9}^{*}}+\frac{\pi_{5}^{*} \pi_{9}^{*}\|\mathrm{u}\|_{\mathbb{X}}+\pi_{8}^{*}\|\mathrm{v}\|_{\mathbb{X}}}{1-\pi_{6}^{*} \pi_{9}^{*}}\right]\left[\frac{\left(\left(\ln \sigma_{1}\right)^{\gamma-1}-\left(\ln \sigma_{2}\right)^{\gamma-1}\right)}{\nabla \Gamma(\gamma+1)}\right. \\
& \times\left(\gamma \mathfrak{s} \xi(\ln 2) \sum_{k=0}^{\gamma-1-\delta}(-1)^{\gamma-1-\delta-k} \frac{(\gamma-1-\delta) !}{k !}(\ln \xi)^{k}+\frac{\gamma \mathfrak{s} \xi}{\gamma-\delta} \sum_{k=0}^{\gamma-\delta}(-1)^{\gamma-\delta-k} \frac{(\gamma-\delta) !}{k !}(\ln \xi)^{k}\right. \\
& \left.\left.+\mathfrak{P}(\ln \rho)^{\gamma-1} \Gamma(\gamma-\delta)(\gamma(\ln 2)+(\ln \rho))+\nabla \gamma(\ln 2)\right)+\frac{\left(\left(\ln \sigma_{1}\right)^{\gamma}-\left(\ln \sigma_{2}\right)^{\gamma}\right)}{\Gamma(\gamma+1)}\right] . \\
& \frac{\mathscr{Y}_{\alpha}\left(\mathfrak{Q}_{\phi_{1}}+\mathfrak{Q}_{\phi_{1}}^{*} \mathfrak{Q}_{\phi_{2}}\right)+\mathscr{Y}_{\gamma}\left(\mathfrak{Q}_{\phi_{1}} \mathfrak{R}_{\phi_{2}}^{*}+\mathfrak{Q}_{\phi_{2}}\right)}{1-\mathfrak{\Omega}_{\phi_{1}}^{*} \mathfrak{\mathfrak { Q }}_{\phi_{2}}^{*}}<1 .
\end{aligned}
$$

The right-hand sides of (94) and (96) approache to zero as $\sigma_{1} \longrightarrow \sigma_{2}$. Hence, by the Arzelà-Ascoli theorem, $\mathfrak{I}$ is equicontinuous and uniformly equicontinuous. Also, it is very easy to prove that $\mathfrak{I}(\mathscr{B}) \subset \mathscr{B}$. Therefore, $\mathfrak{I}$ is completely continuous.

Theorem 9. Under the hypothesis $\left(\mathbf{M}_{10}\right),\left(\mathbf{M}_{11}\right)$ and

The coupled system (7) has a unique solution.

Proof. Let $\mathrm{u}, \mathrm{v}, \widehat{\mathrm{u}}, \widehat{\mathrm{v}} \in \mathscr{C}$, and we consider

$$
\begin{aligned}
\left|\mathfrak{I}_{\alpha}(\mathrm{u}, \mathrm{v})(\sigma)-\mathfrak{I}_{\alpha}(\widehat{\mathrm{u}}, \widehat{\mathrm{v}})(\sigma)\right| \leqslant \mid & \frac{(\ln \sigma)^{\alpha-1}}{\Delta_{\alpha} \Gamma(\alpha)} \int_{1}^{\zeta} \int_{1}^{2} \varphi_{\alpha}(\sigma)(\ln \sigma)^{\alpha-1-\beta} \frac{\mathrm{d} \eta}{\eta} \mathrm{d} \sigma \\
& +\frac{(\ln \sigma)^{\alpha-1}}{\Delta_{\alpha} \Gamma(\alpha)} \int_{1}^{\zeta} \int_{1}^{\sigma} \varphi_{\alpha}(\sigma)\left(\ln \frac{\sigma}{\eta}\right)^{\alpha-1-\beta} \frac{\mathrm{d} \eta}{\eta} \mathrm{d} \sigma \\
& +\frac{(\ln \sigma)^{\alpha-1} \Gamma(\alpha-\beta)}{\Delta_{\alpha} \Gamma^{2}(\alpha)} \int_{1}^{2} \int_{1}^{2}(\ln \sigma)^{\alpha-1} \frac{\mathrm{d} \eta}{\eta} \mathrm{d} \mu(\sigma) \\
& +\frac{(\ln \sigma)^{\alpha-1} \Gamma(\alpha-\beta)}{\Delta_{\alpha} \Gamma^{2}(\alpha)} \int_{1}^{2} \int_{1}^{\sigma}\left(\ln \frac{\sigma}{\eta}\right)^{\alpha-1} \frac{\mathrm{d} \eta}{\eta} \mathrm{d} \mu(\sigma) \\
& +\frac{(\ln \sigma)^{\alpha-1}}{\Gamma(\alpha)} \int_{1}^{2} \frac{\mathrm{d} \eta}{\eta}+\frac{1}{\Gamma(\alpha)} \int_{1}^{\sigma}\left(\ln \frac{\sigma}{\eta}\right)^{\alpha-1} \frac{\mathrm{d} \eta}{\eta}|\operatorname{|y}(\eta)-\widehat{\mathrm{y}}(\eta)|,
\end{aligned}
$$

where

$$
\begin{aligned}
& \mathrm{y}(\sigma)=-\phi_{1}(\sigma, \mathrm{u}(\sigma), \mathbf{z}(\sigma)), \\
& \widehat{\mathrm{y}}(\sigma)=-\phi_{1}(\sigma, \widehat{\mathrm{u}}(\sigma), \widehat{\mathbf{z}}(\sigma)) .
\end{aligned}
$$

Now, using $\left(\mathbf{M}_{10}\right)$, 
Substituting (100) in (98) and taking $\sup _{\sigma \in J}$, we get

$$
\begin{aligned}
\left\|\mathfrak{T}_{\alpha}(\mathrm{u}, \mathrm{v})-\mathfrak{T}_{\alpha}(\widehat{\mathrm{u}}, \widehat{\mathrm{v}})\right\|_{\mathbb{X} \times \mathbb{X}} \leqslant & {\left[\frac{\mathcal{Y}_{\alpha}\left(\mathfrak{Q}_{\phi_{1}}+\mathfrak{R}_{\phi_{1}}^{*} \mathfrak{Q}_{\phi_{2}}\right)}{1-\mathfrak{R}_{\phi_{1}}^{*} \mathfrak{Q}_{\phi_{2}}^{*}}\right] } \\
& \cdot\|(\mathrm{u}, \mathrm{v})-(\widehat{\mathrm{u}}, \widehat{\mathrm{v}})\|_{\mathbb{X} \times \mathbb{X}} \cdot
\end{aligned}
$$

In the same way, we can obtain

$$
\begin{aligned}
\left\|\mathfrak{T}_{\gamma}(\mathrm{u}, \mathrm{v})-\mathfrak{T}_{\gamma}(\widehat{\mathrm{u}}, \widehat{\mathrm{v}})\right\|_{\mathbb{X} \times \mathbb{X}} \leqslant & {\left[\frac{\mathscr{Y}_{\gamma}\left(\mathfrak{Q}_{\phi_{1}}{\mathfrak{\mathfrak { Q } ^ { 2 }}}_{\phi_{2}}^{*}+\mathfrak{\Omega}_{\phi_{2}}\right)}{1-\mathfrak{2}_{\phi_{1}}^{*} \mathfrak{\mathfrak { Q }}_{\phi_{2}}^{*}}\right] } \\
& \cdot\|(\mathrm{u}, \mathrm{v})-(\widehat{\mathrm{u}}, \widehat{\mathrm{v}})\|_{\mathbb{X} \times \mathbb{X}} .
\end{aligned}
$$

So, from (101) and (102), we get

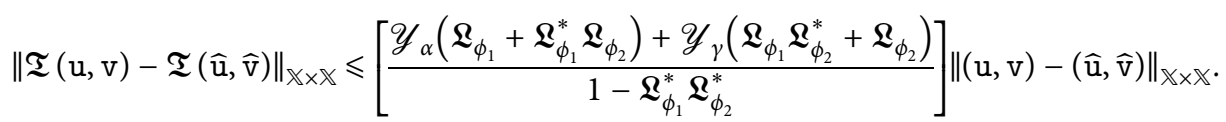

Thus, $\mathfrak{I}$ is a contraction. Therefore, by the Banach contraction principle, $\mathfrak{T}$ has a fixed point. So, we infer that the given coupled system (7) has a unique solution.
Theorem 10. In view of the continuity of the functions $\phi_{1}, \phi_{2}$ and supposing $\left(\mathbf{M}_{9}\right)$ and $\left(\mathbf{M}_{13}\right)$ with

$$
\begin{aligned}
& \mathbf{N}_{11}^{*}=\int_{1}^{2}\left|\mathbb{G}_{\alpha}(2, \eta)\right|\left(\frac{\pi_{4}^{*}+\pi_{6}^{*} \pi_{7}^{*}}{1-\pi_{6}^{*} \pi_{9}^{*}}\right) \frac{\mathrm{d} \eta}{\eta}<1, \quad \mathbf{N}_{12}^{*}=\int_{1}^{2}\left|\mathbb{G}_{\alpha}(2, \eta)\right|\left(\frac{\pi_{5}^{*}+\pi_{6}^{*} \pi_{8}^{*}}{1-\pi_{6}^{*} \pi_{9}^{*}}\right) \frac{\mathrm{d} \eta}{\eta}<1, \\
& \mathbf{N}_{21}^{*}=\int_{1}^{2}\left|\mathbb{G}_{\gamma}(2, \eta)\right|\left(\frac{\pi_{7}^{*}+\pi_{4}^{*} \pi_{9}^{*}}{1-\pi_{6}^{*} \pi_{9}^{*}}\right) \frac{\mathrm{d} \eta}{\eta}<1, \quad \mathbf{N}_{22}^{*}=\int_{1}^{2}\left|\mathbb{G}_{\gamma}(2, \eta)\right|\left(\frac{\pi_{5}^{*} \pi_{9}^{*}+\pi_{8}^{*}}{1-\pi_{6}^{*} \pi_{9}^{*}}\right) \frac{\mathrm{d} \eta}{\eta}<1
\end{aligned}
$$

hold, the coupled system (7) has at least one solution.

Proof. Let a set $\mathscr{B}$ be defined as

$$
\mathscr{\mathscr { B }}=\left\{(\mathrm{u}, \mathrm{v}) \in \mathbb{X} \times \mathbb{X}:\|(\mathrm{u}, \mathrm{v})\|_{\mathbb{X} \times \mathbb{X}}<\mathscr{R}_{\mathscr{B}}\right\},
$$

where $\max \left\{2 \mathbf{N}_{11}^{*} / 1-2 \mathbf{N}_{12}^{*}, 2 \mathbf{N}_{21}^{*} / 1-2 \mathbf{N}_{22}^{*}\right\}<\mathscr{R}_{\mathscr{B}}$. Furthermore, the operator defined by $\mathfrak{T}: \overline{\mathscr{B}} \longrightarrow \mathscr{\mathscr { C }}$ in (87) is completely continuous. Suppose $(\mathrm{u}, \mathrm{v}) \in \mathscr{\mathscr { B }}$; then, by definition of $\mathscr{\mathscr { B }}$, we have $\|(\mathrm{u}, \mathrm{v})\|_{\mathbb{X} \times \mathbb{X}}<\mathscr{R}_{\mathscr{B}}$;

$$
\begin{aligned}
\left\|\mathfrak{I}_{\alpha}(\mathrm{u}, \mathbf{z})\right\|_{\mathbb{X} \times \mathbb{X}} \leqslant & \max _{\sigma \in J} \int_{1}^{2}\left|\mathbb{G}_{\alpha}(\sigma, \eta)\right|\left|\phi_{1}(\eta, \mathrm{u}(\eta), \mathbf{z}(\eta))\right| \frac{\mathrm{d} \eta}{\eta} \\
\leqslant & \max _{\sigma \in J} \int_{1}^{2}\left|\mathbb{G}_{\alpha}(\sigma, \eta)\right|\left(\frac{\pi_{4}(\eta)+\pi_{6}(\eta) \pi_{7}(\eta)}{1-\pi_{6}(\eta) \pi_{9}(\eta)}\right) \frac{\mathrm{d} \eta}{\eta} \\
& +\max _{\sigma \in J} \int_{1}^{2}\left|\mathbb{G}_{\alpha}(\sigma, \eta)\right|\left(\frac{\pi_{5}(\eta)|\mathrm{u}(\eta)|+\pi_{6}(\eta) \pi_{8}(\eta)|\mathrm{v}(\eta)|}{1-\pi_{6}(\eta) \pi_{9}(\eta)}\right) \frac{\mathrm{d} \eta}{\eta} \\
\leqslant & \int_{1}^{2}\left|\mathbb{G}_{\alpha}(2, \eta)\right|\left(\frac{\pi_{4}^{*}+\pi_{6}^{*} \pi_{7}^{*}}{1-\pi_{6}^{*} \pi_{9}^{*}}\right) \frac{\mathrm{d} \eta}{\eta}+\mathscr{R}_{\mathscr{B}} \int_{1}^{2}\left|\mathbb{G}_{\alpha}(2, \eta)\right|\left(\frac{\pi_{5}^{*}+\pi_{6}^{*} \pi_{8}^{*}}{1-\pi_{6}^{*} \pi_{9}^{*}}\right) \frac{\mathrm{d} \eta}{\eta} \\
= & \mathbf{N}_{11}^{*}+\mathscr{R}_{\mathscr{B}} \mathbf{N}_{12}^{*} \leqslant \frac{\mathscr{R}_{\mathscr{B}}}{2}
\end{aligned}
$$

Similarly,

$$
\left\|\mathfrak{T}_{\gamma}(\mathrm{v}, \mathrm{y})\right\|_{\mathbb{X} \times \mathbb{X}} \leqslant \frac{\mathscr{R}_{\mathscr{\mathscr { B }}}}{2} .
$$

Therefore,

$$
\|\mathfrak{I}(\mathrm{u}, \mathrm{v})\|_{\mathbb{X} \times \mathbb{X}} \leqslant \mathscr{R}_{\mathscr{B}},
$$


so $\mathfrak{I}(\mathrm{u}, \mathrm{z}) \in \overline{\mathscr{B}}$. Thus, in view of Theorem 8, $\mathfrak{I}: \overline{\mathscr{B}} \longrightarrow \overline{\mathscr{B}}$ is completely continuous.

Now, we consider an eigenvalue problem defined as

$$
(\mathrm{u}, \mathrm{v})=\delta \mathfrak{I}(\mathrm{u}, \mathrm{v}), \quad \delta \in(0,1)
$$

$$
\begin{aligned}
\|\mathrm{u}\|_{\mathbb{X}}= & \left\|\delta \mathfrak{I}_{\alpha}(\mathrm{u}, \mathrm{z})\right\|_{\mathbb{X}} \leqslant \max _{\sigma \in J} \int_{1}^{2}\left|\mathbb{G}_{\alpha}(\sigma, \eta) \| \phi_{1}(\eta, \mathrm{u}(\eta), \mathbf{z}(\eta))\right| \frac{\mathrm{d} \eta}{\eta} \\
\leqslant & \max _{\sigma \in J} \int_{1}^{2}\left|\mathbb{G}_{\alpha}(\sigma, \eta)\right|\left(\frac{\pi_{4}(\eta)+\pi_{6}(\eta) \pi_{7}(\eta)}{1-\pi_{6}(\eta) \pi_{9}(\eta)}\right) \frac{\mathrm{d} \eta}{\eta} \\
& +\max _{\sigma \in J} \int_{1}^{2}\left|\mathbb{G}_{\alpha}(\sigma, \eta)\right|\left(\frac{\pi_{5}(\eta)|\mathrm{u}(\eta)|+\pi_{6}(\eta) \pi_{8}(\eta)|\mathrm{v}(\eta)|}{1-\pi_{6}(\eta) \pi_{9}(\eta)}\right) \frac{\mathrm{d} \eta}{\eta} \\
\leqslant & \int_{1}^{2}\left|\mathbb{G}_{\alpha}(2, \eta)\right|\left(\frac{\pi_{4}^{*}+\pi_{6}^{*} \pi_{7}^{*}}{1-\pi_{6}^{*} \pi_{9}^{*}}\right) \frac{\mathrm{d} \eta}{\eta}+\mathscr{R}_{\mathscr{B}} \int_{1}^{2}\left|\mathbb{G}_{\alpha}(2, \eta)\right|\left(\frac{\pi_{5}^{*}+\pi_{6}^{*} \pi_{8}^{*}}{1-\pi_{6}^{*} \pi_{9}^{*}}\right) \frac{\mathrm{d} \eta}{\eta} \\
= & \mathbf{N}_{11}^{*}+\mathscr{R}_{\mathscr{B}} \mathbf{N}_{12}^{*} \leqslant \frac{\mathscr{R}_{\mathscr{B}}}{2} .
\end{aligned}
$$

Similarly,

$$
|\mathrm{u}(\sigma)-\widehat{\mathrm{u}}(\sigma)| \leqslant \Psi(\varepsilon), \quad \sigma \in J .
$$

$$
\|\mathrm{v}\|_{\mathbb{X}} \leqslant \frac{\mathscr{R}_{\mathscr{B}}}{2}
$$

Thus,

$$
\|(\mathrm{u}, \mathrm{v})\|_{\mathbb{X} \times \mathbb{X}} \leqslant \mathscr{R}_{\mathscr{R}} .
$$

From equation (112), we get (u,v) $\notin \partial \mathscr{B}$. So, in view of Theorem $4, \mathfrak{I}$ has at least one fixed point which lies in $\overline{\mathscr{B}}$. This shows there is at least one solution of the coupled system (7).

\section{Hyers-Ulam Stability}

In this section, we provide novel characterizations of the Hyers-Ulam stability for systems (5) and (7). For the various concepts of Hyers-Ulam stability, see, for example, [37].

\subsection{Hyers-Ulam Stability Concepts for System (5)}

Definition 3. The problem (5) is said to be Hyers-Ulam stable if there exists some constant $\mathscr{K}>0$ such that, for any $\epsilon>0$ and for any solution $u \in \mathbb{X}$ of the inequality

$$
\left|\mathfrak{P}^{\alpha} \mathrm{u}(\sigma)+\phi\left(\sigma, \mathrm{u}(\sigma),{ }_{H} \mathfrak{D}^{\alpha} \mathrm{u}(\sigma)\right)\right| \leqslant \varepsilon, \quad \sigma \in J,
$$

there exists a solution $\widehat{\mathrm{u}} \in \mathbb{X}$ of (5) with

$$
|\mathrm{u}(\sigma)-\widehat{\mathrm{u}}(\sigma)| \leqslant \mathscr{K} \mathcal{\varepsilon}, \quad \sigma \in J .
$$

Definition 4. The problem (5) is said to be generalized Hyers-Ulam stable if there exists $\Psi \in C\left(\mathbb{R}^{+}, \mathbb{R}^{+}\right)$with $\Psi(0)=0$ such that, for any solution $\mathrm{u} \in \mathbb{X}$ of the inequality (113), there exists a solution $\widehat{u} \in \mathbb{X}$ of (5) satisfying

Definition 5. The problem (5) is said to be Hyers-Ulam-Rassias stable with respect to $\Psi \in C(J, \mathbb{R})$ if there exists some constant $\mathscr{K}_{\Psi}>0$ such that, for any $\epsilon>0$ and for any solution $u \in \mathbb{X}$ of the inequality

$$
\left|{ }_{H} \mathfrak{D}^{\alpha} \mathrm{u}(\sigma)+\phi\left(\sigma, \mathrm{u}(\sigma),{ }_{H} \mathfrak{D}^{\alpha} \mathrm{u}(\sigma)\right)\right| \leqslant \Psi(\sigma) \mathcal{\varepsilon}, \quad \sigma \in J,
$$

there exists a solution $\widehat{\mathrm{u}} \in \mathbb{X}$ of (5) with

$$
|\mathrm{u}(\sigma)-\widehat{\mathrm{u}}(\sigma)| \leqslant \mathscr{K}_{\Psi} \Psi(\sigma) \mathcal{E}, \quad \sigma \in J .
$$

Definition 6. The problem (5) is said to be generalized Hyers-Ulam-Rassias stable with respect to $\Psi \in C(J, \mathbb{R})$ if there exists some constant $\mathscr{K}_{\Psi}>0$ such that, for any solution $\mathrm{u} \in \mathbb{X}$ of the inequality (116), there exists a solution $\widehat{\mathrm{u}} \in \mathbb{X}$ of (5) satisfying

$$
|\mathrm{u}(\sigma)-\widehat{\mathrm{u}}(\sigma)| \leqslant \mathscr{K}_{\Psi} \Psi(\sigma), \quad \sigma \in J .
$$

Remark 1. Clearly,

(1) Definition $3 \Rightarrow$ Definition 4

(2) Definition $5 \Rightarrow$ Definition 6

Remark 2. Let $\mathrm{u} \in \mathbb{X}$ be a solution of the inequality (113); then, there exists a function $\mathscr{U}_{\phi} \in C(J, \mathbb{R})$ depending on $u$ such that
(1) $\left|\mathcal{U}_{\phi}(\sigma)\right| \leqslant \epsilon$, for all $\sigma \in J$
(2) ${ }_{H} \mathfrak{D}^{\alpha} \mathrm{u}(\sigma)+\phi\left(\sigma, \mathrm{u}(\sigma),{ }_{H} \mathfrak{D}^{\alpha} \mathrm{u}(\sigma)\right)=\mathscr{U}_{\phi}(\sigma), \sigma \in J$ 
Lemma 7. Let $2<\alpha \leqslant 3$; if $\mathrm{u} \in C^{1}(J, \mathbb{R})$ is the solution of the inequality (113), then $\mathrm{u}$ will be the solution of the following integral inequality:

$$
\left|\mathrm{u}(\sigma)-\omega_{1}(\sigma)\right| \leqslant \mathscr{Y} \epsilon
$$

Proof. Let $\mathrm{u}$ be the solution of the inequality (113). So, in view 2 of Remark 2, we have

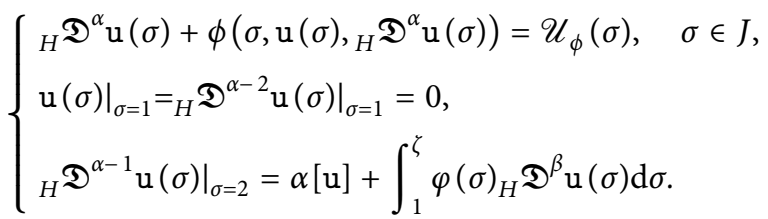

So, for $\sigma \in J$, the solution of (120) will be in the form

$$
\begin{aligned}
\mathrm{u}(\sigma)= & \int_{1}^{2} \mathbb{G}(\sigma, \eta) \phi\left(\eta, \mathrm{u}(\eta)_{{ }_{H}} \mathfrak{D}^{\alpha} \mathrm{u}(\eta)\right) \frac{\mathrm{d} \eta}{\eta} \\
& +\int_{1}^{2} \mathbb{G}(\sigma, \eta) \mathcal{U}_{\phi}(\eta) \frac{\mathrm{d} \eta}{\eta} .
\end{aligned}
$$

From equation (121), we have

$$
\begin{aligned}
\mathrm{u}(\sigma)= & -\frac{(\ln \sigma)^{\alpha-1}}{\Delta \Gamma(\alpha)}\left[\int_{1}^{\zeta} \int_{1}^{2} \varphi(\sigma)(\ln \sigma)^{\alpha-1-\beta} \mathrm{z}(\eta) \frac{\mathrm{d} \eta}{\eta} \mathrm{d} \sigma-\int_{1}^{\zeta} \int_{1}^{\sigma} \varphi(\sigma)\left(\ln \frac{\sigma}{\eta}\right)^{\alpha-1-\beta} \mathrm{z}(\eta) \frac{\mathrm{d} \eta}{\eta} \mathrm{d} \sigma\right] \\
& -\frac{(\ln \sigma)^{\alpha-1} \Gamma(\alpha-\beta)}{\Delta \Gamma^{2}(\alpha)}\left[\int_{1}^{2} \int_{1}^{2}(\ln \sigma)^{\alpha-1} \mathrm{z}(\eta) \frac{\mathrm{d} \eta}{\eta} \mathrm{d} \mu(\sigma)-\int_{1}^{2} \int_{1}^{\sigma}\left(\ln \frac{\sigma}{\eta}\right)^{\alpha-1} \mathrm{z}(\eta) \frac{\mathrm{d} \eta}{\eta} \mathrm{d} \mu(\sigma)\right] \\
& -\frac{(\ln \sigma)^{\alpha-1}}{\Gamma(\alpha)} \int_{1}^{2} \mathrm{z}(\eta) \frac{\mathrm{d} \eta}{\eta}+\frac{1}{\Gamma(\alpha)} \int_{1}^{\sigma}\left(\ln \frac{\sigma}{\eta}\right)^{\alpha-1} \mathrm{z}(\eta) \frac{\mathrm{d} \eta}{\eta} \\
& +\frac{(\ln \sigma)^{\alpha-1}}{\Delta \Gamma(\alpha)}\left[\int_{1}^{\zeta} \int_{1}^{2} \varphi(\sigma)(\ln \sigma)^{\alpha-1-\beta} \mathcal{U}_{\phi}(\eta) \frac{\mathrm{d} \eta}{\eta} \mathrm{d} \sigma-\int_{1}^{\zeta} \int_{1}^{\sigma} \varphi(\sigma)\left(\ln \frac{\sigma}{\eta}\right)^{\alpha-1-\beta} \mathcal{U}_{\phi}(\eta) \frac{\mathrm{d} \eta}{\eta} \mathrm{d} \sigma\right] \\
& +\frac{(\ln \sigma)^{\alpha-1} \Gamma(\alpha-\beta)}{\Delta \Gamma^{2}(\alpha)}\left[\int_{1}^{2} \int_{1}^{2}(\ln \sigma)^{\alpha-1} \mathcal{U}_{\phi}(\eta) \frac{\mathrm{d} \eta}{\eta} \mathrm{d} \mu(\sigma)-\int_{1}^{2} \int_{1}^{\sigma}\left(\ln \frac{\sigma}{\eta}\right)^{\alpha-1} \mathcal{U}_{\phi}(\eta) \frac{\mathrm{d} \eta}{\eta} \mathrm{d} \mu(\sigma)\right] \\
& +\frac{(\ln \sigma)^{\alpha-1}}{\Gamma(\alpha)} \int_{1}^{2} \mathcal{U}_{\phi}(\eta) \frac{\mathrm{d} \eta}{\eta}-\frac{1}{\Gamma(\alpha)} \int_{1}^{\sigma}\left(\ln \frac{\sigma}{\eta}\right)^{\alpha-1} \mathscr{U}_{\phi}(\eta) \frac{\mathrm{d} \eta}{\eta} \cdot
\end{aligned}
$$

For computational convenience, we use $\omega_{1}(\sigma)$ for the sum of terms which are free of $\mathcal{U}_{\phi}$, so we have

$$
\begin{aligned}
\omega_{1}(\sigma)= & -\frac{(\ln \sigma)^{\alpha-1}}{\Delta \Gamma(\alpha)}\left[\int_{1}^{\zeta} \int_{1}^{2} \varphi(\sigma)(\ln \sigma)^{\alpha-1-\beta} \mathbf{z}(\eta) \frac{\mathrm{d} \eta}{\eta} \mathrm{d} \sigma-\int_{1}^{\zeta} \int_{1}^{\sigma} \varphi(\sigma)\left(\ln \frac{\sigma}{\eta}\right)^{\alpha-1-\beta} \mathbf{z}(\eta) \frac{\mathrm{d} \eta}{\eta} \mathrm{d} \sigma\right] \\
& -\frac{(\ln \sigma)^{\alpha-1} \Gamma(\alpha-\beta)}{\Delta \Gamma^{2}(\alpha)}\left[\int_{1}^{2} \int_{1}^{2}(\ln \sigma)^{\alpha-1} \mathbf{z}(\eta) \frac{\mathrm{d} \eta}{\eta} \mathrm{d} \mu(\sigma)-\int_{1}^{2} \int_{1}^{\sigma}\left(\ln \frac{\sigma}{\eta}\right)^{\alpha-1} \mathbf{z}(\eta) \frac{\mathrm{d} \eta}{\eta} \mathrm{d} \mu(\sigma)\right] \\
& -\frac{(\ln \sigma)^{\alpha-1}}{\Gamma(\alpha)} \int_{1}^{2} \mathbf{z}(\eta) \frac{\mathrm{d} \eta}{\eta}+\frac{1}{\Gamma(\alpha)} \int_{1}^{\sigma}\left(\ln \frac{\sigma}{\eta}\right)^{\alpha-1} \mathbf{z}(\eta) \frac{\mathrm{d} \eta}{\eta} .
\end{aligned}
$$


From above, we have

$$
\begin{aligned}
& \left|\mathrm{u}(\sigma)-\omega_{1}(\sigma)\right| \leqslant \frac{(\ln \sigma)^{\alpha-1}}{|\Delta| \Gamma(\alpha)} \int_{1}^{\zeta} \int_{1}^{2}|\varphi(\sigma)|(\ln \sigma)^{\alpha-1-\beta} \mathscr{U}_{\phi}(\eta) \frac{\mathrm{d} \eta}{\eta} \mathrm{d} \sigma \\
& +\frac{(\ln \sigma)^{\alpha-1}}{|\Delta| \Gamma(\alpha)} \int_{1}^{\zeta} \int_{1}^{\sigma}|\varphi(\sigma)|\left(\ln \frac{\sigma}{\eta}\right)^{\alpha-1-\beta} \mid \mathscr{U}_{\phi}(\eta) \frac{\mathrm{d} \eta}{\eta} \mathrm{d} \sigma \\
& +\frac{(\ln \sigma)^{\alpha-1} \Gamma(\alpha-\beta)}{|\Delta| \Gamma^{2}(\alpha)} \int_{1}^{2} \int_{1}^{2}(\ln \sigma)^{\alpha-1} \mathscr{u}_{\phi}(\eta) \frac{\mathrm{d} \eta}{\eta} \mathrm{d} \mu(\sigma) \\
& +\frac{(\ln \sigma)^{\alpha-1} \Gamma(\alpha-\beta)}{|\Delta| \Gamma^{2}(\alpha)} \int_{1}^{2} \int_{1}^{\sigma}\left|\left(\ln \frac{\sigma}{\eta}\right)^{\alpha-1}\right| \mathcal{U}_{\phi}(\eta) \frac{\mathrm{d} \eta}{\eta} \mathrm{d} \mu(\sigma) \\
& +\frac{(\ln \sigma)^{\alpha-1}}{\Gamma(\alpha)} \int_{1}^{2} \mathscr{U}_{\phi}(\eta) \frac{d \eta}{\eta}+\frac{1}{\Gamma(\alpha)} \int_{1}^{\sigma}\left|\left(\ln \frac{\sigma}{\eta}\right)^{\alpha-1}\right| \mathscr{U}_{\phi}(\eta) \frac{d \eta}{\eta} \\
& \leqslant\left[\frac { ( \operatorname { l n } 2 ) ^ { \alpha - 1 } } { \nabla \Gamma ( \alpha + 1 ) } \left(\alpha \mathfrak{夭} \zeta(\ln 2) \sum_{k=0}^{\alpha-1-\beta}(-1)^{\alpha-1-\beta-k} \frac{(\alpha-1-\beta) !}{k !}(\ln \zeta)^{k}\right.\right. \\
& +\frac{\alpha \mathfrak{E} \zeta}{\alpha-\beta} \sum_{k=0}^{\alpha-\beta}(-1)^{\alpha-\beta-k} \frac{(\alpha-\beta) !}{k !}(\ln \zeta)^{k} \\
& \left.\left.+\mathfrak{P}(\ln \rho)^{\alpha-1} \Gamma(\alpha-\beta)(\alpha(\ln 2)+(\ln \rho))+\nabla(\alpha(\ln 2)+(\ln 2))\right)\right] \epsilon . \\
& \left|\mathrm{u}(\sigma)-\omega_{1}(\sigma)\right| \leqslant \mathscr{Y} \varepsilon . \\
& \left\{\begin{array}{l}
{ }_{H} \mathfrak{D}^{\alpha} \widehat{\mathrm{u}}(\sigma)+\phi\left(\sigma, \widehat{\mathrm{u}}(\sigma),{ }_{H} \mathfrak{D}^{\alpha} \widehat{\mathrm{u}}(\sigma)\right)=0, \quad \sigma \in J, \\
\left.\widehat{\mathrm{u}}(\sigma)\right|_{\sigma=1}=\left.{ }_{H} \mathfrak{D}^{\alpha-2} \widehat{\mathrm{u}}(\sigma)\right|_{\sigma=1}=0, \\
\left.{ }_{H} \mathfrak{D}^{\alpha-1} \widehat{\mathrm{u}}(\sigma)\right|_{\sigma=2}=\alpha[\widehat{\mathrm{u}}]+\int_{1}^{\zeta} \varphi(\sigma)_{H} \mathfrak{D}^{\beta} \widehat{\mathrm{u}}(\sigma) \mathrm{d} \sigma .
\end{array}\right.
\end{aligned}
$$

Using (3) of Lemma 4 and 1 of Remark 2, we get

Theorem 11. Under the hypothesis $\left(\mathbf{M}_{2}\right),\left(\mathbf{M}_{4}\right)$, and $\left(\mathbf{M}_{5}\right)$ and if

$$
\frac{\mathscr{y} \mathfrak{\Omega}_{\phi}}{1-\mathfrak{\Omega}_{\phi}^{*}}<1
$$

holds, then the given system (5) is $\mathscr{H} \mathscr{U} \mathcal{S}$ and, consequently, $\mathscr{G} \mathscr{H} \mathscr{U}$.

Proof. Let $\mathrm{u} \in C(J, \mathbb{R})$ be the solution of (113) and $\mathrm{u}$ be the unique solution of the system given by

Then, for $\sigma \in J$, the solution of (127) is

$$
\widehat{\mathrm{u}}(\sigma)=\int_{1}^{2} \mathbb{G}(\sigma, \eta) \phi\left(\eta, \widehat{\mathrm{u}}(\eta)_{H} \mathfrak{D}^{\alpha} \widehat{\mathrm{u}}(\eta)\right) \frac{\mathrm{d} \eta}{\eta} .
$$

\section{Consider}

$|\mathrm{u}(\sigma)-\widehat{\mathrm{u}}(\sigma)| \leqslant\left|\mathrm{u}(\sigma)-\omega_{1}(\sigma)\right|+\left|\omega_{1}(\sigma)-\widehat{\mathrm{u}}(\sigma)\right|$.

Using Lemma 7 in (129), we have

$$
\begin{aligned}
& |\mathrm{u}(\sigma)-\widehat{\mathrm{u}}(\sigma)| \\
& \leqslant \mathscr{Y} \epsilon+\mid \frac{(\ln \sigma)^{\alpha-1}}{\Delta \Gamma(\alpha)} \int_{1}^{\zeta} \int_{1}^{2} \varphi(\sigma)(\ln \sigma)^{\alpha-1-\beta} \frac{\mathrm{d} \eta}{\eta} \mathrm{d} \sigma+\frac{(\ln \sigma)^{\alpha-1}}{\Delta \Gamma(\alpha)} \int_{1}^{\zeta} \int_{1}^{\sigma} \varphi(\sigma)\left(\ln \frac{\sigma}{\eta}\right)^{\alpha-1-\beta} \frac{\mathrm{d} \eta}{\eta} \mathrm{d} \sigma \\
& \quad+\frac{(\ln \sigma)^{\alpha-1} \Gamma(\alpha-\beta)}{\Delta \Gamma^{2}(\alpha)} \int_{1}^{2} \int_{1}^{2}(\ln \sigma)^{\alpha-1} \frac{\mathrm{d} \eta}{\eta} \mathrm{d} \mu(\sigma)+\frac{(\ln \sigma)^{\alpha-1} \Gamma(\alpha-\beta)}{\Delta \Gamma^{2}(\alpha)} \int_{1}^{2} \int_{1}^{\sigma}\left(\ln \frac{\sigma}{\eta}\right)^{\alpha-1} \frac{\mathrm{d} \eta}{\eta} \mathrm{d} \mu(\sigma) \\
& \quad+\frac{(\ln \sigma)^{\alpha-1}}{\Gamma(\alpha)} \int_{1}^{2} \frac{\mathrm{d} \eta}{\eta}+\frac{1}{\Gamma(\alpha)} \int_{1}^{\sigma}\left(\ln \frac{\sigma}{\eta}\right)^{\alpha-1} \frac{\mathrm{d} \eta}{\eta}|| \mathrm{z}_{\mathrm{u}}(\eta)-\mathrm{z}_{\widehat{\mathrm{u}}}(\eta) \mid,
\end{aligned}
$$


where $z_{\mathrm{u}}, \mathrm{z}_{\widehat{\mathrm{u}}} \in C(J, \mathbb{R})$ are of the form

$$
\begin{aligned}
& \mathbf{z}_{\mathrm{u}}(\sigma)=-\phi\left(\sigma, \mathrm{u}(\sigma), \mathbf{z}_{\mathrm{u}}(\sigma)\right), \\
& \mathbf{z}_{\widehat{\mathrm{u}}}(\sigma)=-\phi\left(\sigma, \widehat{\mathrm{u}}(\sigma), \mathbf{z}_{\widehat{\mathrm{u}}}(\sigma)\right) .
\end{aligned}
$$

By $\left(\mathbf{M}_{4}\right)$, we get

$$
\begin{aligned}
\left|\mathbf{z}_{\mathrm{u}}(\sigma)-\mathbf{z}_{\widehat{\mathrm{u}}}(\sigma)\right| & =\left|-\left(\phi\left(\sigma, \mathrm{u}(\sigma), \mathbf{z}_{\mathrm{u}}(\sigma)\right)-\phi\left(\sigma, \widehat{\mathrm{u}}(\sigma), \mathbf{z}_{\widehat{\mathrm{u}}}(\sigma)\right)\right)\right| \\
& \leqslant \mathfrak{Q}_{\phi}|\mathrm{u}(\sigma)-\widehat{\mathrm{u}}(\sigma)|+{\mathfrak{\mathfrak { Q } _ { \phi }}}_{\phi}^{*}\left|\mathbf{z}_{\mathrm{u}}(\sigma)-\mathbf{z}_{\widehat{\mathrm{u}}}(\sigma)\right|,
\end{aligned}
$$

which implies

$$
\left|z_{\mathrm{u}}(\sigma)-\mathrm{z}_{\widehat{\mathrm{u}}}(\sigma)\right| \leqslant \frac{\mathfrak{Q}_{\phi}}{1-\mathfrak{\Omega}_{\phi}^{*}}|\mathrm{u}(\sigma)-\widehat{\mathrm{u}}(\sigma)| .
$$

Using (3) of Lemma 4 and (133) in (130), we get

$$
\begin{aligned}
& \|\mathrm{u}-\widehat{\mathrm{u}}\|_{\mathbb{X}} \leqslant \mathscr{Y} \epsilon+\frac{\mathscr{Y} \mathfrak{\Omega}_{\phi}}{1-\mathfrak{\Omega}_{\phi}^{*}}\|\mathrm{u}-\widehat{\mathrm{u}}\|_{\mathbb{X}}, \\
& \|\mathrm{u}-\widehat{\mathrm{u}}\|_{\mathbb{X}} \leqslant \frac{\mathscr{Y}\left(1-\mathfrak{\Omega}_{\phi}^{*}\right)}{1-\mathfrak{\Omega}_{\phi}^{*}-\mathscr{Y} \mathfrak{\Omega}_{\phi}} \epsilon=\mathscr{K} \epsilon,
\end{aligned}
$$

where

$$
\mathscr{K}=\frac{\mathscr{Y}\left(1-\mathfrak{\Omega}_{\phi}^{*}\right)}{1-\mathfrak{\Omega}_{\phi}^{*}-\mathscr{Y}_{\phi}},
$$

such that

$$
\frac{\mathscr{Y} \mathfrak{\Omega}_{\phi}}{1-\mathfrak{\Omega}_{\phi}^{*}}<1 .
$$

Thus, problem (5) is $\mathscr{H} \mathscr{U} \mathcal{S}$.

Remark 3. By setting $\Psi(\epsilon)=\mathscr{K} \epsilon, \Psi(0)=0$ in (134), by Definition 4, the given system (5) is $\mathscr{G} \mathscr{H} \mathscr{U} \mathcal{S}$.

Lemma 8. Let the hypothesis $\left(\mathbf{M}_{6}\right)$ hold, and suppose $\mathrm{u} \in C(J, \mathbb{R})$ is the solution of the inequality (116); then, $\mathrm{u}$ is a solution of the following inequality:

$$
\left|\mathrm{u}(\sigma)-\omega_{1}(\sigma)\right| \leqslant \mathscr{y} \Omega_{\Psi} \Psi(\sigma) \epsilon .
$$

Proof. From Lemma 7, we have

$$
\begin{aligned}
\left|\mathrm{u}(\sigma)-\omega_{1}(\sigma)\right| \leqslant & \frac{(\ln \sigma)^{\alpha-1}}{|\Delta| \Gamma(\alpha)} \int_{1}^{\zeta} \int_{1}^{2}|\varphi(\sigma)|(\ln \sigma)^{\alpha-1-\beta} \mathscr{U}_{\phi}(\eta) \frac{\mathrm{d} \eta}{\eta} \mathrm{d} \sigma \\
& +\frac{(\ln \sigma)^{\alpha-1}}{|\Delta| \Gamma(\alpha)} \int_{1}^{\zeta} \int_{1}^{\sigma}|\varphi(\sigma)|\left(\ln \frac{\sigma}{\eta}\right)^{\alpha-1-\beta} \mid \mathcal{U}_{\phi}(\eta) \frac{\mathrm{d} \eta}{\eta} \mathrm{d} \sigma \\
& +\frac{(\ln \sigma)^{\alpha-1} \Gamma(\alpha-\beta)}{|\Delta| \Gamma^{2}(\alpha)} \int_{1}^{2} \int_{1}^{2}(\ln \sigma)^{\alpha-1} \mathscr{U}_{\phi}(\eta) \frac{\mathrm{d} \eta}{\eta} \mathrm{d} \mu(\sigma) \\
& +\frac{(\ln \sigma)^{\alpha-1} \Gamma(\alpha-\beta)}{|\Delta| \Gamma^{2}(\alpha)} \int_{1}^{2} \int_{1}^{\sigma}\left|\left(\ln \frac{\sigma}{\eta}\right)^{\alpha-1}\right| \mathcal{U}_{\phi}(\eta) \frac{\mathrm{d} \eta}{\eta} \mathrm{d} \mu(\sigma) \\
& +\frac{(\ln \sigma)^{\alpha-1}}{\Gamma(\alpha)} \int_{1}^{2} \mathscr{U}_{\phi}(\eta) \frac{\mathrm{d} \eta}{\eta}+\frac{1}{\Gamma(\alpha)} \int_{1}^{\sigma}\left|\left(\ln \frac{\sigma}{\eta}\right)^{\alpha-1}\right| \mathscr{U}_{\phi}(\eta) \frac{\mathrm{d} \eta}{\eta} .
\end{aligned}
$$

By using (3) of Lemma 4, 1 of Remark 2, and $\left(\mathbf{M}_{6}\right)$, we get

$$
\begin{aligned}
\left|\mathrm{u}(\sigma)-\omega_{1}(\sigma)\right| \leqslant & {\left[\frac { ( \operatorname { l n } 2 ) ^ { \alpha - 1 } } { \nabla \Gamma ( \alpha + 1 ) } \left(\alpha \mathfrak{\xi} \zeta(\ln 2) \sum_{k=0}^{\alpha-1-\beta}(-1)^{\alpha-1-\beta-k} \frac{(\alpha-1-\beta) !}{k !}(\ln \zeta)^{k}\right.\right.} \\
& +\frac{\alpha \mathfrak{E} \zeta}{\alpha-\beta} \sum_{k=0}^{\alpha-\beta}(-1)^{\alpha-\beta-k} \frac{(\alpha-\beta) !}{k !}(\ln \zeta)^{k} \\
& \left.\left.+\mathfrak{P}(\ln \rho)^{\alpha-1} \Gamma(\alpha-\beta)(\alpha(\ln 2)+(\ln \rho))+\nabla(\alpha(\ln 2)+(\ln 2))\right)\right] \Omega_{\Psi} \Psi(\sigma) \epsilon \\
\leqslant & \mathscr{Y} \Omega_{\Psi} \Psi(\sigma) \epsilon .
\end{aligned}
$$


Theorem 12. Under the hypothesis $\left(\mathbf{M}_{2}\right)$ and $\left(\mathbf{M}_{4}\right)-\left(\mathbf{M}_{6}\right)$ and if the inequality

$$
\frac{1-\mathbf{\Omega}_{\phi}^{*}-\mathscr{Y} \mathfrak{Q}_{\phi}}{1-\mathfrak{\Omega}_{\phi}^{*}}>0
$$

holds, then the given system (5) is stable in the sense of $\mathscr{H} \mathscr{U}$.

Proof. Let $\mathrm{u} \in C(J, \mathbb{R})$ be the solution of (113) and $\widehat{\mathrm{u}}$ be the unique solution of the system given by

$$
\left\{\begin{array}{l}
{ }_{H} \mathfrak{D}^{\alpha} \widehat{\mathrm{u}}(\sigma)+\phi\left(\sigma, \widehat{\mathrm{u}}(\sigma),{ }_{H} \mathfrak{D}^{\alpha} \widehat{\mathrm{u}}(\sigma)\right)=0, \quad \sigma \in J, \\
\left.\widehat{\mathrm{u}}(\sigma)\right|_{\sigma=1}=\left.{ }_{H} \mathfrak{D}^{\alpha-2} \widehat{\mathrm{u}}(\sigma)\right|_{\sigma=1}=0, \\
\left.{ }_{H} \mathfrak{D}^{\alpha-1} \widehat{\mathrm{u}}(\sigma)\right|_{\sigma=2}=\alpha[\widehat{\mathrm{u}}]+\int_{1}^{\zeta} \varphi(\sigma)_{H} \mathfrak{D}^{\beta} \widehat{\mathrm{u}}(\sigma) \mathrm{d} \sigma .
\end{array}\right.
$$

Then, for $\sigma \in J$, the solution of (141) is

$$
\widehat{\mathrm{u}}(\sigma)=\int_{1}^{2} \mathbb{G}(\sigma, \eta) \phi\left(\eta, \widehat{\mathrm{u}}(\eta)_{H_{H}} \mathfrak{D}^{\alpha} \widehat{\mathrm{u}}(\eta)\right) \frac{\mathrm{d} \eta}{\eta} .
$$

\section{Consider}

$|\mathrm{u}(\sigma)-\widehat{\mathrm{u}}(\sigma)| \leqslant\left|\mathrm{u}(\sigma)-\omega_{1}(\sigma)\right|+\left|\omega_{1}(\sigma)-\widehat{\mathrm{u}}(\sigma)\right|$.

Using $\left(\mathbf{M}_{4}\right)$ in a similar way as used in Theorem 11 , we get

$$
\left|\omega_{1}(\sigma)-\widehat{\mathrm{u}}(\sigma)\right| \leqslant \frac{\mathcal{Y} \mathfrak{\Omega}_{\phi}}{1-\mathfrak{\Omega}_{\phi}^{*}}|\mathrm{u}(\eta)-\widehat{\mathrm{u}}(\eta)| .
$$

Now, by Lemma 8 and by (144), (143) becomes

$$
\|\mathrm{u}-\widehat{\mathrm{u}}\|_{\mathbb{X}} \leqslant \mathscr{Y} \Omega_{\Psi} \Psi(\sigma) \epsilon+\frac{\mathcal{Y} \mathfrak{\Omega}_{\phi}}{1-\mathfrak{\Omega}_{\phi}^{*}}\|\mathrm{u}-\widehat{\mathrm{u}}\|_{\mathbb{X}}
$$

which implies

$$
\|\mathrm{u}-\widehat{\mathrm{u}}\|_{\mathbb{X}} \leqslant \frac{1-\mathfrak{\Omega}_{\phi}^{*}}{1-\mathfrak{\Omega}_{\phi}^{*}-\mathcal{Y} \mathfrak{\Omega}_{\phi}} \mathscr{Y} \Omega_{\Psi} \Psi(\sigma) \epsilon .
$$

Thus, we have

$$
\|\mathrm{u}-\widehat{\mathrm{u}}\|_{\mathcal{X}} \leqslant \mathscr{K}_{\Psi} \Psi(\sigma) \epsilon,
$$

where

$$
\begin{aligned}
\mathscr{K}_{\Psi} & =\frac{1-\mathfrak{\Omega}_{\phi}^{*}}{1-\mathfrak{\Omega}_{\phi}^{*}-\mathcal{Y} \mathbf{\Omega}_{\phi}} \mathcal{Y} \Omega_{\Psi}, \\
\frac{1-\mathfrak{\Omega}_{\phi}^{*}-\mathcal{Y} \mathbf{\Omega}_{\phi}}{1-\mathfrak{\Omega}_{\phi}^{*}}>0 . &
\end{aligned}
$$

Hence, the given system (5) is $\mathscr{H} \mathscr{U} \mathscr{R}$ stable.

Remark 4. If $\epsilon=1$ in (147), then by Definition 6, the given system (5) is $\mathscr{G} \mathscr{H} \mathscr{U} \mathscr{S}$.

\subsection{Hyers-Ulam Stability Concepts for System (7)}

Definition 7 (see [38]). The given system (7) has $\mathscr{H} \mathscr{U} \mathcal{S}$ if there exists $\mathfrak{E}_{\alpha, \gamma}=\max \left(\mathfrak{E}_{\alpha}, \mathfrak{c}_{\gamma}\right)>0$ such that there exist some $\varepsilon_{\alpha, \gamma}=\max \left(\varepsilon_{\alpha}, \varepsilon_{\gamma}\right)>0$, and for every solution $(\mathrm{u}, \mathrm{v}) \in \mathbb{X} \times \mathbb{X}$ of the inequality

$$
\begin{cases}\left|\mathfrak{D}^{\alpha} \mathrm{u}(\sigma)+\phi_{1}\left(\sigma, \mathrm{u}(\sigma),{ }_{H} \mathfrak{D}^{\gamma} \mathrm{v}(\sigma)\right)\right| \leqslant \epsilon_{\alpha}, & \sigma \in J, \\ \left|\mathfrak{D}^{\gamma} \mathrm{v}(\sigma)+\phi_{2}\left(\sigma, \mathrm{v}(\sigma),{ }_{H} \mathfrak{D}^{\alpha} \mathrm{u}(\sigma)\right)\right| \leqslant \epsilon_{\gamma}, & \sigma \in J,\end{cases}
$$

there exists a solution $(\widehat{\mathrm{u}}, \widehat{\mathrm{v}}) \in \mathbb{X} \times \mathbb{X}$ with

$$
\|(\mathrm{u}, \mathrm{v})(\sigma)-(\widehat{\mathrm{u}}, \widehat{\mathrm{v}})(\sigma)\| \leqslant \mathfrak{夭}_{\alpha, \gamma} \epsilon_{\alpha, \gamma}, \quad \sigma \in J .
$$

Definition 8 (see [38]). The given system (7) has $\mathscr{G} \mathscr{H} \mathscr{U} \mathcal{S}$ if there exists $\mathfrak{V}^{\prime} \in C\left(\mathbb{R}^{+}, \mathbb{R}^{+}\right)$with $\mathfrak{C}^{\prime}(0)=0$ such that, for any solution $(\mathrm{u}, \mathrm{v}) \in \mathbb{X} \times \mathbb{X}$ of the inequality (149), there exists a solution ( $\widehat{\mathrm{u}}, \widehat{\mathrm{v}}) \in \mathbb{X} \times \mathbb{X}$ of (7) satisfying

$$
\|(\mathrm{u}, \mathrm{v})(\sigma)-(\widehat{\mathrm{u}}, \widehat{\mathrm{v}})(\sigma)\| \leqslant \mathfrak{V}^{\prime}\left(\epsilon_{\alpha, \gamma}\right), \quad \sigma \in J .
$$

Definition 9 (see [38]). The given system (7) has $\mathscr{H} \mathscr{U} \mathscr{R} \mathcal{S}$ with respect to $\Psi_{\alpha, \gamma}=\max \left(\Psi_{\alpha}, \Psi_{\gamma}\right)$ with $\Psi_{\alpha, \gamma} \in C(J, \mathbb{R})$ if there exists some constant $\mathfrak{G}_{\Psi_{\alpha \gamma}}=\max \left(\mathfrak{G}_{\Psi_{\alpha}}, \mathfrak{S}_{\Psi_{\gamma}}\right)>0$ such that, for any $\varepsilon_{\alpha, \gamma}=\max \left(\varepsilon_{\alpha}, \varepsilon_{\gamma}^{\alpha, \gamma}\right)^{\prime \gamma}>0$ and for any solution $(\mathrm{u}, \mathrm{v}) \in \mathbb{X} \times \mathbb{X}$ of the inequalities

$$
\begin{cases}\left|\mathfrak{D}^{\alpha} \mathrm{u}(\sigma)+\phi_{1}\left(\sigma, \mathrm{u}(\sigma),{ }_{H} \mathfrak{D}^{\gamma} \mathrm{v}(\sigma)\right)\right| \leqslant \Psi_{\alpha}(\sigma) \epsilon_{\alpha}, & \sigma \in J, \\ \left|\mathfrak{D}^{\gamma} \mathrm{v}(\sigma)+\phi_{2}\left(\sigma, \mathrm{v}(\sigma),{ }_{H} \mathfrak{D}^{\alpha} \mathrm{u}(\sigma)\right)\right| \leqslant \Psi_{\gamma}(\sigma) \epsilon_{\gamma}, & \sigma \in J,\end{cases}
$$

there exists a solution $(\widehat{\mathrm{u}}, \widehat{\mathrm{v}}) \in \mathbb{X} \times \mathbb{X}$ with

$$
\|(\mathrm{u}, \mathrm{v})(\sigma)-(\widehat{\mathrm{u}}, \widehat{\mathrm{v}})(\sigma)\| \leqslant \mathfrak{夭}_{\Psi_{\alpha, \gamma}} \Psi_{\alpha, \gamma}(\sigma) \epsilon_{\alpha, \gamma}, \quad \sigma \in J .
$$

Definition 10 (see [38]). The given system (7) has $\mathscr{G} \mathscr{H} \mathscr{U} \mathscr{S}$ with respect to $\Psi_{\alpha, \gamma}=\max \left(\Psi_{\alpha}, \Psi_{\gamma}\right)$ with $\Psi_{\alpha, \gamma} \in C(J, \mathbb{R})$ if there exists some constant $\mathfrak{G}_{\Psi_{\alpha}}=\max \left(\mathfrak{C}_{\Psi_{\alpha}}, \mathfrak{V}_{\Psi_{\gamma}}\right)>0$ such that, for any solution $(\widehat{\mathrm{u}}, \widehat{\mathrm{v}}) \in \mathbb{X}^{\alpha, \gamma} \times \mathbb{X}$ of the inequality (152), there exists a solution $(\mathrm{u}, \mathrm{v}) \in \mathbb{X} \times \mathbb{X}$ of (7) satisfying

$$
\|(\mathrm{u}, \mathrm{v})(\sigma)-(\widehat{\mathrm{u}}, \widehat{\mathrm{v}})(\sigma)\| \leqslant \boldsymbol{C}_{\Psi_{\alpha, \gamma}} \Psi_{\alpha, \gamma}(\sigma), \quad \sigma \in J
$$

Remark 5. Let $(\mathrm{u}, \mathrm{v}) \in \mathbb{X} \times \mathbb{X}$ be a solution of the inequality (149), if there exist functions $\mathcal{U}_{\phi_{1}}, \mathcal{U}_{\phi_{2}} \in C(J, \mathbb{R})$ depending on $\mathrm{u}, \mathrm{v}$, respectively, such that

$$
\begin{aligned}
& \text { (1) }\left|\mathscr{U}_{\phi_{1}}(\sigma)\right| \leqslant \epsilon_{\alpha},\left|\mathcal{U}_{\phi_{2}}(\sigma)\right| \leqslant \epsilon_{\gamma}, \sigma \in J \\
& \text { (2) }\left\{\begin{array}{l}
{ }_{H} \mathfrak{D}^{\alpha} \mathrm{u}(\sigma)+\phi_{1}\left(\sigma, \mathrm{u}(\sigma),{ }_{H} \mathfrak{D}^{\gamma} \mathrm{v}(\sigma)\right)=\mathscr{U}_{\phi_{1}}(\sigma), \sigma \in J \\
\mathfrak{H}^{\gamma} \mathrm{v}(\sigma)+\phi_{2}\left(\sigma, \mathrm{v}(\sigma),{ }_{H} \mathfrak{D}^{\alpha} \mathrm{u}(\sigma)\right)=\mathscr{U}_{\phi_{2}}(\sigma), \sigma \in J
\end{array}\right.
\end{aligned}
$$

Lemma 9. Let $(\mathrm{u}, \mathrm{v}) \in \mathbb{X} \times \mathbb{X}$ be the solution of (149); then, for $\sigma \in J$, we have 


$$
\left\{\begin{array}{l}
\left|\mathrm{u}(\sigma)-\omega_{1}(\sigma)\right| \leqslant \mathscr{Y}_{\alpha} \epsilon_{\alpha}, \\
\left|\mathrm{v}(\sigma)-\omega_{2}(\sigma)\right| \leqslant \mathscr{Y}_{\gamma} \epsilon_{\gamma} .
\end{array}\right.
$$

Proof. By 2 of Remark 5 and for $\sigma \in J$, we have

$$
\left\{\begin{array}{l}
{ }_{H} \mathfrak{D}^{\alpha} \mathrm{u}(\sigma)+\phi_{1}\left(\sigma, \mathrm{u}(\sigma),{ }_{H} \mathfrak{D}^{\gamma} \mathrm{v}(\sigma)\right)=\mathscr{U}_{\phi_{1}}(\sigma), \quad \sigma \in J, \\
{ }_{H} \mathfrak{D}^{\gamma} \mathrm{v}(\sigma)+\phi_{2}\left(\sigma, \mathrm{v}(\sigma),{ }_{H} \mathfrak{D}^{\alpha} \mathrm{u}(\sigma)\right)=\mathscr{U}_{\phi_{2}}(\sigma), \quad \sigma \in J, \\
\left.\mathrm{u}(\sigma)\right|_{\sigma=1}=\left.{ }_{H} \mathfrak{D}^{\alpha-2} \mathrm{u}(\sigma)\right|_{\sigma=1}=0,\left.{ }_{H} \mathfrak{D}^{\alpha-1} \mathrm{u}(\sigma)\right|_{\sigma=2}=\alpha[\mathrm{u}]+\int_{1}^{\zeta} \varphi_{\alpha}(\sigma)_{H} \mathfrak{D}^{\beta} \mathrm{u}(\sigma) \mathrm{d} \sigma, \\
\left.\mathrm{v}(\sigma)\right|_{\sigma=1}=\left.{ }_{H} \mathfrak{D}^{\gamma-2} \mathrm{v}(\sigma)\right|_{\sigma=1}=0,\left.{ }_{H} \mathfrak{D}^{\gamma-1} \mathrm{v}(\sigma)\right|_{\sigma=2}=\gamma[\mathrm{v}]+\int_{1}^{\xi} \varphi_{\gamma}(\sigma)_{H} \mathfrak{D}^{\beta} \mathrm{v}(\sigma) \mathrm{d} \sigma .
\end{array}\right.
$$

So, for $\sigma \in J$, the solution of (156) will be in the form

$$
\left\{\begin{array}{l}
\left.\mathrm{u}(\sigma)=\int_{1}^{2} \mathbb{G}_{\alpha}(\sigma, \eta) \phi_{1}(\eta, \mathrm{u}(\eta))_{H} \mathfrak{D}^{\gamma} \mathrm{v}(\eta)\right) \frac{\mathrm{d} \eta}{\eta}+\int_{1}^{2} \mathbb{G}_{\alpha}(\sigma, \eta) \mathcal{U}_{\phi_{1}}(\eta) \frac{\mathrm{d} \eta}{\eta} \\
\left.\mathrm{v}(\sigma)=\int_{1}^{2} \mathbb{G}_{\gamma}(\sigma, \eta) \phi_{2}(\eta, \mathrm{v}(\eta))_{H} \mathfrak{D}^{\alpha} \mathrm{u}(\eta)\right) \frac{\mathrm{d} \eta}{\eta}+\int_{1}^{2} \mathbb{G}_{\gamma}(\sigma, \eta) \mathcal{U}_{\phi_{2}}(\eta) \frac{\mathrm{d} \eta}{\eta}
\end{array}\right.
$$

From the first equation of system (157), we have

$$
\begin{aligned}
\mathrm{u}(\sigma)= & -\frac{(\ln \sigma)^{\alpha-1}}{\Delta_{\alpha} \Gamma(\alpha)}\left[\int_{1}^{\zeta} \int_{1}^{2} \varphi_{\alpha}(\sigma)(\ln \sigma)^{\alpha-1-\beta} \mathrm{y}(\eta) \frac{\mathrm{d} \eta}{\eta} \mathrm{d} \sigma-\int_{1}^{\zeta} \int_{1}^{\sigma} \varphi_{\alpha}(\sigma)\left(\ln \frac{\sigma}{\eta}\right)^{\alpha-1-\beta} \mathrm{y}(\eta) \frac{\mathrm{d} \eta}{\eta} \mathrm{d} \sigma\right] \\
& -\frac{(\ln \sigma)^{\alpha-1} \Gamma(\alpha-\beta)}{\Delta_{\alpha} \Gamma^{2}(\alpha)}\left[\int_{1}^{2} \int_{1}^{2}(\ln \sigma)^{\alpha-1} \mathrm{y}(\eta) \frac{\mathrm{d} \eta}{\eta} \mathrm{d} \mu(\sigma)-\int_{1}^{2} \int_{1}^{\sigma}\left(\ln \frac{\sigma}{\eta}\right)^{\alpha-1} \mathrm{y}(\eta) \frac{\mathrm{d} \eta}{\eta} \mathrm{d} \mu(\sigma)\right] \\
& -\frac{(\ln \sigma)^{\alpha-1}}{\Gamma(\alpha)} \int_{1}^{2} \mathrm{y}(\eta) \frac{\mathrm{d} \eta}{\eta}+\frac{1}{\Gamma(\alpha)} \int_{1}^{\sigma}\left(\ln \frac{\sigma}{\eta}\right)^{\alpha-1} \mathrm{y}(\eta) \frac{\mathrm{d} \eta}{\eta} \\
& +\frac{(\ln \sigma)^{\alpha-1}}{\Delta_{\alpha} \Gamma(\alpha)}\left[\int_{1}^{\zeta} \int_{1}^{2} \varphi_{\alpha}(\sigma)(\ln \sigma)^{\alpha-1-\beta} \mathcal{U}_{\phi_{1}}(\eta) \frac{\mathrm{d} \eta}{\eta} \mathrm{d} \sigma-\int_{1}^{\zeta} \int_{1}^{\sigma} \varphi_{\alpha}(\sigma)\left(\ln \frac{\sigma}{\eta}\right)^{\alpha-1-\beta} \mathscr{U}_{\phi_{1}}(\eta) \frac{\mathrm{d} \eta}{\eta} \mathrm{d} \sigma\right] \\
& +\frac{(\ln \sigma)^{\alpha-1} \Gamma(\alpha-\beta)}{\Delta_{\alpha} \Gamma^{2}(\alpha)}\left[\int_{1}^{2} \int_{1}^{2}(\ln \sigma)^{\alpha-1} \mathscr{U}_{\phi_{1}}(\eta) \frac{\mathrm{d} \eta}{\eta} \mathrm{d} \mu(\sigma)-\int_{1}^{2} \int_{1}^{\sigma}\left(\ln \frac{\sigma}{\eta}\right)^{\alpha-1} \mathcal{U}_{\phi_{1}}(\eta) \frac{\mathrm{d} \eta}{\eta} \mathrm{d} \mu(\sigma)\right] \\
& +\frac{(\ln \sigma)^{\alpha-1}}{\Gamma(\alpha)} \int_{1}^{2} \mathscr{U}_{\phi_{1}}(\eta) \frac{\mathrm{d} \eta}{\eta}-\frac{1}{\Gamma(\alpha)} \int_{1}^{\sigma}\left(\ln \frac{\sigma}{\eta}\right)^{\alpha-1} \mathscr{U}_{\phi_{1}}(\eta) \frac{\mathrm{d} \eta}{\eta} .
\end{aligned}
$$


For computational convenience, we use $\omega_{1}(\sigma)$ for the sum of terms which are free of $\mathscr{U}_{\phi_{1}}$, so we have

$$
\begin{aligned}
\omega_{1}(\sigma)= & -\frac{(\ln \sigma)^{\alpha-1}}{\Delta_{\alpha} \Gamma(\alpha)}\left[\int_{1}^{\zeta} \int_{1}^{2} \varphi_{\alpha}(\sigma)(\ln \sigma)^{\alpha-1-\beta} \mathrm{y}(\eta) \frac{\mathrm{d} \eta}{\eta} \mathrm{d} \sigma-\int_{1}^{\zeta} \int_{1}^{\sigma} \varphi_{\alpha}(\sigma)\left(\ln \frac{\sigma}{\eta}\right)^{\alpha-1-\beta} \mathrm{y}(\eta) \frac{\mathrm{d} \eta}{\eta} \mathrm{d} \sigma\right] \\
& -\frac{(\ln \sigma)^{\alpha-1} \Gamma(\alpha-\beta)}{\Delta_{\alpha} \Gamma^{2}(\alpha)}\left[\int_{1}^{2} \int_{1}^{2}(\ln \sigma)^{\alpha-1} \mathrm{y}(\eta) \frac{\mathrm{d} \eta}{\eta} \mathrm{d} \mu(\sigma)-\int_{1}^{2} \int_{1}^{\sigma}\left(\ln \frac{\sigma}{\eta}\right)^{\alpha-1} \mathrm{y}(\eta) \frac{\mathrm{d} \eta}{\eta} \mathrm{d} \mu(\sigma)\right] \\
& -\frac{(\ln \sigma)^{\alpha-1}}{\Gamma(\alpha)} \int_{1}^{2} \mathrm{y}(\eta) \frac{\mathrm{d} \eta}{\eta}+\frac{1}{\Gamma(\alpha)} \int_{1}^{\sigma}\left(\ln \frac{\sigma}{\eta}\right)^{\alpha-1} \mathrm{y}(\eta) \frac{\mathrm{d} \eta}{\eta} .
\end{aligned}
$$

So, from the above and taking the absolute value, (158) becomes

$$
\begin{aligned}
\left|\mathrm{u}(\sigma)-\omega_{1}(\sigma)\right| \leqslant & \frac{(\ln \sigma)^{\alpha-1}}{\left|\Delta_{\alpha}\right| \Gamma(\alpha)} \int_{1}^{\zeta} \int_{1}^{2}\left|\varphi_{\alpha}(\sigma)\right|(\ln \sigma)^{\alpha-1-\beta}\left|\mathcal{U}_{\phi_{1}}(\eta)\right| \frac{\mathrm{d} \eta}{\eta} \mathrm{d} \sigma \\
& +\frac{(\ln \sigma)^{\alpha-1}}{\left|\Delta_{\alpha}\right| \Gamma(\alpha)} \int_{1}^{\zeta} \int_{1}^{\sigma}\left|\varphi_{\alpha}(\sigma)\right|\left(\ln \frac{\sigma}{\eta}\right)^{\alpha-1-\beta}|| \mathcal{U}_{\phi_{1}}(\eta) \mid \frac{\mathrm{d} \eta}{\eta} \mathrm{d} \sigma \\
& +\frac{(\ln \sigma)^{\alpha-1} \Gamma(\alpha-\beta)}{\left|\Delta_{\alpha}\right| \Gamma^{2}(\alpha)} \int_{1}^{2} \int_{1}^{2}(\ln \sigma)^{\alpha-1}\left|\mathcal{U}_{\phi_{1}}(\eta)\right| \frac{\mathrm{d} \eta}{\eta} \mathrm{d} \mu(\sigma) \\
& +\frac{(\ln \sigma)^{\alpha-1} \Gamma(\alpha-\beta)}{\left|\Delta_{\alpha}\right| \Gamma^{2}(\alpha)} \int_{1}^{2} \int_{1}^{\sigma}\left|\left(\ln \frac{\sigma}{\eta}\right)^{\alpha-1}\right|\left|\mathcal{U}_{\phi_{1}}(\eta)\right| \frac{\mathrm{d} \eta}{\eta} \mathrm{d} \mu(\sigma) \\
& +\frac{(\ln \sigma)^{\alpha-1}}{\Gamma(\alpha)} \int_{1}^{2}\left|\mathcal{U}_{\phi_{1}}(\eta)\right| \frac{\mathrm{d} \eta}{\eta}+\frac{1}{\Gamma(\alpha)} \int_{1}^{\sigma}\left|\left(\ln \frac{\sigma}{\eta}\right)^{\alpha-1}\right|\left|\mathcal{U}_{\phi_{1}}(\eta)\right| \frac{\mathrm{d} \eta}{\eta} .
\end{aligned}
$$

Using 2 of Lemma 6 and 1 of Remark 5, we get

$$
\left|\mathrm{u}(\sigma)-\omega_{1}(\sigma)\right| \leqslant \mathscr{Y}_{\alpha} \epsilon_{\alpha} .
$$

In the same way, we have

$$
\left|\mathrm{v}(\sigma)-\omega_{2}(\sigma)\right| \leqslant \mathscr{Y}_{\gamma} \epsilon_{\gamma} .
$$

holds, then the given system (7) is stable in the sense of $\mathscr{H} \mathscr{U}$.

Proof. Let $(\mathrm{u}, \mathrm{v}) \in \mathbb{X} \times \mathbb{X}$ be the solution of (149) and $(\widehat{\mathrm{u}}, \widehat{\mathrm{v}}) \in \mathbb{X} \times \mathbb{X}$ be the solution to the system

Theorem 13. Under the hypothesis $\left(\mathbf{M}_{10}\right)$ and if

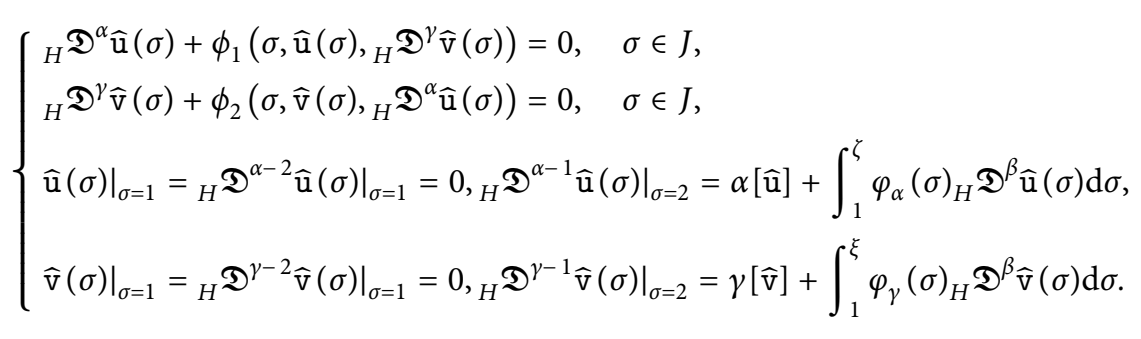


Then, for $\sigma \in J$, ( $\widehat{\mathrm{u}}, \widehat{\mathrm{v}})$ is the solution of (164), i.e.,

Consider

$\left\{\begin{array}{l}\widehat{\mathrm{u}}(\sigma)=\int_{1}^{2} \mathbb{G}_{\alpha}(\sigma, \eta) \phi_{1}\left(\eta, \widehat{\mathrm{u}}(\eta),{ }_{H} \mathfrak{D}^{\gamma} \widehat{\mathrm{v}}(\eta)\right) \frac{\mathrm{d} \eta}{\eta}, \\ \widehat{\mathrm{v}}(\sigma)=\int_{1}^{2} \mathbb{G}_{\gamma}(\sigma, \eta) \phi_{2}\left(\eta, \widehat{\mathrm{v}}(\eta), H^{2} \mathfrak{D}^{\alpha} \widehat{\mathrm{u}}(\eta)\right) \frac{\mathrm{d} \eta}{\eta} .\end{array}\right.$

$$
\begin{aligned}
|\mathrm{u}(\sigma)-\widehat{\mathrm{u}}(\sigma)| \leqslant & \left|\mathrm{u}(\sigma)-\omega_{1}(\sigma)\right|+\left|\omega_{1}(\sigma)-\widehat{\mathrm{u}}(\sigma)\right| \\
\leqslant & \mathscr{Y}_{\alpha} \epsilon_{\alpha}+\frac{(\ln \sigma)^{\alpha-1}}{\left|\Delta_{\alpha}\right| \Gamma(\alpha)} \int_{1}^{\zeta} \int_{1}^{2}\left|\varphi_{\alpha}(\sigma)\right|(\ln \sigma)^{\alpha-1-\beta}\left|\mathrm{y}(\eta)-\mathrm{y}_{\widehat{\mathrm{u}}}(\eta)\right| \frac{\mathrm{d} \eta}{\eta} \mathrm{d} \sigma \\
& +\frac{(\ln \sigma)^{\alpha-1}}{\left|\Delta_{\alpha}\right| \Gamma(\alpha)} \int_{1}^{\zeta} \int_{1}^{\sigma}\left|\varphi_{\alpha}(\sigma)\right|\left(\ln \frac{\sigma}{\eta}\right)^{\alpha-1-\beta}|| \mathrm{y}(\eta)-\mathrm{y}_{\widehat{\mathrm{u}}}(\eta) \mid \frac{\mathrm{d} \eta}{\eta} \mathrm{d} \sigma \\
& +\frac{(\ln \sigma)^{\alpha-1} \Gamma(\alpha-\beta)}{\left|\Delta_{\alpha}\right| \Gamma^{2}(\alpha)} \int_{1}^{2} \int_{1}^{2}(\ln \sigma)^{\alpha-1}\left|\mathrm{y}(\eta)-\mathrm{y}_{\widehat{\mathrm{u}}}(\eta)\right| \frac{\mathrm{d} \eta}{\eta} \mathrm{d} \mu(\sigma) \\
& +\frac{(\ln \sigma)^{\alpha-1} \Gamma(\alpha-\beta)}{\left|\Delta_{\alpha}\right| \Gamma^{2}(\alpha)} \int_{1}^{2} \int_{1}^{\sigma}\left|\left(\ln \frac{\sigma}{\eta}\right)^{\alpha-1}\right|\left|\mathrm{y}(\eta)-\mathrm{y}_{\widehat{\mathrm{u}}}(\eta)\right| \frac{\mathrm{d} \eta}{\eta} \mathrm{d} \mu(\sigma) \\
& +\frac{(\ln \sigma)^{\alpha-1}}{\Gamma(\alpha)} \int_{1}^{2}\left|\mathrm{y}(\eta)-\mathrm{y}_{\widehat{\mathrm{u}}}(\eta)\right| \frac{\mathrm{d} \eta}{\eta}+\frac{1}{\Gamma(\alpha)} \int_{1}^{\sigma}\left|\left(\ln \frac{\sigma}{\eta}\right)^{\alpha-1}\right|\left|\mathrm{y}(\eta)-\mathrm{y}_{\widehat{\mathrm{u}}}(\eta)\right| \frac{\mathrm{d} \eta}{\eta},
\end{aligned}
$$

where $\mathrm{y}, \mathrm{y}_{\widehat{u}} \in \mathbb{X}$ are of the form

$$
\begin{gathered}
\mathrm{y}(\sigma)=-\phi_{1}(\sigma, \mathrm{u}(\sigma) \mathrm{z}(\sigma)), \\
\mathrm{y}_{\widehat{\mathrm{u}}}(\sigma)=-\phi_{1}\left(\sigma, \widehat{\mathrm{u}}(\sigma), \mathrm{z}_{\widehat{\mathrm{v}}}(\sigma)\right) .
\end{gathered}
$$

By $\left(\mathbf{M}_{10}\right)$, we get

$$
\begin{aligned}
\left|\mathrm{y}(\sigma)-\mathrm{y}_{\widehat{\mathrm{u}}}(\sigma)\right| & =\left|-\left(\phi_{1}(\sigma, \mathrm{u}(\sigma), \mathbf{z}(\sigma))-\phi_{1}\left(\sigma, \widehat{\mathrm{u}}(\sigma), \mathbf{z}_{\widehat{\mathrm{v}}}(\sigma)\right)\right)\right| \\
& \leqslant \mathfrak{Q}_{\phi_{1}}|\mathrm{u}(\sigma)-\widehat{\mathrm{u}}(\sigma)|+\mathfrak{Q}_{\phi_{1}}^{*}\left|\mathbf{z}(\sigma)-\mathbf{z}_{\widehat{\mathrm{v}}}(\sigma)\right|,
\end{aligned}
$$

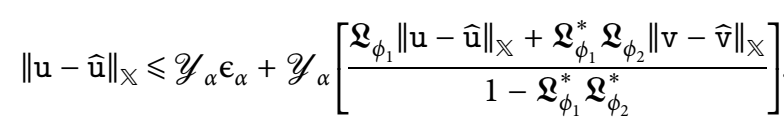

Similarly, we have

and we obtain

$$
\|\mathrm{v}-\widehat{\mathrm{v}}\|_{\mathbb{X}} \leqslant \mathscr{Y}_{\gamma} \epsilon_{\gamma}+\mathscr{Y}_{\gamma}\left[\frac{\mathfrak{Q}_{\phi_{1}} \mathfrak{Q}_{\phi_{2}}^{*}\|\mathrm{u}-\widehat{\mathrm{u}}\|_{\mathbb{X}}+\mathfrak{\Omega}_{\phi_{2}}\|\mathrm{v}-\widehat{\mathrm{v}}\|_{\mathbb{X}}}{1-\mathfrak{\Omega}_{\phi_{1}}^{*} \mathfrak{Q}_{\phi_{2}}^{*}}\right],
$$

where $z, z_{\widehat{v}} \in \mathbb{X}$ in the form

$$
\left|\mathrm{y}(\sigma)-\mathrm{y}_{\widehat{u}}(\sigma)\right| \leqslant \frac{\mathfrak{Q}_{\phi_{1}}|\mathrm{u}(\sigma)-\widehat{\mathrm{u}}(\sigma)|+{\mathfrak{\mathfrak { Q } _ { 1 }}}_{\phi_{1}}^{*} \mathfrak{Q}_{\phi_{2}}|\mathrm{v}(\sigma)-\widehat{\mathrm{v}}(\sigma)|}{1-\mathfrak{Q}_{\phi_{1}}^{*} \mathfrak{Q}_{\phi_{2}}^{*}} .
$$

$$
\begin{aligned}
\mathrm{z}(\sigma) & =-\phi_{2}(\sigma, \mathrm{v}(\sigma), \mathrm{y}(\sigma)), \\
\mathrm{z}_{\widehat{\mathrm{v}}}(\sigma) & =-\phi_{2}\left(\sigma, \widehat{\mathrm{v}}(\sigma), \mathrm{y}_{\widehat{\mathrm{u}}}(\sigma)\right) .
\end{aligned}
$$

We write (170) and (171) as

Using 2 of Lemma 6 and (168) in (166), we get

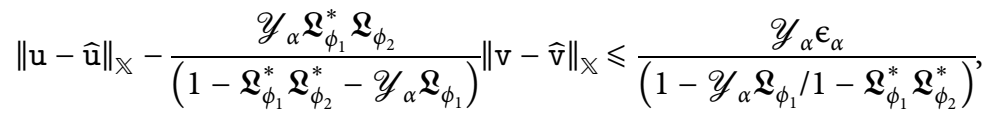

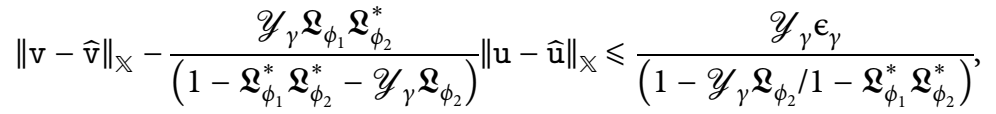


respectively. Let

$$
\begin{aligned}
& \mathscr{K}_{1}=\frac{\mathscr{Y}_{\alpha} \mathfrak{Q}_{\phi_{1}}^{*} \mathfrak{Q}_{\phi_{2}}}{\left(1-\mathfrak{\Omega}_{\phi_{1}}^{*} \mathfrak{\Omega}_{\phi_{2}}^{*}-\mathscr{Y}_{\alpha} \mathfrak{\Omega}_{\phi_{1}}\right)}, \mathscr{K}_{2}=\frac{\mathscr{Y}_{\alpha}}{\left(1-\mathscr{Y}_{\alpha} \mathfrak{Q}_{\phi_{1}} / 1-\mathfrak{\Omega}_{\phi_{1}}^{*} \mathfrak{\Omega}_{\phi_{2}}^{*}\right)},
\end{aligned}
$$

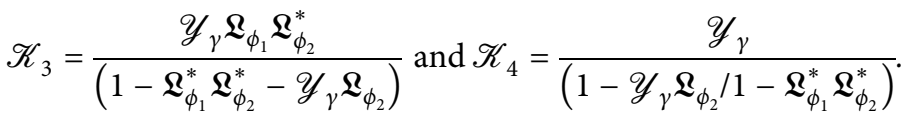

In the matrix form, the abovementioned inequalities can be written as

$$
\left[\begin{array}{cc}
1 & -\mathscr{K}_{1} \\
-\mathscr{K}_{3} & 1
\end{array}\right]\left[\begin{array}{l}
\|\mathrm{u}-\widehat{\mathrm{u}}\|_{\mathbb{X}} \\
\|\mathrm{v}-\widehat{\mathrm{v}}\|_{\mathbb{X}}
\end{array}\right] \leqslant\left[\begin{array}{c}
\mathscr{K}_{2} \epsilon_{\alpha} \\
\mathscr{K}_{4} \epsilon_{\gamma}
\end{array}\right] .
$$

Solving the abovementioned inequality, we have

$$
\left[\begin{array}{c}
\|\mathrm{u}-\widehat{\mathrm{u}}\|_{\mathbb{X}} \\
\|\mathrm{v}-\widehat{\mathrm{v}}\|_{\mathbb{X}}
\end{array}\right] \leqslant\left[\begin{array}{c}
\frac{1}{1-\mathscr{K}_{1} \mathscr{K}_{3}} \frac{\mathscr{K}_{1}}{1-\mathscr{K}_{1} \mathscr{K}_{3}} \\
\frac{\mathscr{K}_{3}}{1-\mathscr{K}_{1} \mathscr{K}_{3}} \frac{1}{1-\mathscr{K}_{1} \mathscr{K}_{3}}
\end{array}\right]\left[\begin{array}{l}
\mathscr{K}_{2} \epsilon_{\alpha} \\
\mathscr{K}_{4} \epsilon_{\gamma}
\end{array}\right] .
$$

Further simplification gives

$$
\begin{aligned}
\|\mathrm{u}-\widehat{\mathrm{u}}\|_{\mathbb{X}} & =\frac{\mathscr{K}_{2} \epsilon_{\alpha}}{1-\mathscr{K}_{1} \mathscr{K}_{3}}+\frac{\mathscr{K}_{1} \mathscr{K}_{4} \epsilon_{\gamma}}{1-\mathscr{K}_{1} \mathscr{K}_{3}}, \\
\|\mathrm{v}-\widehat{\mathrm{v}}\|_{\mathbb{X}} & =\frac{\mathscr{K}_{4} \epsilon_{\gamma}}{1-\mathscr{K}_{1} \mathscr{K}_{3}}+\frac{\mathscr{K}_{2} \mathscr{K}_{3} \epsilon_{\alpha}}{1-\mathscr{K}_{1} \mathscr{K}_{3}},
\end{aligned}
$$

from which we have

$$
\begin{aligned}
\|\mathrm{u}-\widehat{\mathrm{u}}\|_{\mathbb{X}}+\|\mathrm{v}-\widehat{\mathrm{v}}\|_{\mathbb{X}}= & \frac{\mathscr{K}_{2} \epsilon_{\alpha}}{1-\mathscr{K}_{1} \mathscr{K}_{3}}+\frac{\mathscr{K}_{4} \epsilon_{\gamma}}{1-\mathscr{K}_{1} \mathscr{K}_{3}} \\
& +\frac{\mathscr{K}_{1} \mathscr{K}_{4} \epsilon_{\gamma}}{1-\mathscr{K}_{1} \mathscr{K}_{3}}+\frac{\mathscr{K}_{2} \mathscr{K}_{3} \epsilon_{\alpha}}{1-\mathscr{K}_{1} \mathscr{K}_{3}} .
\end{aligned}
$$

Let $\varepsilon_{\alpha, \gamma}=\max \left\{\varepsilon_{\alpha}, \varepsilon_{\gamma}\right\}$; then, from (178), we have

$$
\|(\mathrm{u}, \mathrm{v})-(\widehat{\mathrm{u}}, \widehat{\mathrm{v}})\|_{\mathbb{X} \times \mathbb{X}} \leqslant \mathfrak{G}_{\alpha, \gamma} \epsilon_{\alpha, \gamma},
$$

where

$$
\mathfrak{夭}_{\alpha, \gamma}=\frac{\mathscr{K}_{2}}{1-\mathscr{K}_{1} \mathscr{K}_{3}}+\frac{\mathscr{K}_{4}}{1-\mathscr{K}_{1} \mathscr{K}_{3}}+\frac{\mathscr{K}_{1} \mathscr{K}_{4}}{1-\mathscr{K}_{1} \mathscr{K}_{3}}+\frac{\mathscr{K}_{2} \mathscr{K}_{3}}{1-\mathscr{K}_{1} \mathscr{K}_{3}} .
$$

Remark 6. By setting $\mathfrak{S}^{\prime}\left(\epsilon_{\alpha, \gamma}\right)=\mathfrak{c}_{\alpha, \gamma} \epsilon_{\alpha, \gamma}, \mathfrak{c}^{\prime}(0)=0$ in (179), by Definition 8 , the given system (7) is $\mathscr{G} \mathscr{H} \mathscr{U} \mathcal{S}$.

Remark 7. Under the hypothesis $\left(\mathbf{M}_{12}\right)$ and (163) and by using Definitions 9 and 10, one can repeat the process of Lemma 9 and the Theorem 13, and the system (7) will be $\mathscr{H} \mathscr{U} \mathscr{R}$ and $\mathscr{G} \mathscr{H} \mathscr{R} \mathcal{S}$.

\section{Examples}

In this section, we present three examples to demonstrate the existence and stability of the obtained results.

\section{Example 1.}

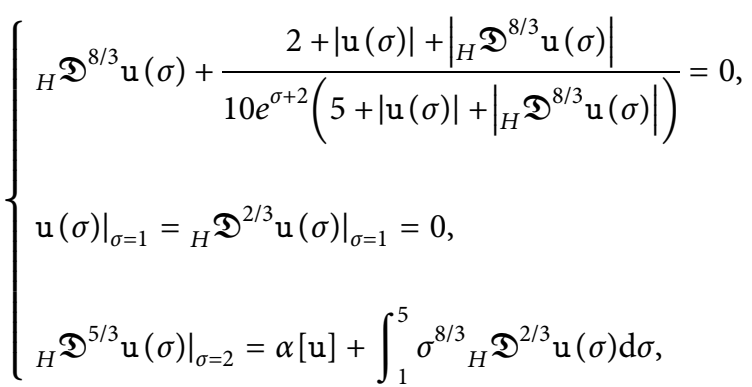

where $\quad \alpha=8 / 3, \beta=2 / 3, \zeta=5, \mathfrak{P}=20, \mathfrak{F}=20, \rho=5 / 3, \varphi$ $(\sigma)=\sigma^{8 /} 3 \geqslant 1$, for $\sigma \in[1,2]$. Moreover, $\alpha[\mathrm{u}]=\mathrm{u}(1 / 5) / 25$. Set

$\phi(\sigma, \mathrm{y}, \mathrm{z})=\frac{2+|\mathrm{y}|+|\mathrm{z}|}{10 e^{\sigma+2}(5+|\mathrm{y}|+|\mathrm{z}|)}, \quad \sigma \in[1,2], \mathrm{y}, \mathrm{z} \in \mathbb{R}$.

Now, for any $\mathrm{y}, \mathrm{z}, \widehat{\mathrm{y}}, \widehat{\mathrm{z}} \in \mathbb{R}$ and $\sigma \in[1,2]$, we have

$$
|\phi(\sigma, \mathrm{y}, \mathrm{z})-\phi(\sigma, \widehat{\mathrm{y}}, \widehat{\mathrm{z}})| \leqslant \frac{1}{10 e^{2}}|\mathrm{y}-\widehat{\mathrm{y}}|+\frac{1}{10 e^{2}}|\mathrm{z}-\widehat{\mathrm{z}}| \text {. }
$$

Hence, $\left(\mathbf{M}_{4}\right)$ is satisfied with $\mathfrak{Q}_{\phi}=\mathbf{Q}_{\phi}^{*}=1 / 10 e^{2}$. Also, for any $y, z \in \mathbb{R}$, we have

$$
|\phi(\sigma, \mathrm{y}, \mathrm{z})| \leqslant \frac{1}{10 e^{\sigma+2}}(2+|\mathrm{y}|+|\mathrm{z}|) .
$$

Hence, $\left(\mathbf{M}_{3}\right)$ is satisfied with

$$
\pi_{1}(\varrho)=\frac{1}{5 e^{\sigma+2}}, \pi_{2}(\varrho)=\pi_{3}(\varrho)=\frac{1}{10 e^{\sigma+2}},
$$

where $\pi_{1}^{*}=1 / 5 e^{2}$ and $\pi_{2}^{*}=\pi_{3}^{*}=1 / 10 e^{2}$.

From Theorem 7 , we use the inequality which are found as

$$
\frac{\mathfrak{Q}_{\phi}}{1-\mathfrak{Q}_{\phi}^{*}} \mathscr{y} \approx 0.0134252<1
$$

Hence, (181) has a unique solution. 
Furthermore, $\mathscr{K}>0$ with condition (126) holds, and

$$
\frac{\mathfrak{R}_{\phi}}{1-\mathfrak{Q}_{\phi}^{*}} \mathscr{y} \approx 0.0134252<1 .
$$

Hence, with the help of Theorem 11, the given system (181) is $\mathscr{H} \mathscr{U} \mathcal{S}$ and, hence, $\mathscr{G} \mathscr{H} \mathscr{U} \mathcal{S}$. Also, by checking the conditions of Theorem 12, it can be easily verified that the considered problem (181) is $\mathscr{H} \mathscr{U} \mathscr{R} \mathcal{S}$ and $\mathscr{G} \mathscr{H} \mathscr{U} \mathscr{S}$.

\section{Example 2.}

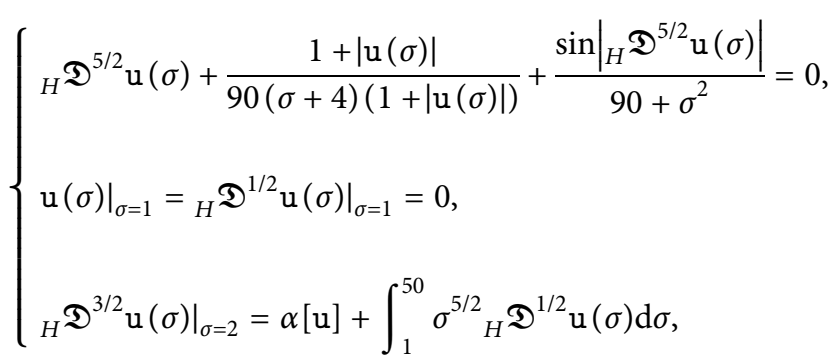

where $\quad \alpha=5 / 2, \beta=1 / 2, \zeta=50, \mathfrak{P}=50, \mathfrak{F}=50, \rho=3 / 2$, $\varphi(\sigma)=\sigma^{5 /} 2 \geqslant 0$, for $\sigma \in[1,2]$. Moreover, $\alpha[\mathrm{u}]=\mathrm{u}(1 / 5) / 15$.

Set

$$
\phi(\sigma, \mathrm{u}(\sigma), \mathrm{z}(\sigma))=\frac{1+|\mathrm{u}(\sigma)|}{90(\sigma+4)(1+|\mathrm{u}(\sigma)|)}+\frac{\sin \left|{ }_{H} \mathfrak{D}^{5 / 2} \mathrm{u}(\sigma)\right|}{90+\sigma^{2}}
$$

Now, for any $y, z, \widehat{y}, \widehat{z} \in \mathbb{R}$ and $\sigma \in[1,2]$, we have

$$
|\phi(\sigma, y, z)-\phi(\sigma, y, \widehat{z})| \leq \frac{1}{90}|y-\widehat{y}|+\frac{1}{90}|z-\widehat{z}| .
$$

Hence, $\left(\mathbf{M}_{4}\right)$ is satisfied with $\mathfrak{\Omega}_{\phi}=\mathfrak{\Omega}_{\phi}^{*}=1 / 90$.

Also, for any $y, z \in \mathbb{R}$, we have

$$
|\phi(\sigma, y, z)| \leq \frac{1}{90(\sigma+4)}(1+|y|)+\frac{1}{90+\sigma^{2}}|z| .
$$

Hence, $\left(\mathbf{M}_{3}\right)$ is satisfied with

$$
\begin{aligned}
& \pi_{1}(\sigma)=\pi_{2}(\sigma)=\frac{1}{90(\sigma+4)}, \\
& \pi_{3}(\sigma)=\frac{1}{90+\sigma^{2}},
\end{aligned}
$$

where $\pi_{1}^{*}=\pi_{2}^{*}=\pi_{3}^{*}=1 / 90$.

From Theorem 7 , we use the inequality which are found as

$$
\frac{\mathfrak{R}_{\phi}}{1-\mathfrak{Q}_{\phi}^{*}} \mathscr{y} \approx 0.0160445<1
$$

Hence, (188) has a unique solution.

Furthermore, $\mathscr{K}>0$ with condition (126) holds, and

$$
\frac{\mathfrak{R}_{\phi}}{1-\mathfrak{\Omega}_{\phi}^{*}} \mathscr{Y} \approx 0.0160445<1 .
$$

Hence, with the help of Theorem 11, the given system (188) is $\mathscr{H} \mathscr{U} \mathcal{S}$ and, hence, $\mathscr{G} \mathscr{H} \mathscr{U} \mathcal{S}$. Also, by checking the conditions of Theorem 11, we can find that the considered problem (188) is $\mathscr{H} \mathscr{U} \mathscr{R} \mathcal{S}$ and $\mathscr{G} \mathscr{H} \mathscr{U} \mathscr{S}$.

Example 3.

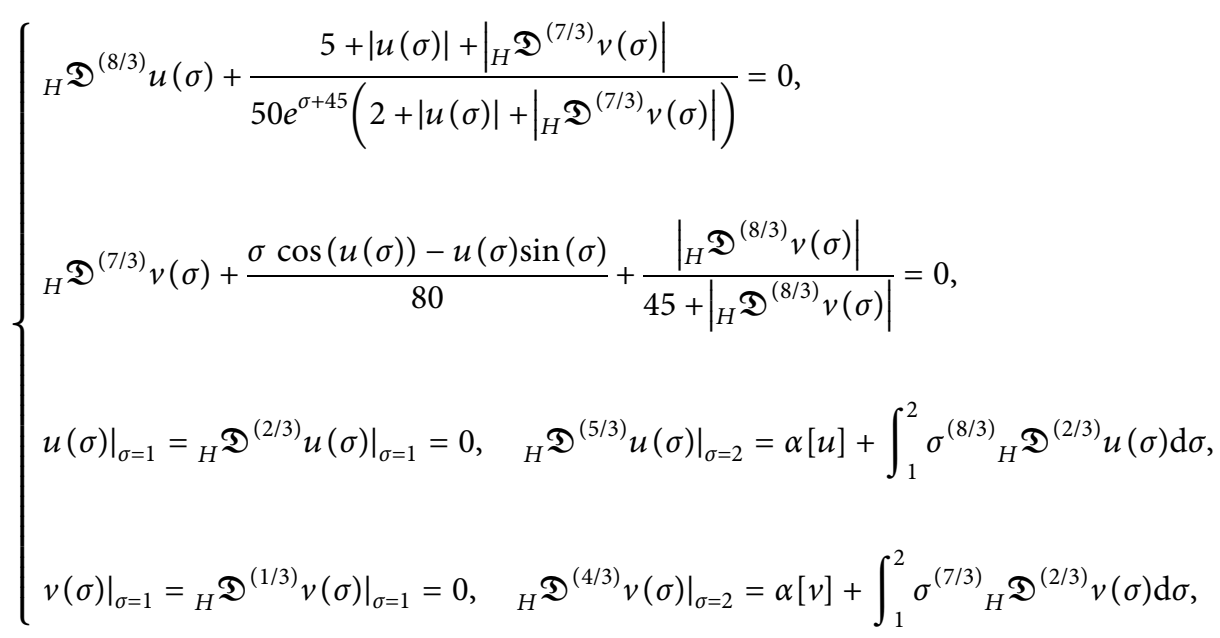


where $\alpha=8 / 3, \beta=2 / 3, \gamma=7 / 3, \delta=1 / 3, \zeta=\xi=2, \mathfrak{P}=5$, $\mathfrak{E}_{\alpha}=\mathfrak{E}_{\gamma}=1, \quad \rho=3 / 2, \varphi_{\alpha}(\sigma)=\sigma^{8 / 3} \geqslant 0, \varphi_{\gamma}(\sigma)=\sigma^{7 / 3} \geqslant 0$, for $\sigma \in[1,2]$. Moreover, $\alpha[\mathrm{u}]=\alpha[\mathrm{v}]=\mathrm{u}(1 / 5) / 25$.

$$
\begin{aligned}
& \phi_{1}\left(\sigma, u(\sigma),{ }_{H} \mathfrak{D}^{\gamma} v(\sigma)\right)=\frac{5+|u(\sigma)|+\left|{ }_{H} \mathfrak{D}^{(7 / 3)} v(\sigma)\right|}{50 e^{\sigma+45}\left(2+|u(\sigma)|+\left.\right|_{H} \mathfrak{D}^{(7 / 3)} v(\sigma) \mid\right)}, \\
& \phi_{2}\left(\sigma, v(\sigma),{ }_{H} \mathfrak{D}^{\alpha} u(\sigma)\right)=\frac{\sigma \cos (u(\sigma))-u(\sigma) \sin (\sigma)}{80}+\frac{\left|\mathfrak{D}^{(8 / 3)} v(\sigma)\right|}{45+\left|{ }_{H} \mathfrak{D}^{(8 / 3)} v(\sigma)\right|} .
\end{aligned}
$$

By simple computations, we found that

$$
\mathfrak{Q}_{\phi_{1}}=\mathbf{Q}_{\phi_{1}}^{*}=\frac{1}{50 e^{45}}, \mathfrak{Q}_{\phi_{2}}=\mathbf{Q}_{\phi_{2}}^{*}=\frac{1}{45} .
$$

From Theorem 9, we use the inequality which are found as

$$
\frac{\mathscr{Y}_{\alpha}\left(\mathfrak{Q}_{\phi_{1}}+\mathfrak{R}_{\phi_{1}}^{*} \mathfrak{Q}_{\phi_{2}}\right)+\mathscr{Y}_{\gamma}\left(\mathfrak{Q}_{\phi_{1}} \mathfrak{R}_{\phi_{2}}^{*}+\mathfrak{Q}_{\phi_{2}}\right)}{1-\mathfrak{Q}_{\phi_{1}}^{*} \mathfrak{Q}_{\phi_{2}}^{*}} \approx 0.0254987<1
$$

Hence, (195) has a unique solution, and

$$
1-\mathscr{K}_{1} \mathscr{K}_{3} \approx 1>0 .
$$

Hence. with the help of Theorem 13, the given system (195) is $\mathscr{H} \mathscr{U} \mathcal{S}$. Also, by using $\left(\mathbf{M}_{12}\right)$ and Remark 6 and 7 , the given system is $\mathscr{G} \mathscr{H} \mathcal{U} \mathcal{S}, \mathscr{H} \mathscr{U} \mathscr{S}$, and $\mathscr{G} \mathscr{H} \mathscr{R} \mathcal{S}$.

\section{Conclusions}

We have obtained some appropriate conditions for the existence, uniqueness, and Ulam's stabilities of the system (5) and its corresponding coupled system (7). The required results are obtained using the Banach contraction principle, Schaefer's fixed point theorem, Arzela-Ascoli theorem, and Leray-Schauder of the cone type. Also, with the help of some sufficient conditions, we have derived different kinds of Ulam's stabilities for the solution of the problem (5) and the coupled problem (7). Additionally, examples are given to support the main results.

\section{Data Availability}

No data were used to support this work.

\section{Conflicts of Interest}

The authors declare that they have no conflicts of interest.

\section{Authors' Contributions}

The authors contributed equally and significantly in writing this paper. All authors read and approved the final manuscript.

\section{Acknowledgments}

This work was jointly supported by the National Natural Science Foundation of China (11661016), Training Object of High Level and Innovative Talents of Guizhou Province [(2016)4006], and Major Research Project of Innovative Group in Guizhou Education Department [(2018)012].

\section{References}

[1] G. W. Leibniz, Mathematica Shiften, Georg Olms Verlagsbuch-Handlung, Hildesheim, Germany, 1962.

[2] J. Sabatier, O. P. Agrawal, and J. A. T. Machado, Advances in Fractional Calculus, Springer, Dordrecht, Netherlands, 2007.

[3] F. A. Rihan, "Numerical modeling of fractional order biological systems," Abstract and Applied Analysis, vol. 2013, Article ID 816803, 11 pages, 2013.

[4] B. M. Vintagre, I. Podlybni, A. Hernandez, and V. Feliu, "Some approximations of fractional order operators used in control theory and applications," Fractional Calculus and Applied Analysis, vol. 3, no. 3, pp. 231-248, 2000.

[5] N. Ahmad, Z. Ali, K. Shah, A. Zada, and G. Rahman, "Analysis of implicit type nonlinear dynamical problem of impulsive fractional differentail equations," Complexity, vol. 2018, Article ID 6423974, 15 pages, 2018.

[6] R. Rizwan, A. Zada, and X. Wang, "Stability analysis of non linear implicit fractional Langevin equation with noninstantaneous impulses," Advances in Difference Equations, vol. 2019, p. 85, 2019.

[7] J. Hadamard, Essai sur l'étude des fonctions, données par leur développement de Taylor, Gauthier-Villars, Paris, France, 1892.

[8] M. Benchohra, J. E. Lazreg, and J. E. Lazreg, "Existence and ulam stability for nonlinear implicit fractional differential equations with hadamard derivative," Studia Universitatis Babes-Bolyai Matematica, vol. 62, no. 1, pp. 27-38, 2017.

[9] J. Jiang, D. O’Regan, J. Xu, and Z. Fu, "Positive solutions for a system of nonlinear hadamard fractional differential equations involving coupled integral boundary conditions," Journal of Inequalities and Applications, vol. 2019, no. 1, pp. 1-18, 2019.

[10] U. Riaz, A. Zada, Z. Ali, M. Ahmad, J. Xu, and Z. Fu, "Analysis of nonlinear coupled systems of impulsive fractional differential equations with Hadamard derivatives," Mathematical Problems in Engineering, vol. 2019, Article ID 5093572, 20 pages, 2019. 
[11] G. Wang, K. Pei, and Y. Chen, "Stability analysis of nonlinear Hadamard fractional differential system," Journal of the Franklin Institute, vol. 356, no. 12, pp. 6538-6546, 2019.

[12] Z. Ali, A. Zada, and K. Shah, "Ulam stability results for the solutions of nonlinear implicit fractional order differential equations," Hacettepe Journal of Mathematics and Statistics, vol. 48, no. 4, pp. 1092-1109, 2018.

[13] R. Rizwan and A. Zada, "Nonlinear impulsive Langevin equation with mixed derivatives," Mathematical Methods in the Applied Sciences, vol. 43, no. 1, pp. 427-442, 2020.

[14] A. Zada, M. Alam, and U. Riaz, "Analysis of q-fractional implicit boundary value problems having stieltjes integral conditions," Mathematical Methods in the Applied Sciences, vol. 44, no. 6, pp. 4381-4413, 2020.

[15] Z. Ali, A. Zada, and K. Shah, "On ulam's stability for a coupled systems of nonlinear implicit fractional differential equations," Bulletin of the Malaysian Mathematical Sciences Society, vol. 42, no. 5, pp. 2681-2699, 2019.

[16] Z. Ali, A. Zada, and K. Shah, "Ulam stability to a toppled systems of nonlinear implicit fractional order boundary value problem," Boundary Value Problems, vol. 175, 2018.

[17] V. D. Gejji, "Positive solutions of a system of non-autonomous fractional differential equations," Journal of Mathematical Analysis and Applications, vol. 302, pp. 56-64, 2005.

[18] M. P. Lazarević, "Finite time stability analysis of PD $\alpha$ fractional control of robotic time-delay systems," Mechanics Research Communications, vol. 33, pp. 269-279, 2006.

[19] S. M. Ulam, A Collection of the Mathematical Problems, Interscience, New York, NY, USA, 1960.

[20] D. H. Hyers, G. Isac, and T. M. Rassias, Stability of Functional Equations in Several Variables, Birkh"auser, Boston, MA, USA, 1998.

[21] S.-M. Jung, "Hyers-Ulam stability of linear differential equations of first order, II," Applied Mathematics Letters, vol. 19, no. 9, pp. 854-858, 2006.

[22] T. Li and A. Zada, "Connections between Hyers-Ulam stability and uniform exponential stability of discrete evolution families of bounded linear operators over Banach spaces," Advances in Difference Equations, vol. 2016, no. 1, p. 153, 2016.

[23] M. Obloza, "Hyers stability of the linear differential equation," Rocznik NaukDydakt, Prace Mat.vol. 13, pp. 259-270, 1993.

[24] T. M. Rassias, "On the stability of functional equations and a problem of Ulam," Acta Applicandae Mathematicae, vol. 62, no. 1, pp. 23-130, 2000.

[25] M. Ahmad, A. Zada, and J. Alzabut, "Hyers-Ulam stability of a coupled system of fractional differential equations of HilferHadamard type," Demonstratio Mathematica, vol. 52, no. 1, pp. 283-295, 2019.

[26] M. Ahmad, A. Zada, and X. Wang, "Existence, uniqueness and stability of implicit switched coupled fractional differential equations of $\psi \$ \backslash$ boldsymbol $\{\backslash \mathrm{psi}\}$ \$-hilfer type $\Psi$-hilfer type," International Journal of Nonlinear Sciences and $\mathrm{Nu}$ merical Simulation, vol. 21, no. 3-4, p. 327, 2020.

[27] J. Wang, A. Zada, and H. Waheed, "Stability analysis of a coupled system of nonlinear implicit fractional anti-periodic boundary value problem," Mathematical Methods in the Applied Sciences, vol. 42, no. 18, pp. 6706-6732, 2019.

[28] A. Zada, S. Ali, and T. Li, "Analysis of a new class of impulsive implicit sequential fractional differential equations," International Journal of Nonlinear Sciences and Numerical Simulation, vol. 21, no. 6, p. 571, 2020.

[29] A. Zada, B. Pervaiz, S. O. Shah, and J. Xu, "Stability analysis of first-order impulsive nonautonomous system on timescales,"
Mathematical Methods in the Applied Sciences, vol. 43, no. 8, pp. 5097-5113, 2020.

[30] J. Ren and C. Zhai, "Nonlocal q-fractional boundary value problem with Stieltjes integral conditions," Nonlinear Analysis: Modelling and Control, vol. 24, no. 4, pp. 582-602, 2019.

[31] U. Riaz, A. Zada, Z. Ali, Y. Cui, and J. Xu, "Analysis of coupled systems of implicit impulsive fractional differential equations involving Hadamard derivatives," Advances in Difference Equations, vol. 2019, no. 226, pp. 1-27, 2019.

[32] J. K. Hale and S. M. V. Lunel, Introduction to Functional Differential Equations, p. 99, Springer-Verlag, New York, NY, USA, 1993.

[33] A. Granas and J. Dugundji, Fixed Point Theory, SpringerVerlag, New York, NY, USA, 2003.

[34] V. Gafiychuk, B. Datsko, V. Meleshko, and D. Blackmore, "Analysis of the solutions of coupled nonlinear fractional reaction-diffusion equations," Chaos, Solitons \& Fractals, vol. 41, no. 3, pp. 1095-1104, 2009.

[35] R. L. Wheeden, Measure and Integral: An Introduction to Real Analysis, p. 308, CRC Press, Boco Raton, FL, USA, 2015.

[36] A. Browder, Mathematical Analysis: An Introduction, Springer-Verlag, New York, NY, USA, 1996.

[37] J. Wang, A. Zada, and W. Ali, “Ulam's-type stability of firstorder impulsive differential equations with variable delay in quasi-banach spaces," International Journal of Nonlinear Sciences and Numerical Simulation, vol. 19, no. 5, pp. 553-560, 2018.

[38] I. A. Rus, "Ulam stabilities of ordinary differential equations in a Banach space," Carpathian Journal of Mathematics, vol. 26, pp. 103-107, 2010. 